\title{
Chemical Diversity and Bioactivities of Monoterpene Indole Alkaloids (MIAs) from Six Apocynaceae Genera
}

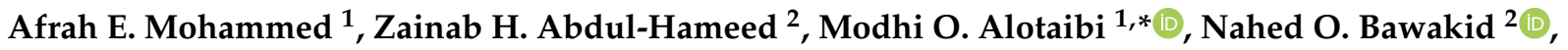 \\ Tariq R. Sobahi ${ }^{2}$, Ahmed Abdel-Lateff ${ }^{3,4}$ (1) and Walied M. Alarif ${ }^{5, *}$ \\ 1 Department of Biology, Faculty of Science, Princess Nourah Bint Abdulrahman University, P.O. Box 84428, \\ Riyadh 11671, Saudi Arabia; AFAMohammed@pnu.edu.sa \\ 2 Department of Chemistry, Faculty of Science, King Abdulaziz University, P.O. Box 80203, Jeddah 21589, \\ Saudi Arabia; flower9@hotmail.com (Z.H.A.-H.); nbawaked@kau.edu.sa (N.O.B.); \\ drtariq_s@hotmail.com (T.R.S.) \\ 3 Department of Natural Products and Alternative Medicine, Faculty of Pharmacy, King Abdulaziz University, \\ P.O. Box 80260, Jeddah 21589, Saudi Arabia; ahmedabdellateff@gmail.com \\ 4 Department of Pharmacognosy, Faculty of Pharmacy, Minia University, Minia 61519, Egypt \\ 5 Department of Marine Chemistry, Faculty of Marine Sciences, King Abdulaziz University, P.O. Box 80207, \\ Jeddah 21589, Saudi Arabia \\ * Correspondence: mouotaebe@pnu.edu.sa (M.O.A.); welaref@kau.edu.sa (W.M.A.)
}

Citation: Mohammed, A.E.

Abdul-Hameed, Z.H.; Alotaibi, M.O.; Bawakid, N.O.; Sobahi, T.R.; Abdel-Lateff, A.; Alarif, W.M. Chemical Diversity and Bioactivities of Monoterpene Indole Alkaloids (MIAs) from Six Apocynaceae Genera. Molecules 2021, 26, 488. https:// doi.org/10.3390/molecules26020488

Academic Editor: John C. D'Auria

Received: 20 December 2020

Accepted: 11 January 2021

Published: 18 January 2021

Publisher's Note: MDPI stays neutral with regard to jurisdictional claims in published maps and institutional affiliations.

Copyright: (c) 2021 by the authors. Licensee MDPI, Basel, Switzerland. This article is an open access article distributed under the terms and conditions of the Creative Commons Attribution (CC BY) license (https:// creativecommons.org/licenses/by/ $4.0 /)$.

\begin{abstract}
By the end of the twentieth century, the interest in natural compounds as probable sources of drugs has declined and was replaced by other strategies such as molecular target-based drug discovery. However, in the recent times, natural compounds regained their position as extremely important source drug leads. Indole-containing compounds are under clinical use which includes vinblastine and vincristine (anticancer), atevirdine (anti-HIV), yohimbine (erectile dysfunction), reserpine (antihypertension), ajmalicine (vascular disorders), ajmaline (anti-arrhythmic), vincamine (vasodilator), etc. Monoterpene Indole Alkaloids (MIAs) deserve the curiosity and attention of researchers due to their chemical diversity and biological activities. These compounds were considered as an impending source of drug-lead. In this review 444 compounds, were identified from six genera belonging to the family Apocynaceae, will be discussed. These genera (Alstonia, Rauvolfia, Kopsia, Ervatamia, and Tabernaemontana, and Rhazya) consist of 400 members and represent $20 \%$ of Apocynaceae species. Only 30 (7.5\%) species were investigated, whereas the rest are promising to be investigated. Eleven bioactivities, including antibacterial, antifungal, anti-inflammatory and immunosuppressant activities, were reported. Whereas cytotoxic effect represents $47 \%$ of the reported activities. Convincingly, the genera selected in this review are a wealthy source for future anticancer drug lead.
\end{abstract}

Keywords: Apocynaceae; monoterpene; alkaloids; cytotoxicity; anti-inflammatory; antimicrobial

\section{Introduction}

Alkaloids are basic nitrogenous natural metabolites with structural diversity and molecular conformity. They displayed interesting bioactivities and are known to perform an important role in plant protection. The majority of them were discovered from plants and recently recorded $\mathrm{Ca} 21,000$ [1,2]. The alkaloids are generally derived from amino acids that are containing one or more nitrogen atoms. These precursors are playing a rule in their classification. Also, the biosynthetic pathway of alkaloids can be named according the amino acid source [3]. Thus, they can be categorized into several groups based on associated moieties, including piperidine, pyrrolidine, pyrrole, pyridine, quinolone, isoquinoline, indole, quinolizidine, pyrrolizidine, tropane, benzylisoquinoline, purine, $\beta$-carboline, indolinics and quinolizidine.

Terpenoids are considered to be interesting natural products that have chemical diversity and different bioactivities. Common terpenoids have been reported from marine 
sources [4]. Whereas, the plants were listed as an important source of such metabolites. Terpenoids include several subclasses according to the number of carbo-skeleton; monoterpenes $\left(C_{10}\right)$, sesquiterpenes $\left(C_{15}\right)$, diterpenes $\left(C_{20}\right)$, sesterterpenes $\left(C_{25}\right)$, triterpenes $\left(C_{30}\right)$, and tetraterpenes $\left(\mathrm{C}_{40}\right)$.

Monoterpene indole alkaloids (MIAs) are metabolites containing a bicyclic structure of a benzene ring fused to a five-membered pyrrole ring. It is a noteworthy that the occurrence of multipart alkaloids is largely restricted to limited number of plant families. (e.g., Apocynaceae, Loganiaceae, and Rubiaceae) [5-8]. These families are closely taxonomically related. Also, on the chemical aspect, they are recognized to have apparent uniformity in the building blocks of these alkaloids. MIAs have been proposed to be sourced from strictosidine, which originates from the condensation of tryptophan with secologanin ( $C_{10}$ or $C_{9}$ part), which can be divided into linear six carbon (6 C), one carbon (1 C) and three carbon (3 C) units (Figure 1). The connection between them requires proving. The nine-carbons fragment may be formed by the loss at certain stage of one of the carbons from the $3 \mathrm{C}$ unit, and there are also a few indole bases which appear to have ended up without the $3 \mathrm{C}$ or the $1 \mathrm{C}$ units. Three hypothetical building blocks, Types I, II and III. It is nevertheless a useful way of dividing indole alkaloids into groups based on their sub architecture. Since Type I alkaloids are by far the most numerous, they may be the source of Type II and III. It was suggested by LeMen and Tylor that the convention be extended to cover Type II and III alkaloids as illustrated in Figure 1. On these hypothetical bases, the MIAs categorized according to their biogenic pathway in three main groups, corynanthe, aspidosperma and iboga [9].

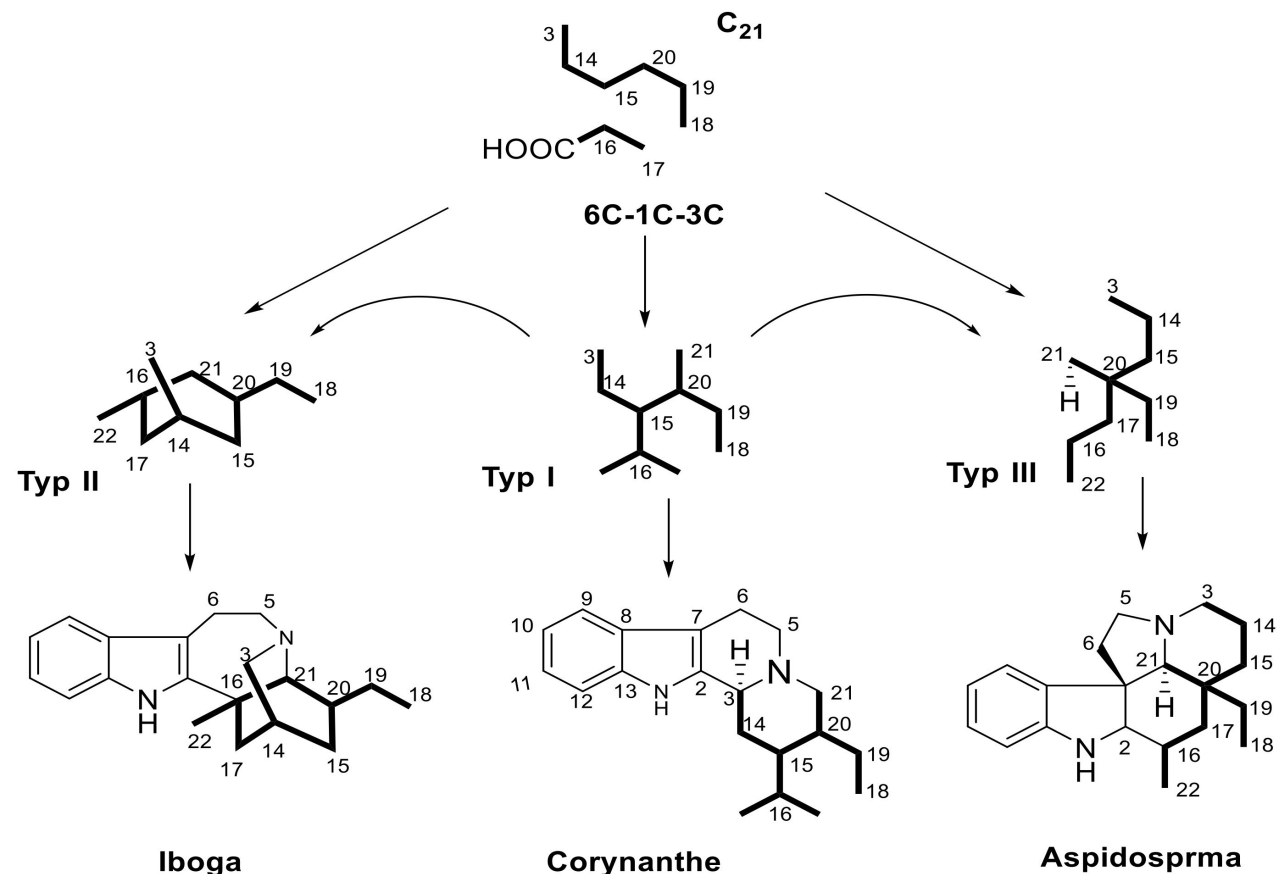

Figure 1. Biogenetic numbering rule as adopted from LeMen and Tylor.

Recently, strictosidine has been considered as the building block of MIAs biosynthesis [10]. MIAs have been proposed to arise from strictosidine, which itself originates from the condensation of tryptophan with secologanin in a 1:1 ratio. Strictosidine has been elaborated to give an impressive array of structural variants. This type of alkaloids possess 18 (or 19) carbon atoms on its skeleton. Additionally, the MIAs could be produced from tryptophan and secologanin in 1:2 or 2:1 ratio. According to this arrangement, three types (classes) of monoterpenes were constructed, including, corynanthe (e.g., ajmalicine), aspidosperma (e.g., tabersonine) and iboga (e.g., catharanthine) [11-13]. 
Apocynaceae contains about 250 genera and 2000 species [14]. Five sub-families are classified under Apocynaceae, including, Apocynoideae, Asclepiadoideae, Periplocoideae, Rauvolfioideae, and Secamonoideae. Apocynaceae species ranged from shrubs to trees. The characteristic features of these plants include colorful flowers and opposite leaves. Traditionally, species of this family have been used for the treatment of fever, malaria, gastrointestinal ailments, diabetes, and pain [15]. Additionally, some species have shown antiplasmodial and anticancer activities [14]. Several Apocynaceae MIAs have been used as anticancer, analgesic, anti-inflammatory and anti-spasmodic agents. For example, vinblastine, vinorelbine, vincristine, and vindesine were utilized as anticancer agents, whereas ajmalicine and ajmaline were used in the treatment of cardiovascular disorders (Figure 2) [2]. Catharanthus roseus and Rauvolfia serpentine are members of Apocynaceae and are known as sources of bioactive indole alkaloids [16]. Reserpine has been used as a tranquillizer, whereas vinblastine and vincristine have been used as anti-leukemic agents [17]. Vincristine and vinblastine were among the earliest anti-tumor agents, and since 1965 have been used as tubulin polymerization inhibitors. They have been used in combination for the treatment of acute lymphoblastic leukemia and also against both Hodgkin's and non- Hodgkin lymphoma. Additionally, strychnine is potent muscle contracting agent whereas, yohimbine has been used for the treatment of sexual dysfunction and investigated as a remedy for type-2 diabetes in animal and human models.

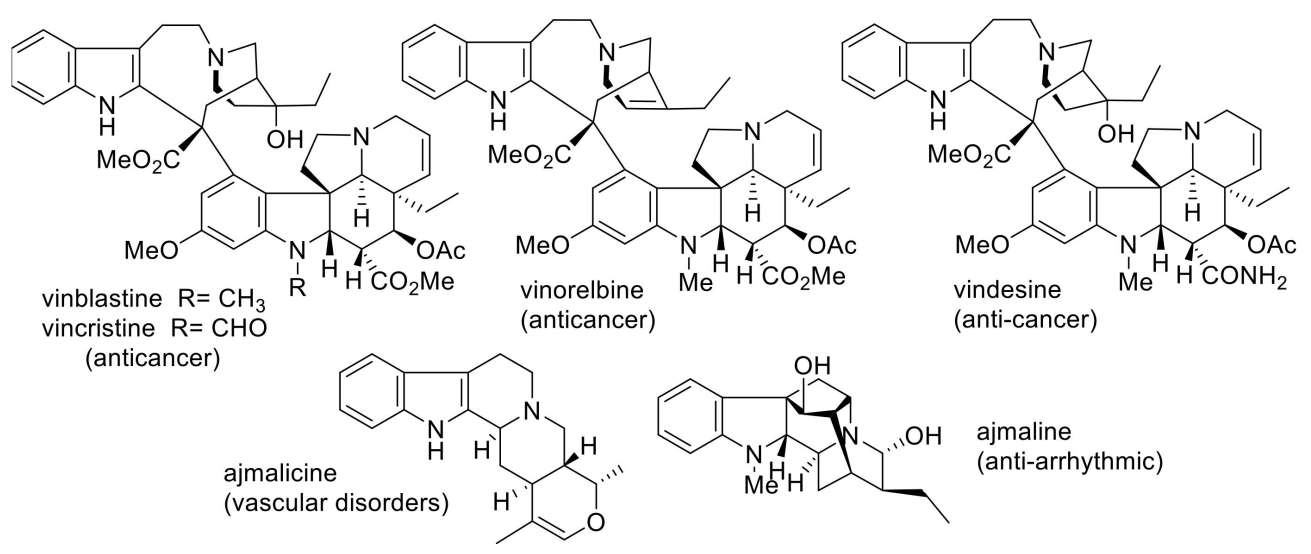

Figure 2. Examples of well-known biologically active terpene indole alkaloids.

There are several publications interested in the terpene indole alkaloids of individual species of the family Apocynaceae. The current review organizes the reported MIAs considering the historical aspect in each selected genus. Moreover, these MIAs were biosynthetically classified according to the tepenoidal fragment, i.e., corynanthe, aspidosperma, or iboga. Also, it focuses on the origin, structural diversity and biological activities exerted by 444 (Table 1) monoterpene indole alkaloids which have been reported from selected six genera of the family Apocynaceae (Alstonia, Kopsia, Ervatamia, Rauvolfia, Tabernaemontana and Rhazya), in the period between 2010 and December 2020. The listed metabolites are categorized under 26 subclasses, ajmaline, akuamiline, akuammidine, akuammicine, apparicine, aspidofractinine, aspidospermatan, eburnane, flabelliformide, kopsine, macroline, macroline oxindole, macroline-akuammiline, methyl chanofruticosinate, nareline, paucidactine, picrinine, pleiocarpamine, sarpagine, scholaricine, secodine, strictosidine, strychnos, vincamine, vincorine and vobasine (Figures 3 and 4). 
Table 1. Monoterpenoid indole alkaloids from the six species of Apocynaceae.

\begin{tabular}{|c|c|c|c|c|c|c|}
\hline Comp No & Compound Name & Class Type & Source & Part & Country & Activities \\
\hline 1 & (14a,15a)-14,15-Epoxy Aspidofractinine & Aspidofractinine & Alstonia mairei & Leaves and twigs & China & Cytotoxicity \\
\hline 2 & Maireine A & Aspidosperma & Alstonia mairei & Leaves and twigs & China & Cytotoxicity \\
\hline 3 & Maireine B & Aspidosperma & Alstonia mairei & Leaves and twigs & China & Cytotoxicity \\
\hline 4 & Venalstonine & Aspidofractinine & Alstonia mairei & Leaves and twigs & China & Cytotoxicity \\
\hline 5 & (-)-Minovincinine & Aspidosperma & Alstonia mairei & Leaves and twigs & China & Cytotoxicity \\
\hline 6 & (-)-11-Methoxymino Vincinine & Aspidosperma & Alstonia mairei & Leaves and twigs & China & Cytotoxicity \\
\hline 7 & (-)-Echitovenine & Aspidosperma & Alstonia mairei & Leaves and twigs & China & Cytotoxicity \\
\hline 8 & Echitovenaldine & Aspidosperma & Alstonia mairei & Leaves and twigs & China & Cytotoxicity \\
\hline 9 & Echitovenidine & Aspidosperma & Alstonia mairei & Leaves and twigs & China & Cytotoxicity \\
\hline 10 & 11-Methoxyechitovenidine & Aspidosperma & Alstonia mairei & Leaves and twigs & China & Cytotoxicity \\
\hline 11 & Echitoveniline & Aspidosperma & Alstonia mairei & Leaves and twigs & China & Cytotoxicity \\
\hline 12 & 11-Methoxyechitoveniline & Aspidosperma & Alstonia mairei & Leaves and twigs & China & Cytotoxicity \\
\hline 13 & Echitoserpidine & Aspidosperma & Alstonia mairei & Leaves and twigs & China & Cytotoxicity \\
\hline 14 & 11-Methoxyechitoserpidine & Aspidosperma & Alstonia mairei & Leaves and twigs & China & Cytotoxicity \\
\hline 16 & Lochnericine & Aspidosperma & Alstonia mairei & Leaves and twigs & China & Cytotoxicity \\
\hline 17 & Tabersonine & Aspidosperma & Alstonia mairei & Leaves and twigs & China & Cytotoxicity \\
\hline 18 & Perakine & Ajmaline & Alstonia mairei & Leaves and twigs & China & Cytotoxicity \\
\hline 19 & Picrinine & Picrinine & Alstonia mairei & Leaves and twigs & China & Cytotoxicity \\
\hline 20 & Deacetylpicraline 3,4,5-Trimethoxybenzoate & picraline & Alstonia mairei & Leaves and twigs & China & Cytotoxicity \\
\hline 21 & Picralinal & picraline & Alstonia mairei & Leaves and twigs & China & Cytotoxicity \\
\hline 22 & Rhazimol & Akummidine & Alstonia mairei & Leaves and twigs & China & Cytotoxicity \\
\hline 23 & Alsmaphorazines A & Scholaricine & Alstonia pneumatophore & Leaves & Malysia & Anti-inflammatory \\
\hline 24 & Alsmaphorazine B & Scholaricine & Alstonia pneumatophore & Leaves & Malysia & Anti-inflammatory \\
\hline 25 & Alstrostine A & Strictosidine & Alstonia rostrata & Leaves and twigs & China & Cytotoxicity \\
\hline 26 & Alstrostine B & Strictosidine & Alstonia rostrata & Leaves and twigs & China & Cytotoxicity \\
\hline
\end{tabular}


Table 1. Cont

\begin{tabular}{|c|c|c|c|c|c|c|}
\hline Comp No & Compound Name & Class Type & Source & Part & Country & Activities \\
\hline 27 & Alstrostine C & Akummicine & Alstonia rostrata & Leaves and twigs & China & Cytotoxicity \\
\hline 28 & Alstrostine D & Akummicine & Alstonia rostrata & Leaves and twigs & China & Cytotoxicity \\
\hline 29 & Alstrostine E & Akummicine & Alstonia rostrata & Leaves and twigs & China & Cytotoxicity \\
\hline 30 & Alstrostine F & Corynanthe & Alstonia rostrata & Leaves and twigs & China & Cytotoxicity \\
\hline 31 & $\begin{array}{l}\text { 11-Hydroxy-6,7-Epoxy-8-Oxo- } \\
\text { Vincadifformine }\end{array}$ & Aspidosperma & Alstonia yunnanensis & Whole plant & China & Cytotoxicity \\
\hline 32 & 14-Chloro-15-Hydroxyvinca Difformine & Aspidosperma & Alstonia yunnanensis & Whole plant & China & Cytotoxicity \\
\hline 33 & Perakine $\mathrm{N}_{4}$-Oxide & Ajmaline & Alstonia yunnanensis & Whole plant & China & Cytotoxicity \\
\hline 34 & Raucaffrinoline $\mathrm{N}_{4}$-Oxide & Ajmaline & Alstonia yunnanensis & Whole plant & China & Cytotoxicity \\
\hline 35 & Vinorine $N_{1}, N_{4}$-Dioxide & Ajmaline & Alstonia yunnanensis & Whole plant & China & Cytotoxicity \\
\hline 36 & Oxovincadifformine & Aspidosperma & Alstonia yunnanensis & Whole plant & China & Cytotoxicity \\
\hline 37 & Vinorine $N_{4}$-Oxide & Ajmaline & Alstonia yunnanensis & Whole plant & China & Cytotoxicity \\
\hline 38 & Vinorine & Ajmaline & Alstonia yunnanensis & Whole plant & China & Cytotoxicity \\
\hline 39 & Alsmaphorazine C & $\begin{array}{l}\text { Octahydropyrrolo[2,3-b]pyrrole } \\
\text { and 2-azabicyclo[3.3.1]nonane } \\
\text { units }\end{array}$ & Alstonia pneumatophore & Leaves & Malaysia & Cytotoxicity \\
\hline 40 & Alsmaphorazine D & $\begin{array}{l}\text { Octahydropyrrolo[2,3-b]pyrrole } \\
\text { and } \\
\text { 2,8-diazabicyclo[3.3.1]nonane } \\
\text { units }\end{array}$ & Alstonia pneumatophore & Leaves & Malaysia & Cytotoxicity \\
\hline 41 & Alsmaphorazine E & $\begin{array}{c}\text { Octahydropyrrolo[2,3-b]pyrrole } \\
\text { and } \\
\text { 2,8-diazabicyclo[3.3.1]nonane } \\
\text { units }\end{array}$ & Alstonia pneumatophore & Leaves & Malaysia & Cytotoxicity \\
\hline 42 & Scholarisin I & picraline & Alstonia scholaris & Leaves & China & $\begin{array}{c}\text { Cytotoxicity, } \\
\text { Anti-inflammatory } \\
\text { Antifungal }\end{array}$ \\
\hline
\end{tabular}


Table 1. Cont.

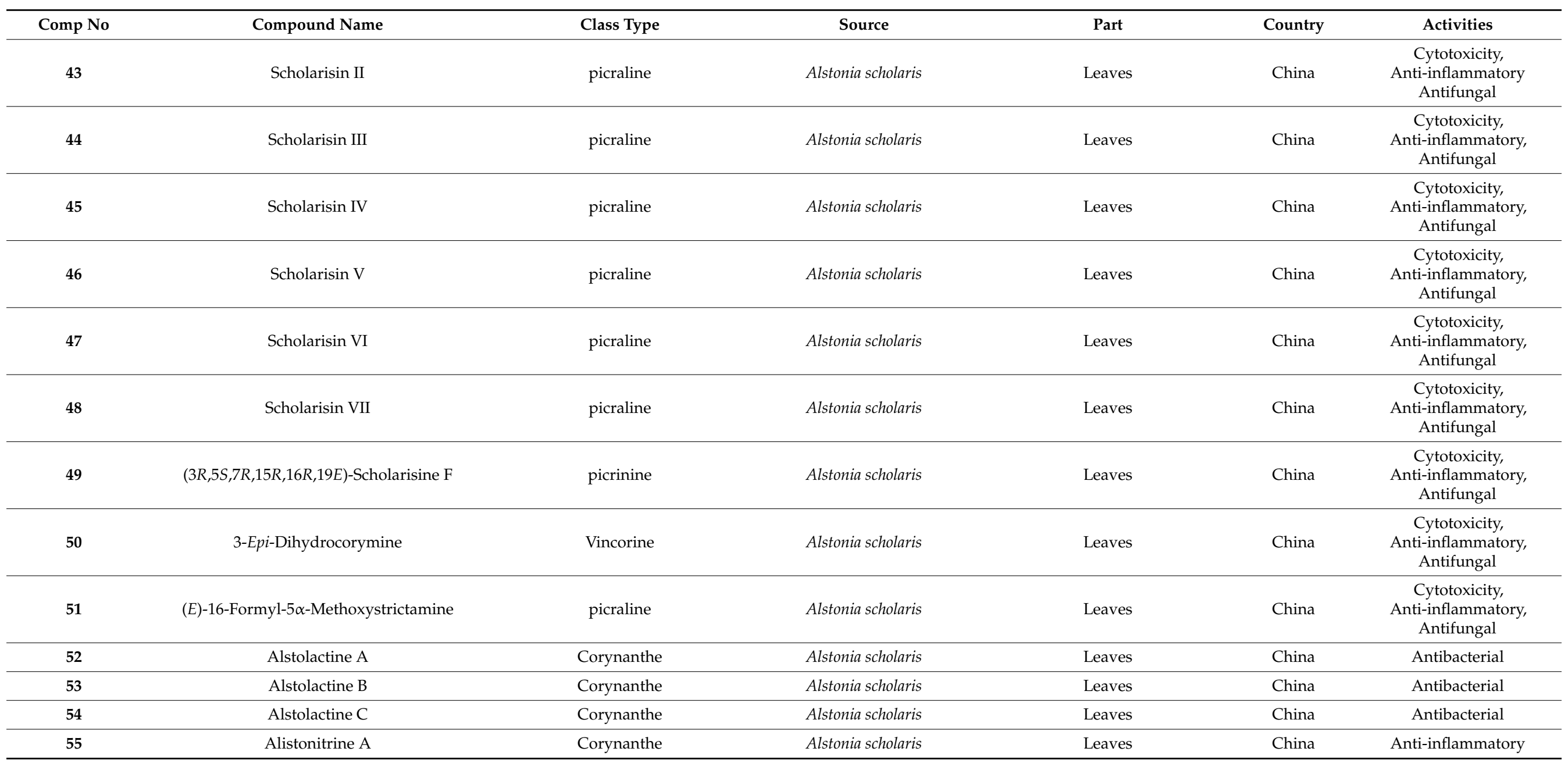


Table 1. Cont.

\begin{tabular}{|c|c|c|c|c|c|c|}
\hline Comp No & Compound Name & Class Type & Source & Part & Country & Activities \\
\hline 56 & 6,7-Epoxy-8-Oxo-Vincadifformine & Aspidosperma & Alstonia rupestris & $\begin{array}{c}\text { Aerial } \\
\text { parts }\end{array}$ & China & $\begin{array}{c}\text { Cytotoxicity, Antifungal, } \\
\text { Antibacterial }\end{array}$ \\
\hline 57 & 11-Acetyl-6,7-Epoxy-8-Oxo-Vincadifformine & Aspidosperma & Alstonia rupestris & $\begin{array}{c}\text { Aerial } \\
\text { parts }\end{array}$ & China & $\begin{array}{c}\text { Cytotoxicity, Antifungal, } \\
\text { Antibacterial }\end{array}$ \\
\hline 58 & $\begin{array}{l}\text { 11-Hydroxy-14-Chloro-15- } \\
\text { Hydroxyvincadifformine }\end{array}$ & Aspidosperma & Alstonia rupestris & $\begin{array}{c}\text { Aerial } \\
\text { parts }\end{array}$ & China & $\begin{array}{c}\text { Cytotoxicity, Antifungal, } \\
\text { Antibacterial }\end{array}$ \\
\hline 59 & Perakine $N_{1}, N_{4}$-Dioxide & Ajmaline & Alstonia rupestris & $\begin{array}{c}\text { Aerial } \\
\text { parts }\end{array}$ & China & $\begin{array}{c}\text { Cytotoxicity, Antifungal, } \\
\text { Antibacterial }\end{array}$ \\
\hline 60 & 11-Hydroxy-6,7-Epoxy-8-Oxovincadifformine & Aspidosperma & Alstonia rupestris & $\begin{array}{l}\text { Aerial } \\
\text { parts }\end{array}$ & China & $\begin{array}{c}\text { Cytotoxicity, Antifungal, } \\
\text { Antibacterial }\end{array}$ \\
\hline 61 & $N_{(4)}$-Methyl-Talpinine & Sarpagine & Alstonia angustifolia & $\begin{array}{l}\text { Stem } \\
\text { bark }\end{array}$ & Vietnam & $\begin{array}{l}\text { Anti-inflammatory, } \\
\text { Anti-Leishmanial }\end{array}$ \\
\hline 62 & $N_{(4)}$-Meth-Yl- $N_{(4)}, 21-S e c o t a l p i n i n e$ & Macroline & Alstonia angustifolia & $\begin{array}{l}\text { Stem } \\
\text { bark }\end{array}$ & Vietnam & $\begin{array}{l}\text { Anti-inflammatory, } \\
\text { Anti-Leishmanial }\end{array}$ \\
\hline 63 & Alstonerinal & Macroline & Alstonia angustifolia & $\begin{array}{l}\text { Stem } \\
\text { bark }\end{array}$ & Vietnam & $\begin{array}{l}\text { Anti-inflammatory, } \\
\text { Anti-Leishmanial }\end{array}$ \\
\hline 64 & Alstonerine & Macroline & Alstonia angustifolia & $\begin{array}{l}\text { Stem } \\
\text { bark }\end{array}$ & Vietnam & $\begin{array}{l}\text { Anti-inflammatory, } \\
\text { Anti-Leishmanial }\end{array}$ \\
\hline 65 & Macrocarpine B & Macroline & Alstonia angustifolia & $\begin{array}{l}\text { Stem } \\
\text { bark }\end{array}$ & Vietnam & $\begin{array}{l}\text { Anti-inflammatory, } \\
\text { Anti-Leishmanial }\end{array}$ \\
\hline 66 & Affinisine & Sarpagine & Alstonia angustifolia & $\begin{array}{l}\text { Stem } \\
\text { bark }\end{array}$ & Vietnam & $\begin{array}{l}\text { Anti-inflammatory, } \\
\text { Anti-Leishmanial }\end{array}$ \\
\hline 67 & Villalstonine & Macroline-Pleiocarpamine & Alstonia angustifolia & $\begin{array}{l}\text { Stem } \\
\text { bark }\end{array}$ & Vietnam & $\begin{array}{l}\text { Anti-inflammatory, } \\
\text { Anti-Leishmanial }\end{array}$ \\
\hline 68 & Villalstonine $N_{(4)}$-Oxide & Macroline-Pleiocarpamine & Alstonia angustifolia & $\begin{array}{l}\text { Stem } \\
\text { bark }\end{array}$ & Vietnam & $\begin{array}{l}\text { Anti-inflammatory, } \\
\text { Anti-Leishmanial }\end{array}$ \\
\hline 69 & Villalstonidine D & Macroline-Pleiocarpamine & Alstonia angustifolia & $\begin{array}{l}\text { Stem } \\
\text { bark }\end{array}$ & Vietnam & $\begin{array}{l}\text { Anti-inflammatory, } \\
\text { Anti-Leishmanial }\end{array}$ \\
\hline 70 & Villalstonidine E & Macroline-Pleiocarpamine & Alstonia angustifolia & $\begin{array}{l}\text { Stem } \\
\text { bark }\end{array}$ & Vietnam & $\begin{array}{l}\text { Anti-inflammatory, } \\
\text { Anti-Leishmanial }\end{array}$ \\
\hline
\end{tabular}


Table 1. Cont.

\begin{tabular}{|c|c|c|c|c|c|c|}
\hline Comp No & Compound Name & Class Type & Source & Part & Country & Activities \\
\hline 71 & Normavacurine-21-One & Pleiocarpaman & Alstonia scholaris & Leaves & China & Antibacterial \\
\hline 72 & 5-Hydroxy-19,20-E-Alschomine & Corynanthe & Alstonia scholaris & Leaves & China & Antibacterial \\
\hline 73 & 5-Hydroxy-19,20-Z-Alschomine & Corynanthe & Alstonia scholaris & Leaves & China & Antibacterial \\
\hline 74 & Alstoniascholarine A & Corynanthe & Alstonia scholaris & Leaves & China & $\begin{array}{l}\text { Antibacterial, } \\
\text { Anti- Fungal }\end{array}$ \\
\hline 75 & Alstoniascholarine B & Corynanthe & Alstonia scholaris & Leaves & China & $\begin{array}{l}\text { Antibacterial, Anti- } \\
\text { Fungal }\end{array}$ \\
\hline 77 & Alstoniascholarine D & Corynanthe & Alstonia scholaris & Leaves & China & $\begin{array}{l}\text { Antibacterial, } \\
\text { Anti- Fungal }\end{array}$ \\
\hline 78 & Alstoniascholarine E & Corynanthe & Alstonia scholaris & Leaves & China & $\begin{array}{l}\text { Antibacterial, } \\
\text { Anti- Fungal }\end{array}$ \\
\hline 79 & Alstoniascholarine $\mathrm{F}$ & Scholarisine & Alstonia scholaris & Leaves & China & $\begin{array}{l}\text { Antibacterial, } \\
\text { Anti- Fungal }\end{array}$ \\
\hline 80 & Alstoniascholarine $\mathrm{G}$ & Scholarisine & Alstonia scholaris & Leaves & China & $\begin{array}{l}\text { Antibacterial, } \\
\text { Anti- Fungal }\end{array}$ \\
\hline 81 & Alstoniascholarine $\mathrm{H}$ & Scholarisine & Alstonia scholaris & Leaves & China & $\begin{array}{l}\text { Antibacterial, } \\
\text { Anti- Fungal }\end{array}$ \\
\hline 82 & Alstoniascholarine I & Scholarisine & Alstonia scholaris & Leaves & China & $\begin{array}{l}\text { Antibacterial, } \\
\text { Anti- Fungal }\end{array}$ \\
\hline 83 & Alstoniascholarine J & Scholarisine & Alstonia scholaris & Leaves & China & $\begin{array}{l}\text { Antibacterial, } \\
\text { Anti- Fungal }\end{array}$ \\
\hline 84 & Alstoniascholarine $\mathrm{K}$ & Scholarisine & Alstonia scholaris & Leaves & China & $\begin{array}{l}\text { Antibacterial, } \\
\text { Anti- Fungal }\end{array}$ \\
\hline 85 & Alstoniascholarine L & Corynanthe & Alstonia scholaris & Leaves & China & Cytotoxicity \\
\hline 86 & Alstoniascholarine M & Corynanthe & Alstonia scholaris & Leaves & China & Cytotoxicity \\
\hline 87 & Alstoniascholarine $\mathrm{N}$ & Corynanthe & Alstonia scholaris & Leaves & China & Cytotoxicity \\
\hline 88 & Alstoniascholarine $\mathrm{O}$ & Scholarisine & Alstonia scholaris & Leaves & China & Cytotoxicity \\
\hline 89 & Alstoniascholarine $\mathrm{P}$ & Scholarisine & Alstonia scholaris & Leaves & China & Cytotoxicity \\
\hline
\end{tabular}


Table 1. Cont.

\begin{tabular}{|c|c|c|c|c|c|c|}
\hline Comp No & Compound Name & Class Type & Source & Part & Country & Activities \\
\hline 90 & Alstoniascholarine Q & Scholarisine & Alstonia scholaris & Leaves & China & Cytotoxicity \\
\hline 91 & Scholarisine $\mathrm{H}$ & Corynanthe & Alstonia scholaris & Leaves & China & Antibacterial \\
\hline 92 & Scholarisine I & Nareline & Alstonia scholaris & Leaves & China & Antibacterial \\
\hline 93 & Scholarisine J & Nareline & Alstonia scholaris & Leaves & China & Antibacterial \\
\hline 94 & Scholarisine K & Corynanthe & Alstonia scholaris & Leaves & China & Antibacterial \\
\hline 95 & Scholarisine L & Corynanthe & Alstonia scholaris & Leaves & China & Antibacterial \\
\hline 97 & Scholarisine N & Corynanthe & Alstonia scholaris & Leaves & China & Antibacterial \\
\hline 98 & Melosline A & Corynanthe & Alstonia scholaris & Leaves and twigs & China & Cytotoxicity \\
\hline 99 & Melosline B & Secodine & Alstonia scholaris & Leaves and twigs & China & Cytotoxicity \\
\hline 100 & $\begin{array}{l}\text { 1-[2-[2-(Carboxymethyl) Indole-3-Yl] } \\
\text { Ethyl]-3-Ethylpyridinium Hydroxide } \\
\text { Inner Salt }\end{array}$ & Secodine & Alstonia scholaris & Leaves and twigs & China & Cytotoxicity \\
\hline 101 & Alstiyunnanenine A & Sarpagine & Alstonia Yunnanensis & $\begin{array}{c}\text { Aerial } \\
\text { parts }\end{array}$ & China & Cytotoxicity \\
\hline 103 & Alstiyunnanenine $\mathrm{C}$ & Akummiline & Alstonia Yunnanensis & $\begin{array}{c}\text { Aerial } \\
\text { parts }\end{array}$ & China & Cytotoxicity \\
\hline 104 & Alstiyunnanenine D & Scholaricine & Alstonia Yunnanensis & $\begin{array}{l}\text { Aerial } \\
\text { parts }\end{array}$ & China & Cytotoxicity \\
\hline 105 & Alstiyunnanenine E & Scholaricine & Alstonia Yunnanensis & $\begin{array}{c}\text { Aerial } \\
\text { parts }\end{array}$ & China & Cytotoxicity \\
\hline 106 & Alstomairine A & Scholaricine & Alstonia Mairei & Leaves & China & Cytotoxicity \\
\hline 107 & Alstomairine B & Scholaricine & Alstonia Mairei & Leaves & China & Cytotoxicity \\
\hline 108 & Alstomairine $\mathrm{C}$ & Scholaricine & Alstonia Mairei & Leaves & China & Cytotoxicity \\
\hline 109 & Alpneumine A & Scholaricine & Alstonia Mairei & Leaves & China & Cytotoxicity \\
\hline
\end{tabular}


Table 1. Cont.

\begin{tabular}{|c|c|c|c|c|c|c|}
\hline Comp No & Compound Name & Class Type & Source & Part & Country & Activities \\
\hline 110 & Alstrostine $\mathrm{G}$ & Corynanthe & Alstonia rostrata & Bark and trunks & China & Cytotoxicity \\
\hline 111 & Alstrostine $\mathrm{H}$ & Corynanthe & Alstonia rostrata & Bark and trunks & China & Cytotoxicity \\
\hline 112 & Alstrostine I & Scholarisine & Alstonia rostrata & Bark and trunks & China & Cytotoxicity \\
\hline 113 & Alstrostine J & Secodine & Alstonia rostrata & Bark and trunks & China & Cytotoxicity \\
\hline 114 & Alstrostine K & Corynanthe & Alstonia rostrata & Bark and trunks & China & Cytotoxicity \\
\hline 115 & Scholarisine $\mathrm{T}$ & Nareline & Alstonia scholaris & Leaves & China & Antibacterial \\
\hline 116 & Scholarisine U & Nareline & Alstonia scholaris & Leaves & China & Antibacterial \\
\hline 117 & Scholarisine V & Nareline & Alstonia scholaris & Leaves & China & Antibacterial \\
\hline 118 & Scholarisine W & Nareline & Alstonia scholaris & Leaves & China & Antibacterial \\
\hline 119 & Scholarisine A & Nareline & Alstonia scholaris & Leaves & China & Antibacterial \\
\hline 120 & Scholarisine P & Nareline & Alstonia scholaris & Leaves & China & Anti-inflammatory \\
\hline 121 & Scholarisine Q & Akuammiline & Alstonia scholaris & Leaves & China & Anti-inflammatory \\
\hline 122 & Scholarisine R & Corynanthe & Alstonia scholaris & Leaves & China & Anti-inflammatory \\
\hline 123 & Scholarisine S & Nareline & Alstonia scholaris & Leaves & China & Anti-inflammatory \\
\hline 125 & Nareline & Nareline & Alstonia scholaris & Leaves & China & Anti-inflammatory \\
\hline 126 & 5-Methoxystrictamine & Akuammiline & Alstonia scholaris & Leaves & China & Anti-inflammatory \\
\hline 127 & Leuconolam & Aspidosperma & Alstonia scholaris & Leaves & China & Anti-inflammatory \\
\hline 128 & Epileuconolam & Aspidosperma & Alstonia scholaris & Leaves & China & Anti-inflammatory \\
\hline 129 & $N_{\mathrm{b}}$-Demethylalstogustine & Scholarisine & Alstonia scholaris & Leaves & China & Anti-inflammatory \\
\hline 130 & 19-Epischolaricine & Scholarisine & Alstonia scholaris & Leaves & China & Anti-inflammatory \\
\hline 131 & Scholaricine & Scholarisine & Alstonia scholaris & Leaves & China & Anti-inflammatory \\
\hline 132 & Vallesamine & Corynanthe & Alstonia scholaris & Leaves & China & Anti-inflammatory \\
\hline 133 & Akuammidine & Akuammidine & Alstonia scholaris & Leaves & China & Anti-inflammatory \\
\hline 134 & 17-Nor-Excelsinidine & Corynanthe & Alstonia scholaris & Leaves & China & Anti-inflammatory \\
\hline 135 & Strictosamide & Corynanthe & Alstonia scholaris & Leaves & China & Anti-inflammatory \\
\hline
\end{tabular}


Table 1. Cont.

\begin{tabular}{|c|c|c|c|c|c|c|}
\hline Comp No & Compound Name & Class Type & Source & Part & Country & Activities \\
\hline 136 & Vincamaginine A & Ajmaline & Alstonia penangiana & Leaves & Malaysia & Cytotoxicity \\
\hline 137 & Vincamaginine B & Ajmaline & Alstonia penangiana & Leaves & Malaysia & Cytotoxicity \\
\hline 138 & Alstonisinine A & Macroline Oxindole & Alstonia penangiana & Leaves & Malaysia & Cytotoxicity \\
\hline 139 & Alstonisinine B & Macroline Oxindole & Alstonia penangiana & Leaves & Malaysia & Cytotoxicity \\
\hline 140 & Alstonisinine $\mathrm{C}$ & Macroline Oxindole & Alstonia penangiana & Leaves & Malaysia & Cytotoxicity \\
\hline 141 & Alstonoxine F & Macroline Oxindole & Alstonia penangiana & Leaves & Malaysia & Cytotoxicity \\
\hline 143 & Angustilongine B & Macroline-Akuammiline & Alstonia penangiana & Leaves & Malaysia & Cytotoxicity \\
\hline 144 & Angustilongine $\mathrm{C}$ & Macroline-Akuammiline & Alstonia penangiana & Leaves & Malaysia & Cytotoxicity \\
\hline 145 & Angustilongine D & Macroline-Akuammiline & Alstonia penangiana & Leaves & Malaysia & Cytotoxicity \\
\hline 146 & Winphyllines A & Vincorine & Alstonia rostrata & Twigs & China & Cytotoxicity \\
\hline 147 & Winphyllines B & Scholarisine & Alstonia rostrata & Twigs & China & Cytotoxicity \\
\hline 148 & $N_{\mathrm{b}}$-Demethylechitamine & Vincorine & Alstonia rostrata & Twigs & China & Cytotoxicity \\
\hline 149 & 17-O-Acetylnorechitamine & Vincorine & Alstonia rostrata & Twigs & China & Cytotoxicity \\
\hline 151 & $N_{(4)}$-Demethylastogustine & Scholarisine & Alstonia rostrata & Twigs & China & Cytotoxicity \\
\hline 152 & 17-Formyl-10-Demethoxyvincorine $N_{(4)}$-Oxide & Vincorine & Alstonia scholaris & Leaves & China & - \\
\hline 153 & 10-Methoxyalstiphyllanine $\mathrm{H}$ & Ajmaline & Alstonia scholaris & Leaves & China & - \\
\hline 154 & 10-Demethoxyvincorine $N_{(4)}$-Oxide & Vincorine & Alstonia scholaris & Leaves & China & - \\
\hline 155 & Alstoscholactine & Corynanthe & Alstonia scholaris & Leaves & Malaysia & $\begin{array}{c}\text { Vasorelaxation } \\
\text { Cytotoxicity }\end{array}$ \\
\hline 156 & Alstolaxepine & Corynanthe & Alstonia scholaris & Leaves & Malaysia & $\begin{array}{c}\text { Vasorelaxation } \\
\text { Cytotoxicity }\end{array}$ \\
\hline 157 & Alstobrogaline & Corynanthe & Alstonia scholaris & Leaves & Malaysia & Cytotoxicity \\
\hline 158 & Kopsiyunnanines G & Aspidosperma & Kopsia arbora & $\begin{array}{l}\text { Aerial } \\
\text { parts }\end{array}$ & China & - \\
\hline
\end{tabular}


Table 1. Cont.

\begin{tabular}{|c|c|c|c|c|c|c|}
\hline Comp No & Compound Name & Class Type & Source & Part & Country & Activities \\
\hline 159 & Kopsiyunnanines $\mathrm{H}$ & Aspidosperma & Kopsia arbora & $\begin{array}{c}\text { Aerial } \\
\text { parts }\end{array}$ & China & - \\
\hline 160 & Kopsihainin A & Aspidosperma & Kopsia hainanensis & Stems & China & Antitussive \\
\hline 161 & Kopsihainin B & Aspidofractinine & Kopsia hainanensis & Stems & China & Antitussive \\
\hline 162 & Kopsihainin C & Aspidofractinine & Kopsia hainanensis & Stems & China & Antitussive \\
\hline 163 & Kopsinine & Aspidofractinine & Kopsia hainanensis & Stems & China & Antitussive \\
\hline 164 & Methyl Demethoxycarbonylchanofruticosinate & Methyl Chanofruticosinate & Kopsia hainanensis & Stems & China & Antitussive \\
\hline 165 & Singaporentine A & Aspidofractinine & Kopsia singapurensis & Barks and leaves & Malaysia & - \\
\hline 166 & $N_{(1)}$-Formylkopsininic Acid & Aspidofractinine & Kopsia singapurensis & Barks and leaves & Malaysia & - \\
\hline 167 & $N_{(1)}$-Formylkopsininic Acid- $N_{(4)}$-Oxide & Aspidofractinine & Kopsia singapurensis & Barks and leaves & Malaysia & - \\
\hline 168 & 15-Hydroxykopsamine & Aspidofractinine & Kopsia singapurensis & Barks and leaves & Malaysia & - \\
\hline 169 & $14 \alpha$-Hydroxy- $N_{(4)}$-Methylcondylocarpine & Aspidosoermata & Kopsia singapurensis & Barks and leaves & Malaysia & - \\
\hline 170 & Singaporentinidine & Corynanthe & Kopsia singapurensis & Barks and leaves & Malaysia & - \\
\hline 171 & Kopsininate & Aspidofractinie & Kopsia hainanensis & Leaves and stems & China & Antifungal, Antibacterial \\
\hline 173 & $\begin{array}{l}\text { Methyl } N_{1} \text { - Decarbomethoxy } \\
\text { Chanofruticosinate } N_{(4)} \text {-Oxide }\end{array}$ & Methyl Chanofruticosinate & Kopsia hainanensis & Leaves and stems & China & Antifungal, Antibacterial \\
\hline 174 & Methyl Chanofruticosinate $N_{(4)}$-Oxide & Methyl Chanofruticosinate & Kopsia hainanensis & Leaves and stems & China & Antifungal, Antibacterial \\
\hline 175 & 5,6-Secokopsinine & Aspidofractinine & Kopsia jasminiflora & Stem barks & Thailand & Cytotoxicity \\
\hline 176 & $5 \beta$-Hydroxykopsinine & Aspidofractinine & Kopsia jasminiflora & Stem barks & Thailand & Cytotoxicity \\
\hline 177 & 16-Epi-Kopsinilam & Aspidofractinine & Kopsia jasminiflora & Stem barks & Thailand & Cytotoxicity \\
\hline 178 & 5-Oxokopsinic Acid & Aspidofractinine & Kopsia jasminiflora & Stem barks & Thailand & Cytotoxicity \\
\hline 179 & $N_{\mathrm{a}}$-Demethoxycarbonyl-12-Methoxykopsine & Kopsine & Kopsia jasminiflora & Stem barks & Thailand & Cytotoxicity \\
\hline 180 & 14(S)-Hydroxy-19(R)- Methoxytubotaiwine & Strychnos & Kopsia jasminiflora & Stem barks & Thailand & Cytotoxicity \\
\hline 181 & 19-Oxo-(-)-Eburnamonine & Vincamine & Kopsia jasminiflora & Stem barks & Thailand & Cytotoxicity \\
\hline 182 & $19(S)$-Hydroxy- $\Delta^{14}$-Vincamone & Vincamine & Kopsia jasminiflora & Stem barks & Thailand & Cytotoxicity \\
\hline
\end{tabular}


Table 1. Cont.

\begin{tabular}{|c|c|c|c|c|c|c|}
\hline Comp No & Compound Name & Class Type & Source & Part & Country & Activities \\
\hline 183 & Kopsinilam & Aspidofractinine & Kopsia jasminiflora & Stem barks & Thailand & Cytotoxicity \\
\hline 184 & Kopsinic Acid & Aspidofractinine & Kopsia jasminiflora & Stem barks & Thailand & Cytotoxicity \\
\hline 185 & 12-Methoxykopsine & Kopsine & Kopsia jasminiflora & Stem barks & Thailand & Cytotoxicity \\
\hline 186 & Kopsanone & Kopsine & Kopsia jasminiflora & Stem barks & Thailand & Cytotoxicity \\
\hline 187 & 19(R)- Methoxytubotaiwine & Strychnos & Kopsia jasminiflora & Stem barks & Thailand & Cytotoxicity \\
\hline 188 & (-)-Eburnamonine & Vincamine & Kopsia jasminiflora & Stem barks & Thailand & Cytotoxicity \\
\hline 189 & 19-OH-(-)-Eburnamonine & Vincamine & Kopsia jasminiflora & Stem barks & Thailand & $\begin{array}{c}\text { Cytotoxicity, } \\
\text { Acetylcholinesterase } \\
\text { inhibitor }\end{array}$ \\
\hline 190 & $\Delta^{14}$-Vincamone & Vincamine & Kopsia jasminiflora & Stem barks & Thailand & Cytotoxicity \\
\hline 191 & Phutdonginin & Eburnane & Kopsia arborea & Twigs & Thailand & $\begin{array}{c}\text { Antibacterial, } \\
\text { Acetylcholinesterase } \\
\text { inhibition }\end{array}$ \\
\hline 192 & Melodinine E & Aspidosperma & Kopsia arborea & Twigs & Thailand & $\begin{array}{c}\text { Antibacterial, } \\
\text { Acetylcholinesterase } \\
\text { inhibition }\end{array}$ \\
\hline 194 & Kopsamine & Aspidofractinine & Kopsia arborea & Twigs & Thailand & $\begin{array}{c}\text { Antibacterial, } \\
\text { Acetylcholinesterase } \\
\text { inhibition }\end{array}$ \\
\hline 195 & (-)-Methylenedioxy-11,12-Kopsinaline & Aspidofractinine & Kopsia arborea & Twigs & Thailand & $\begin{array}{c}\text { Antibacterial, } \\
\text { Acetylcholinesterase } \\
\text { inhibition }\end{array}$ \\
\hline 196 & Decarbomethoxykopsiline & Kopsine & Kopsia arborea & Twigs & Thailand & $\begin{array}{c}\text { Antibacterial, } \\
\text { Acetylcholinesterase } \\
\text { inhibition }\end{array}$ \\
\hline
\end{tabular}


Table 1. Cont.

\begin{tabular}{|c|c|c|c|c|c|c|}
\hline Comp No & Compound Name & Class Type & Source & Part & Country & Activities \\
\hline 197 & Vincadifformine & Aspidosperma & Kopsia arborea & Twigs & Thailand & $\begin{array}{c}\text { Antibacterial, } \\
\text { Acetylcholinesterase } \\
\text { inhibition }\end{array}$ \\
\hline 199 & Kopsiyunnanines J1 and J2 & Aspidosoermata & Kopsia arborea & $\begin{array}{l}\text { Aerial } \\
\text { parts }\end{array}$ & China & - \\
\hline 200 & Paucidirinine & Aspidofractinine & Kopsia pauciflora & Stem bark & Malaysia & Cytotoxicity \\
\hline 202 & Paucidactinine & Aspidosperma & Kopsia pauciflora & Stem bark & Malaysia & Cytotoxicity \\
\hline 203 & Pauciduridine & Aspidofractinine & Kopsia pauciflora & Stem bark & Malaysia & Cytotoxicity \\
\hline 204 & Paucidactine D & Paucidactine & Kopsia pauciflora & Stem bark & Malaysia & Cytotoxicity \\
\hline 205 & Paucidactine E & Paucidactine & Kopsia pauciflora & Stem bark & Malaysia & Cytotoxicity \\
\hline 206 & Paucidisine & Kopsine & Kopsia pauciflora & Stem bark & Malaysia & Cytotoxicity \\
\hline 207 & (-)-19-Oxoisoeburnamine & Eburnane & Kopsia pauciflora & Stem bark & Malaysia & Cytotoxicity \\
\hline 208 & $(-)-19(R)$-Hydroxyeburnamenine & Eburnane & Kopsia pauciflora & Stem bark & Malaysia & Cytotoxicity \\
\hline 210 & Larutienine B & Eburnane & Kopsia pauciflora & Stem bark & Malaysia & Cytotoxicity \\
\hline 211 & Paucidactine A & Paucidactine & Kopsia pauciflora & Stem bark & Malaysia & Cytotoxicity \\
\hline 212 & Paucidactine B & Paucidactine & Kopsia pauciflora & Stem bark & Malaysia & Cytotoxicity \\
\hline 213 & Paucidactine $\mathrm{C}$ & Paucidactine & Kopsia pauciflora & Stem bark & Malaysia & Cytotoxicity \\
\hline 214 & 5, 22-Dioxokopsane & Kopsine & Kopsia pauciflora & Stem bark & Malaysia & Cytotoxicity \\
\hline 215 & (+)-Eburnamonine & Eburnane & Kopsia pauciflora & Stem bark & Malaysia & Cytotoxicity, \\
\hline 216 & Eburnamenine & Eburnane & Kopsia pauciflora & Stem bark & Malaysia & Cytotoxicity \\
\hline 217 & (-)-Eburnamine & Eburnane & Kopsia pauciflora & Stem bark & Malaysia & Cytotoxicity \\
\hline 218 & (+)-Isoeburnamine & Eburnane & Kopsia pauciflora & Stem bark & Malaysia & Cytotoxicity \\
\hline 219 & (+)-19-Oxoeburnamine & Eburnane & Kopsia pauciflora & Stem bark & Malaysia & Cytotoxicity \\
\hline 220 & $(-)-19(R)$-Hydroxyisoeburnamine & Eburnane & Kopsia pauciflora & Stem bark & Malaysia & Cytotoxicity \\
\hline
\end{tabular}


Table 1. Cont.

\begin{tabular}{|c|c|c|c|c|c|c|}
\hline Comp No & Compound Name & Class Type & Source & Part & Country & Activities \\
\hline 221 & (+)-19(R)-Hydroxyeburnamine & Eburnane & Kopsia pauciflora & Stem bark & Malaysia & Cytotoxicity \\
\hline 222 & Larutienine A & Eburnane & Kopsia pauciflora & Stem bark & Malaysia & Cytotoxicity \\
\hline 223 & $(-)$-Norpleiomutine & Eburnane- Aspidofractinine & Kopsia pauciflora & Stem bark & Malaysia & Cytotoxicity \\
\hline 224 & $(+)$-Kopsoffinol & Eburnane- Aspidofractinine & Kopsia pauciflora & Stem bark & Malaysia & Cytotoxicity \\
\hline 225 & (-)-Demethylnorpleiomutine & Eburnane- Aspidofractinine & Kopsia pauciflora & Stem bark & Malaysia & Cytotoxicity \\
\hline 226 & $(+)$-Kopsoffine & Vincamine- Aspidofractinine & Kopsia pauciflora & Stem bark & Malaysia & Cytotoxicity \\
\hline 228 & Arborisidine & Pericine & Kopsia arborea & Whole plant & Malayan & Cytotoxicity \\
\hline 229 & Arbornamine & Arbornane & Kopsia arborea & Whole plant & Malayan & Cytotoxicity \\
\hline 230 & Kopsinidine C & Kopsine & Kopsia officinalis\$ & Twigs and leaves & China & $\begin{array}{c}\text { Immunosuppressive } \\
\text { activity }\end{array}$ \\
\hline 231 & Kopsinidine D & Kopsine & Kopsia officinalis\$ & Twigs and leaves & China & $\begin{array}{c}\text { Immunosuppressive } \\
\text { activity }\end{array}$ \\
\hline 232 & Kopsinidine E & Kopsine & Kopsia officinalis\$ & Twigs and leaves & China & $\begin{array}{l}\text { Immunosuppressive } \\
\text { activity }\end{array}$ \\
\hline 233 & 11,12-Methylenedioxychanofruticosinic Acid & Methyl Chanofruticosinate & Kopsia officinalis & Twigs and leaves & China & $\begin{array}{c}\text { Immunosuppressive } \\
\text { activity }\end{array}$ \\
\hline 234 & 12-Methoxychanofruticosinic Acid & Methyl Chanofruticosinate & Kopsia officinalis & Twigs and leaves & China & $\begin{array}{c}\text { Immunosuppressive } \\
\text { activity }\end{array}$ \\
\hline 235 & $N_{(4)}$-Methylkopsininate & Aspidofractinine & Kopsia officinalis & Twigs and leaves & China & $\begin{array}{c}\text { Immunosuppressive } \\
\text { activity }\end{array}$ \\
\hline 236 & Chanofruticosinic Acid & Methyl Chanofruticosinate & Kopsia officinalis & Twigs and leaves & China & $\begin{array}{c}\text { Immunosuppressive } \\
\text { activity }\end{array}$ \\
\hline 237 & Kopsinine Methochloride & Aspidofractinine & Kopsia officinalis & Twigs and leaves & China & $\begin{array}{c}\text { Immunosuppressive } \\
\text { activity }\end{array}$ \\
\hline 238 & Demethoxycarbonylkopsin & Kopsine & Kopsia officinalis & Twigs and leaves & China & $\begin{array}{c}\text { Immunosuppressive } \\
\text { activity }\end{array}$ \\
\hline
\end{tabular}


Table 1. Cont.

\begin{tabular}{|c|c|c|c|c|c|c|}
\hline Comp No & Compound Name & Class Type & Source & Part & Country & Activities \\
\hline 239 & Methyl Chanofruticosinate & Methyl Chanofruticosinate & Kopsia officinalis & Twigs and leaves & China & $\begin{array}{l}\text { Immunosuppressive } \\
\text { activity }\end{array}$ \\
\hline 240 & $\begin{array}{c}\text { Methyl } \\
\text { 11,12-Methylenedioxychanofruticosinate }\end{array}$ & Methyl Chanofruticosinate & Kopsia officinalis & Twigs and leaves & China & $\begin{array}{c}\text { Immunosuppressive } \\
\text { activity }\end{array}$ \\
\hline 241 & Methyl 12-Methoxychanofruticosinate & Methyl Chanofruticosinate & Kopsia officinalis & Twigs and leaves & China & $\begin{array}{l}\text { Immunosuppressive } \\
\text { activity }\end{array}$ \\
\hline 242 & $\begin{array}{l}\text { Methyl 11,12-Methylenedioxy- } \mathrm{N}_{1^{-}} \\
\text {Decarbomethoxychanofruticosinate }\end{array}$ & Methyl Chanofruticosinate & Kopsia officinalis & Twigs and leaves & China & $\begin{array}{l}\text { Immunosuppressive } \\
\text { activity }\end{array}$ \\
\hline 243 & Kopsininic Acid & Aspidofractinine & Kopsia officinalis & Twigs and leaves & China & $\begin{array}{l}\text { Immunosuppressive } \\
\text { activity }\end{array}$ \\
\hline 244 & (-)-11,12-Methylenedioxykopsinaline & Aspidofractinine & Kopsia officinalis & Twigs and leaves & China & $\begin{array}{l}\text { Immunosuppressive } \\
\text { activity }\end{array}$ \\
\hline 245 & $\begin{array}{l}\text { (-)-N-Methoxycarbonyl-11,12- } \\
\text { Methylenedioxykopsinaline }\end{array}$ & Aspidofractinine & Kopsia officinalis & Twigs and leaves & China & $\begin{array}{l}\text { Immunosuppressive } \\
\text { activity }\end{array}$ \\
\hline 246 & $\begin{array}{l}\text { (-)-N-Methoxycarbonyl- } \\
\text { 12-Methoxykopsinaline }\end{array}$ & Aspidofractinine & Kopsia officinalis & Twigs and leaves & China & $\begin{array}{l}\text { Immunosuppressive } \\
\text { activity }\end{array}$ \\
\hline 247 & $\begin{array}{l}\text { N-Carbomethoxy-11-Hydroxy-12- } \\
\text { Methoxykopsinaline }\end{array}$ & Aspidofractinine & Kopsia officinalis & Twigs and leaves & China & $\begin{array}{l}\text { Immunosuppressive } \\
\text { activity }\end{array}$ \\
\hline 248 & Kopsinoline & Aspidofractinine & Kopsia officinalis & Twigs and leaves & China & $\begin{array}{l}\text { Immunosuppressive } \\
\text { activity }\end{array}$ \\
\hline 249 & (-)-12-Methoxykopsinaline & Aspidofractinine & Kopsia officinalis & Twigs and leaves & China & $\begin{array}{l}\text { Immunosuppressive } \\
\text { activity }\end{array}$ \\
\hline 250 & 11,12-Methylenedioxykopsinaline $N_{(4)}$ - Oxide & Aspidofractinine & Kopsia officinalis & Twigs and leaves & China & $\begin{array}{l}\text { Immunosuppressive } \\
\text { activity }\end{array}$ \\
\hline 251 & Kopsinine B & Aspidofractinine & Kopsia officinalis & Twigs and leaves & China & $\begin{array}{l}\text { Immunosuppressive } \\
\text { activity }\end{array}$ \\
\hline 252 & Rhazinilam & Aspidosperma & Kopsia officinalis & Twigs and leaves & China & $\begin{array}{l}\text { Immunosuppressive } \\
\text { activity }\end{array}$ \\
\hline 253 & Pleiocarpamine Methochloride & Corynanthe & Kopsia officinalis & Twigs and leaves & China & $\begin{array}{l}\text { Immunosuppressive } \\
\text { activity }\end{array}$ \\
\hline
\end{tabular}


Table 1. Cont.

\begin{tabular}{|c|c|c|c|c|c|c|}
\hline Comp No & Compound Name & Class Type & Source & Part & Country & Activities \\
\hline 254 & Kopsioffine A & Aspidosperma & Kopsia officinalis & Leaves and stems & China & $\begin{array}{l}\text { Yeast } \alpha \text {-glucosidase } \\
\text { inhibitory }\end{array}$ \\
\hline 255 & Kopsioffine B & Aspidosperma & Kopsia officinalis & Leaves and stems & China & $\begin{array}{l}\text { Yeast } \alpha \text {-glucosidase } \\
\text { inhibitory }\end{array}$ \\
\hline 256 & Kopsioffine C & Aspidosperma & Kopsia officinalis & Leaves and stems & China & $\begin{array}{l}\text { Yeast } \alpha \text {-glucosidase } \\
\text { inhibitory }\end{array}$ \\
\hline 257 & Kopsifoline G & Aspidosperma & Kopsia fruticose & $\begin{array}{l}\text { Aerial } \\
\text { parts }\end{array}$ & China & $\begin{array}{c}\text { Cytotoxicity, Antifungal } \\
\text { Antibacterial }\end{array}$ \\
\hline 258 & Kopsifoline H & Aspidosperma & Kopsia fruticose & $\begin{array}{l}\text { Aerial } \\
\text { parts }\end{array}$ & China & $\begin{array}{c}\text { Cytotoxicity, Antifungal, } \\
\text { Antibacterial }\end{array}$ \\
\hline 259 & Kopsifoline I & Aspidosperma & Kopsia fruticose & $\begin{array}{l}\text { Aerial } \\
\text { parts }\end{array}$ & China & $\begin{array}{c}\text { Cytotoxicity, Antifungal, } \\
\text { Antibacterial }\end{array}$ \\
\hline 260 & Kopsifoline J & Aspidosperma & Kopsia fruticose & $\begin{array}{l}\text { Aerial } \\
\text { parts }\end{array}$ & China & $\begin{array}{c}\text { Cytotoxicity, Antifungal, } \\
\text { Antibacterial }\end{array}$ \\
\hline 261 & Kopsifoline K & Aspidosperma & Kopsia fruticose & $\begin{array}{l}\text { Aerial } \\
\text { parts }\end{array}$ & China & $\begin{array}{c}\text { Cytotoxicity, Antifungal, } \\
\text { Antibacterial }\end{array}$ \\
\hline 262 & Kopsifoline A & Aspidosperma & Kopsia fruticose & $\begin{array}{l}\text { Aerial } \\
\text { parts }\end{array}$ & China & $\begin{array}{c}\text { Cytotoxicity, Antifungal } \\
\text { Antibacterial }\end{array}$ \\
\hline 263 & Kopsiarborine A & Aspidofractinine & Kopsia arborea & Aerial parts & China & Cytotoxicity \\
\hline 264 & Kopsiarborine B & Methyl Chanofruticosinate & Kopsia arborea & $\begin{array}{l}\text { Aerial } \\
\text { parts }\end{array}$ & China & Cytotoxicity \\
\hline 265 & Kopsiarborine C & Aspidosperma & Kopsia arborea & Aerial parts & China & Cytotoxicity \\
\hline 266 & Kopsiaofficine A & Aspidofractinine & Kopsia officinalis & Aerial parts & China & Cytotoxicity \\
\hline 267 & Kopsiaofficine B & Paucidactine & Kopsia officinalis & Aerial parts & China & Cytotoxicity \\
\hline 268 & Kopsiaofficine C & Aspidofractinine & Kopsia officinalis & Aerial parts & China & Cytotoxicity \\
\hline 269 & Kopsiofficine $\mathrm{H}$ & Eburnane & Kopsia officinalis & Stems & China & Anti-inflammatory \\
\hline 270 & Kopsiofficine I & Eburnane & Kopsia officinalis & Stems & China & Anti-inflammatory \\
\hline 271 & Kopsiofficine J & Eburnane & Kopsia officinalis & Stems & China & Anti-inflammatory \\
\hline
\end{tabular}


Table 1. Cont.

\begin{tabular}{|c|c|c|c|c|c|c|}
\hline Comp No & Compound Name & Class Type & Source & Part & Country & Activities \\
\hline 272 & Kopsiofficine K & Eburnane & Kopsia officinalis & Stems & China & Anti-inflammatory \\
\hline 273 & Kopsiofficine L & Kopsine & Kopsia officinalis & Stems & China & Anti-inflammatory \\
\hline 274 & (+)-O-Methyleburnamine & Eburnane & Kopsia officinalis & Stems & China & Anti-inflammatory \\
\hline 275 & (-)-O-Methylisoeburnamine & Eburnane & Kopsia officinalis & Stems & China & Anti-inflammatory \\
\hline 276 & 16-Isoeburnamine & Eburnane & Kopsia officinalis & Stems & China & Anti-inflammatory \\
\hline 277 & 20-Oxoeburnamenine & Eburnane & Kopsia officinalis & Stems & China & Anti-inflammatory \\
\hline 279 & $\begin{array}{c}\text { Methyl } \\
\mathrm{N} \text {-(Decarbomethoxy)-11,12-(Methylenedioxy) } \\
\text { Chanofruticosinate }\end{array}$ & Methyl Chanofruticosinate & Kopsia officinalis & Stems & China & Anti-inflammatory \\
\hline 280 & O-Methylleuconolam & Aspidosperma & Kopsia officinalis & Stems & China & Anti-inflammatory \\
\hline 281 & Leuconodine D & Aspidosperma & Kopsia officinalis & Stems & China & Anti-inflammatory \\
\hline 282 & Oxayohimban-16-Carboxylic Acid & Corynanthe & Kopsia officinalis & Stems & China & Anti-inflammatory \\
\hline 283 & 19, 20-Dihydroisositsirikine & Corynanthe & Kopsia officinalis & Stems & China & Anti-inflammatory \\
\hline 285 & Rauvomine B & Sarpagine & Rauvolfia vomitoria & Aerial parts & China & Anti-inflammatory \\
\hline 286 & Peraksine & Sarpagine & Rauvolfia vomitoria & Aerial parts & China & Anti-inflammatory \\
\hline 287 & Alstoyunine A & Sarpagine & Rauvolfia vomitoria & Aerial parts & China & Anti-inflammatory \\
\hline 288 & 11-Hydroxyburnamine & Picraline & Rauvolfia yunnanensis & Whole plant & China & $\begin{array}{c}\text { Cytotoxicity } \\
\text { Immunosuppressive }\end{array}$ \\
\hline 289 & Rauvoyunnanine A & Sarpagine & Rauvolfia yunnanensis & Whole plant & China & $\begin{array}{c}\text { Cytotoxicity } \\
\text { Immunosuppressive }\end{array}$ \\
\hline 290 & Rauvoyunnanine B & Corynanthe & Rauvolfia yunnanensis & Whole plant & China & $\begin{array}{c}\text { Cytotoxicity } \\
\text { Immunosuppressive }\end{array}$ \\
\hline 291 & Lochnerine & Sarpagine & Rauvolfia yunnanensis & Whole plant & China & $\begin{array}{c}\text { Cytotoxicity } \\
\text { Immunosuppressive }\end{array}$ \\
\hline
\end{tabular}


Table 1. Cont.

\begin{tabular}{|c|c|c|c|c|c|c|}
\hline Comp No & Compound Name & Class Type & Source & Part & Country & Activities \\
\hline 292 & Serpentinic Acid & Corynanthe & Rauvolfia yunnanensis & Whole plant & China & $\begin{array}{c}\text { Cytotoxicity } \\
\text { Immunosuppressive }\end{array}$ \\
\hline 293 & Reserpine & Corynanthe & Rauvolfia yunnanensis & Whole plant & China & $\begin{array}{c}\text { Cytotoxicity } \\
\text { Immunosuppressive }\end{array}$ \\
\hline 294 & (-)-Yohimbine & Corynanthe & Rauvolfia yunnanensis & Whole plant & China & $\begin{array}{c}\text { Cytotoxicity } \\
\text { Immunosuppressive }\end{array}$ \\
\hline 295 & Ajmaline & Ajmaline & Rauvolfia yunnanensis & Whole plant & China & $\begin{array}{c}\text { Cytotoxicity } \\
\text { Immunosuppressive }\end{array}$ \\
\hline 297 & Ajmalicine & Corynanthe & Rauvolfia yunnanensis & Whole plant & China & $\begin{array}{c}\text { Cytotoxicity } \\
\text { Immunosuppressive }\end{array}$ \\
\hline 298 & Sitsirikine & Corynanthe & Rauvolfia yunnanensis & Whole plant & China & $\begin{array}{c}\text { Cytotoxicity } \\
\text { Immunosuppressive }\end{array}$ \\
\hline 299 & Strictosidinic Acid & Strictosidine & Rauvolfia yunnanensis & Whole plant & China & $\begin{array}{c}\text { Cytotoxicity } \\
\text { Immunosuppressive }\end{array}$ \\
\hline 300 & Caboxine B & Corynanthe & Rauvolfia yunnanensis & Whole plant & China & $\begin{array}{c}\text { Cytotoxicity } \\
\text { Immunosuppressive }\end{array}$ \\
\hline 301 & Isocaboxine B & Corynanthe & Rauvolfia yunnanensis & Whole plant & China & $\begin{array}{c}\text { Cytotoxicity } \\
\text { Immunosuppressive }\end{array}$ \\
\hline 302 & Spegatrine & Sarpagine & Rauvolfia yunnanensis & Whole plant & China & $\begin{array}{c}\text { Cytotoxicity } \\
\text { Immunosuppressive }\end{array}$ \\
\hline 303 & 19(S),20(R)-Dihydroperaksine & Sarpagine & Rauvolfia yunnanensis & Whole plant & China & $\begin{array}{c}\text { Cytotoxicity } \\
\text { Immunosuppressive }\end{array}$ \\
\hline 304 & Ervataine & Iboga & Ervatamia yunnanensis & Stems & China & $\_\$$ \\
\hline 305 & Ibogaine & Iboga & Ervatamia yunnanensis & Stems & China & $\_\$$ \\
\hline 306 & Coronaridine & Iboga & Ervatamia yunnanensis & Stems & China & $\begin{array}{l}\text { Acetylcholinesterase } \\
\text { Inhibition }\end{array}$ \\
\hline 307 & Heyneanine & Iboga & Ervatamia yunnanensis & Stems & China & - \\
\hline 308 & Voacangine Hydroxyindolenine & Iboga & Ervatamia yunnanensis & Stems & China & _- \\
\hline
\end{tabular}


Table 1. Cont.

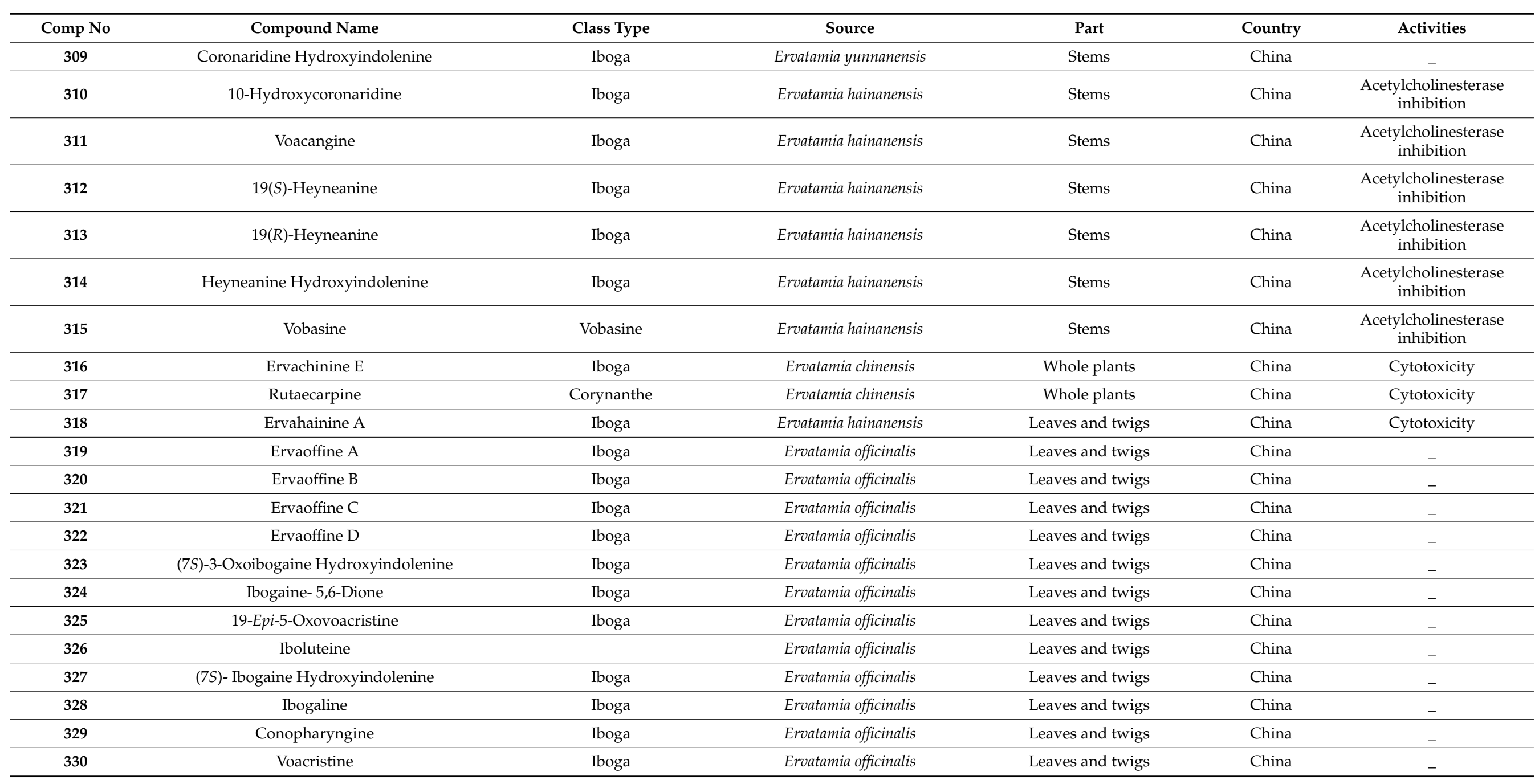


Table 1. Cont.

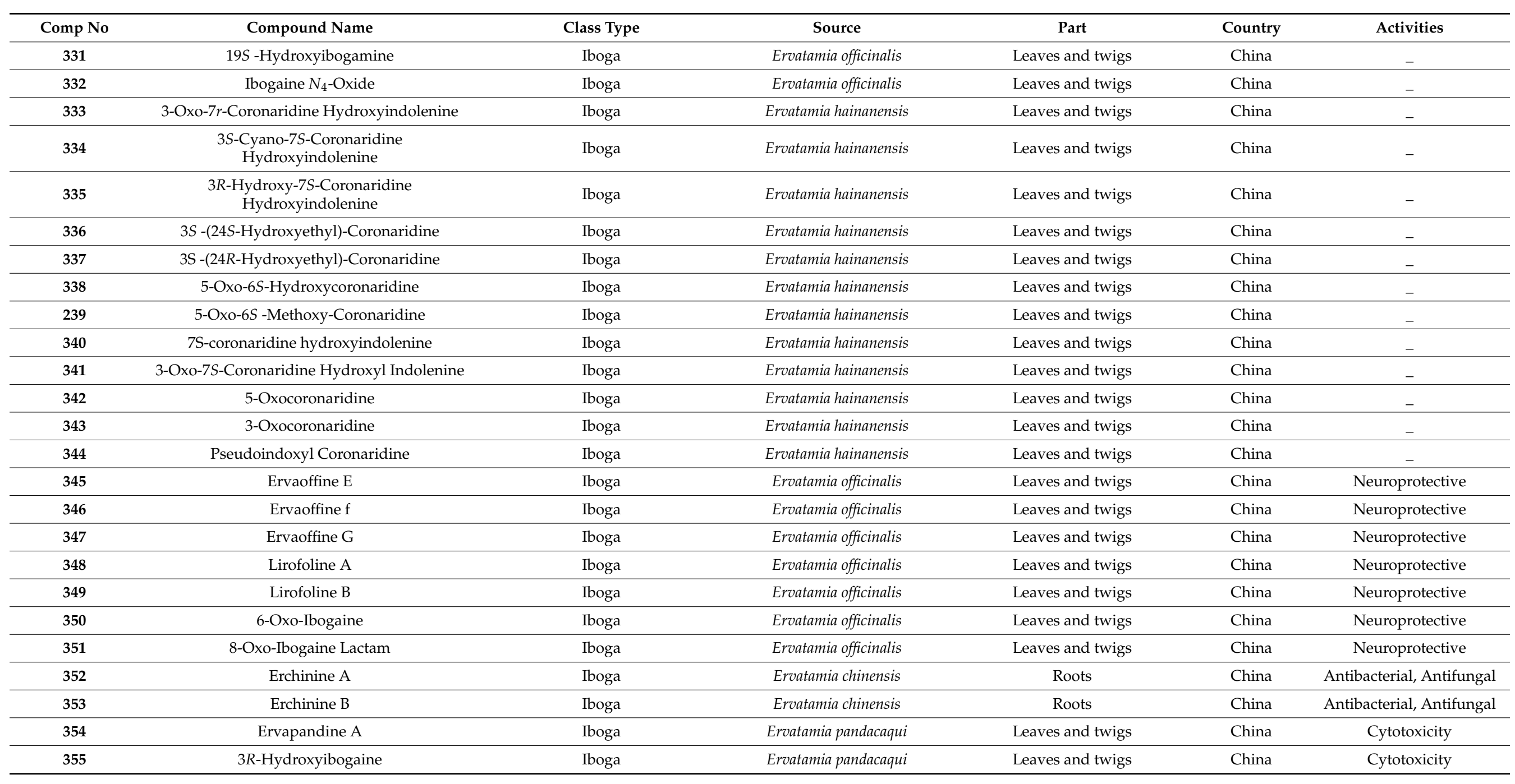


Table 1. Cont.

\begin{tabular}{|c|c|c|c|c|c|c|}
\hline Comp No & Compound Name & Class Type & Source & Part & Country & Activities \\
\hline 356 & 12-Hydroxyakuammicine $N_{4}$-Oxide & Akuammicine & Ervatamia pandacaqui & Leaves and twigs & China & Cytotoxicity \\
\hline 357 & 19-Epi-Voacristine & Iboga & Ervatamia pandacaqui & Leaves and twigs & China & Cytotoxicity \\
\hline 358 & Taberdivarine I & Iboga & Ervatamia pandacaqui & Leaves and twigs & China & Cytotoxicity \\
\hline 359 & 12-Hydroxyakuamicine & Akuammicine & Ervatamia pandacaqui & Leaves and twigs & China & Cytotoxicity \\
\hline 360 & Ervadivamine A & Vobasine-Iboga-Vobasine & Ervatamia divaricate & Roots & China & Cytotoxicity \\
\hline 361 & Ervadivamine B & Vobasine-Iboga-Vobasine & Ervatamia divaricate & Roots & China & Cytotoxicity \\
\hline 363 & Ibogamine & Iboga & Ervatamia divaricate & Roots & China & Cytotoxicity \\
\hline 364 & Ervatamine & Flabelliformide & Ervatamia yunnanensis & Stems & China & - \\
\hline 365 & 20-Epi-Ervatamine & Flabelliformide & Ervatamia yunnanensis & Stems & China & - \\
\hline 366 & Dregamine & Vobasine & Ervatamia yunnanensis & Stems & China & - \\
\hline 367 & Tabernaemontanine & Vobasine & Ervatamia yunnanensis & Stems & China & - \\
\hline 368 & Apparicine & Iboga & Ervatamia yunnanensis & Stems & China & - \\
\hline 369 & Isovoacangine & Apparicine & Ervatamia yunnanensis & Stems & China & - \\
\hline 371 & Conodusine B & Iboga & Tabernaemontana corymbosa & Stem bark & Malaysia & Cytotoxicity \\
\hline 372 & Conodusine C & Iboga & Tabernaemontana corymbosa & Stem bark & Malaysia & Cytotoxicity \\
\hline 373 & Conodusine D & Iboga & Tabernaemontana corymbosa & Stem bark & Malaysia & Cytotoxicity \\
\hline 374 & Conodusine E & Iboga & Tabernaemontana corymbosa & Stem bark & Malaysia & Cytotoxicity \\
\hline 375 & Apocidine A & Aspidosperma & Tabernaemontana corymbosa & Stem bark & Malaysia & Cytotoxicity \\
\hline 376 & Apocidine B & Aspidosperma & Tabernaemontana corymbosa & Stem bark & Malaysia & Cytotoxicity \\
\hline 377 & Conoduzidine A & Vincamine & Tabernaemontana corymbosa & Stem bark & Malaysia & Cytotoxicity \\
\hline 378 & Tabernamidine A & Vobasine-Iboga & Tabernaemontana corymbosa & Stem bark & Malaysia & Cytotoxicity \\
\hline 379 & Tabernamidine B & Vobasine-Iboga & Tabernaemontana corymbosa & Stem bark & Malaysia & Cytotoxicity \\
\hline 380 & (+)-Catharanthine & Iboga & Tabernaemontana corymbosa & Stem bark & Malaysia & Cytotoxicity \\
\hline
\end{tabular}


Table 1. Cont.

\begin{tabular}{|c|c|c|c|c|c|c|}
\hline Comp No & Compound Name & Class Type & Source & Part & Country & Activities \\
\hline 381 & Tabernamine & Vobasine-Iboga & Tabernaemontana corymbosa & Stem bark & Malaysia & Cytotoxicity \\
\hline 382 & $19^{\prime}(S)$-Hydroxytabernamine & Vobasine-Iboga & Tabernaemontana corymbosa & Stem bark & Malaysia & Cytotoxicity \\
\hline 383 & $19^{\prime}(R)$-Hydroxytabernamine & Vobasine-Iboga & Tabernaemontana corymbosa & Stem bark & Malaysia & Cytotoxicity \\
\hline 384 & 16'-Decarbomethoxyvoacamine & Vobasine-Iboga & Tabernaemontana corymbosa & Stem bark & Malaysia & Cytotoxicity \\
\hline 385 & Isoakuammiline & Corynanthe & Tabernaemontana litoralis & Fruits & USA & - \\
\hline 386 & 18-Hydroxypseudovincadifformine & Iboga & Tabernaemontana litoralis & Fruits & USA & - \\
\hline 388 & Strictosidine & Strictosidine & Tabernaemontana litoralis & Fruits & USA & - \\
\hline 389 & \$Tabervarine A & Iboga & Tabernaemontana divaricate & Leaves and twigs & China & Cytotoxicity \\
\hline 390 & \$Tabervarine B & Iboga & Tabernaemontana divaricate & Leaves and twigs & China & Cytotoxicity \\
\hline 391 & Vobasidine C & Vobasine & Tabernaemontana divaricate & Leaves and twigs & China & Cytotoxicity \\
\hline 392 & Ervadivaricatine B & Vobasine-Iboga & Tabernaemontana divaricate & Leaves and twigs & China & Cytotoxicity \\
\hline 393 & Pedunculine & Aspidosperma- Aspidosperma & Tabernaemontana divaricate & Leaves and twigs & China & Cytotoxicity \\
\hline 394 & Polyervine & Aspidosperma- Aspidosperma & Tabernaemontana divaricate & Leaves and twigs & China & Cytotoxicity \\
\hline 396 & 19,20-Dihydrovobparicine & Vobasine-Apparicine & Tabernaemontana divaricate & Stems & China & Cytotoxicity \\
\hline 397 & 10'- Demethoxy-19,20-Dihydrovobatensine D & Vobasine-Iboga & Tabernaemontana divaricate & Stems & China & Cytotoxicity \\
\hline 398 & 3'-(2-Oxopropyl)Ervahanine A & Sarpagine-Iboga & Tabernaemontana divaricate & Stems & China & Cytotoxicity \\
\hline 399 & Ervahanine A & Sarpagine-Iboga & Tabernaemontana divaricate & Stems & China & Cytotoxicity \\
\hline 400 & Vobparicine & Vobasine-Apparicine & Tabernaemontana divaricate & Stems & China & Cytotoxicity \\
\hline 401 & 19,20-Dihydrotabernamine & Vobasine-Iboga & Tabernaemontana divaricate & Stems & China & Cytotoxicity \\
\hline 402 & 19,20-Dihydrotabernamine A & Vobasine-Iboga & Tabernaemontana divaricate & Stems & China & Cytotoxicity \\
\hline 403 & Taberdivarines E & Vobasine-Iboga & Tabernaemontana divaricate & Stems & China & Cytotoxicity \\
\hline 404 & Tubotaiwine & Strychnos & Tabernaemontana divaricate & Stems & China & Cytotoxicity \\
\hline 405 & $\begin{array}{l}\text { Hydroxy-3-(2-Oxopropyl) Coronaridine } \\
\text { Indolenine }\end{array}$ & Iboga & Tabernaemontana divaricate & Stems & China & Cytotoxicity \\
\hline
\end{tabular}


Table 1. Cont.

\begin{tabular}{|c|c|c|c|c|c|c|}
\hline Comp No & Compound Name & Class Type & Source & Part & Country & Activities \\
\hline 406 & Deoxytubulosine & Corynanthe bisindole & Tabernaemontana divaricate & Stems & China & Cytotoxicity \\
\hline 407 & $\begin{array}{l}(3 R, 7 S, 14 R, 19 S, 20 R)-19- \\
\text { Hydroxypseudovincadifformine }\end{array}$ & Iboga & Tabernaemontana bufalina & Branches and leaves & China & Cytotoxicity \\
\hline 408 & 17-Demethoxy-Hydroisorhyn Chophylline & Corynanthe & Tabernaemontana bufalina & Branches and leaves & China & Cytotoxicity \\
\hline 409 & 17-Demethoxy-Isorhynchophylline & Corynanthe & Tabernaemontana bufalina & Branches and leaves & China & Cytotoxicity \\
\hline 410 & Voachalotine & Akuammidine & Tabernaemontana bufalina & Branches and leaves & China & Cytotoxicity \\
\hline 411 & 12-Methoxyl-Voaphylline & Aspidosperma & Tabernaemontana bufalina & Branches and leaves & China & Cytotoxicity \\
\hline 412 & Conophylline & Aspidosperma- Aspidosperma & Tabernaemontana bufalina & Branches and leaves & China & Cytotoxicity \\
\hline 413 & 5,6-Dioxo-11-Methoxy Voacangine & Iboga & Tabernaemontana contorta & Fruits & Cameroon & Anti-inflammatory \\
\hline 414 & (-)-Apparicin-21-One & Apparicine & Tabernaemontana contorta & Fruits & Cameroon & Anti-inflammatory \\
\hline 415 & Tabernabovine A & Corynanthe bisindole & Tabernaemontana bovina & Leaves & China & Anti-inflammatory \\
\hline 416 & Tabernabovine B & Aspidosperma & Tabernaemontana bovina & Leaves & China & Anti-inflammatory \\
\hline 417 & Tabernabovine $\mathrm{C}$ & Iboga & Tabernaemontana bovina & Leaves & China & Anti-inflammatory \\
\hline 418 & Secopleiocarpamine A & Corynanthe & Rhazya stricta & Aerial parts & Pakistan & Antifungal \\
\hline 420 & $\begin{array}{c}\text { 2-Ethyl-3[2-(3-Ethyl-1,2,3,6- } \\
\text { Tetrahydropyridine)Ethyl]-Indole }\end{array}$ & Secodine & Rhazya stricta & Aerial parts & Pakistan & Antifungal \\
\hline 421 & 2-Ethyl-3[2-(3-Ethylpiperidine)Ethyl]-Indole & Secodine & Rhazya stricta & Aerial parts & Pakistan & Antifungal \\
\hline 422 & Tetrahydrosecodine & Secodine & Rhazya stricta & Aerial parts & Pakistan & Antifungal \\
\hline 423 & 16,17-Dihydrosecodine & Secodine & Rhazya stricta & Aerial parts & Pakistan & Antifungal \\
\hline 424 & Deacetylakuammilin & Akuammiline & Rhazya stricta & Aerial parts & Pakistan & Antifungal \\
\hline 425 & Rhazimal & Akuammiline & Rhazya stricta & Aerial parts & Pakistan & Antifungal \\
\hline 426 & Strictamine- $N$-Oxide & Akuammiline & Rhazya stricta & Aerial parts & Pakistan & Antifungal \\
\hline 427 & Rhazinaline & Akuammiline & Rhazya stricta & Aerial parts & Pakistan & Antifungal \\
\hline 428 & Rhazinaline $N_{\mathrm{b}}$-Oxide & Akuammiline & Rhazya stricta & Aerial parts & Pakistan & Antifungal \\
\hline 429 & Akuammicine & Akummicine & Rhazya stricta & Aerial parts & Pakistan & Antifungal \\
\hline 430 & 16R-E-Isositsirikine & Corynanthe & Rhazya stricta & Aerial parts & Pakistan & Antifungal \\
\hline
\end{tabular}


Table 1. Cont.

\begin{tabular}{|c|c|c|c|c|c|c|}
\hline Comp No & Compound Name & Class Type & Source & Part & Country & Activities \\
\hline 431 & Dihydrositsirikine & Corynanthe & Rhazya stricta & Aerial parts & Pakistan & Antifungal \\
\hline 432 & Antirhine & Corynanthe & Rhazya stricta & Aerial parts & Pakistan & Antifungal \\
\hline 433 & Vincadifformine $N_{(4)}$-Oxide & Aspidosperma & Rhazya stricta & Aerial parts & Pakistan & Antifungal \\
\hline 434 & Eburenine & Aspidosperma & Rhazya stricta & Aerial parts & Pakistan & Antifungal \\
\hline 435 & Winchinine B & Aspidosperma & Rhazya stricta & Aerial parts & Pakistan & Antifungal \\
\hline 436 & Quebrachamine & Aspidosperma & Rhazya stricta & Aerial parts & Pakistan & Antifungal \\
\hline 438 & 16-Epi-Stemmadenine- $\mathrm{N}$-Oxide & Corynanthe & Rhazya stricta & Leaves & Saudi Arabia & Cytotoxicity \\
\hline 439 & Stemmadenine- $N$-Methyl & Corynanthe & Rhazya stricta & Leaves & Saudi Arabia & Cytotoxicity \\
\hline 440 & 20-Epi-Antirhine & Corynanthe & Rhazya stricta & Leaves & Saudi Arabia & Cytotoxicity \\
\hline 441 & Isopicrinine & Picrinine & Rhazya stricta & Leaves & Saudi Arabia & Cytotoxicity \\
\hline 442 & Epirhazyaminine & & Rhazya stricta & Aerial parts & Saudi Arabia & Cytotoxicity \\
\hline 443 & 20-Epi-sitsirikine & & Rhazya stricta & Aerial parts & Saudi Arabia & Cytotoxicity \\
\hline 444 & Strictamine & & Rhazya stricta & Aerial parts & Saudi Arabia & Cytotoxicity \\
\hline
\end{tabular}




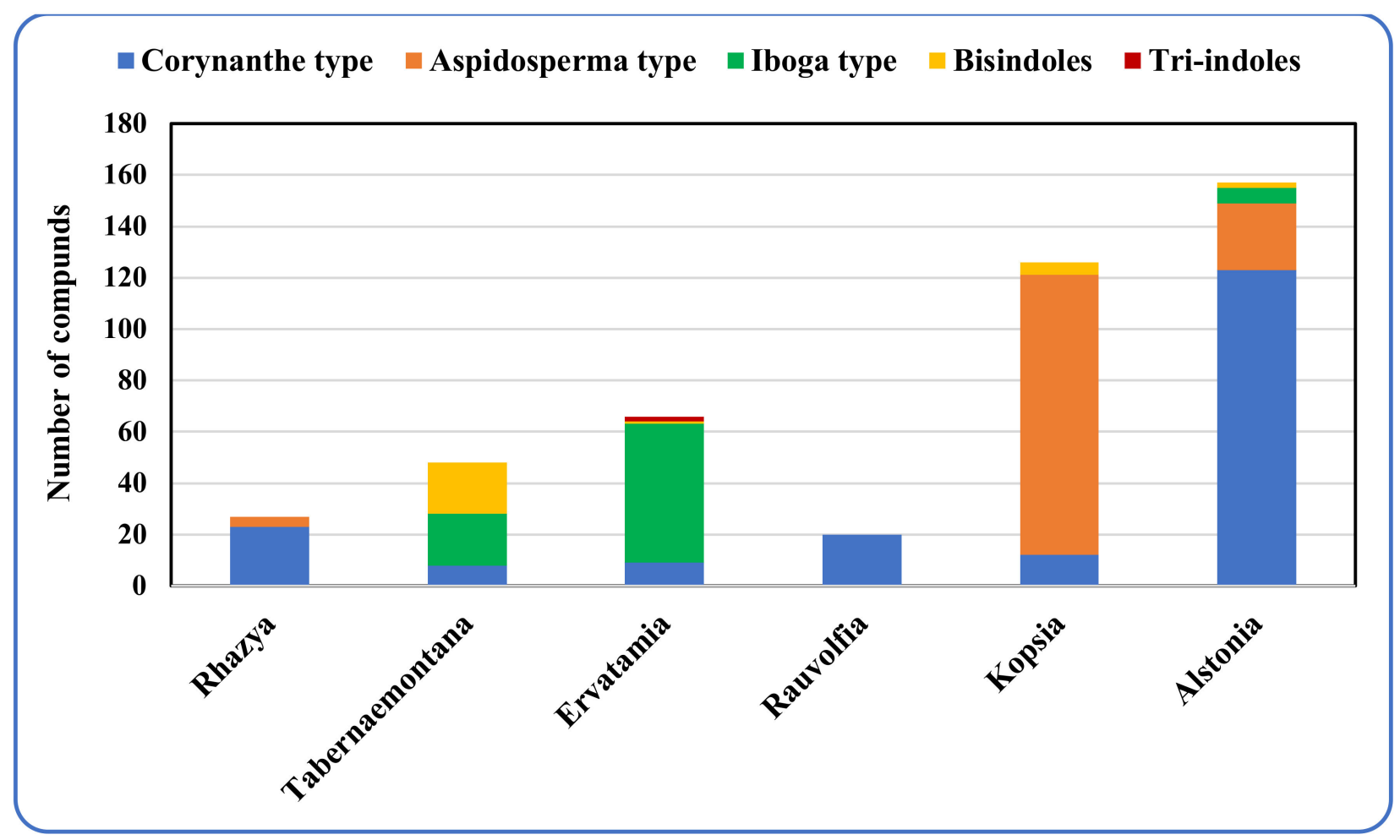

Figure 3. The types of the structures identified monoterpenoid alkaloids from the six genera.

Additionally, the future prospective and emphasizing the research gaps and highlighting the roadmap to discover the potent bioactive monoterpenoid alkaloids, which could be a drug lead from the six genera. Also, this review will discuss the reported structural activity relationships. 


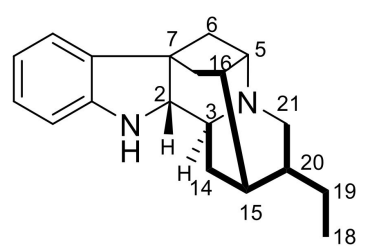

Ajmaline

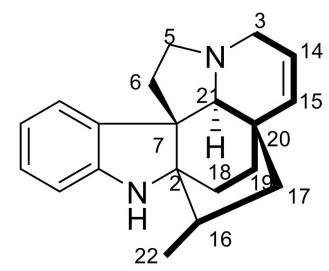

Aspidofractinine

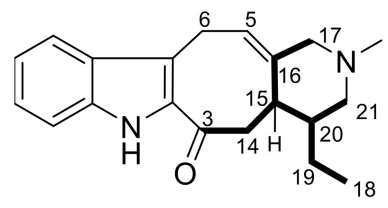

Flabelliformide

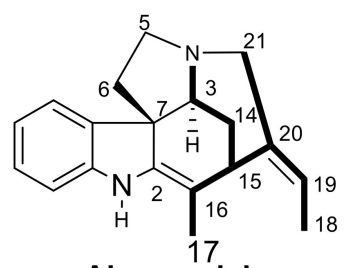

Akuammicine

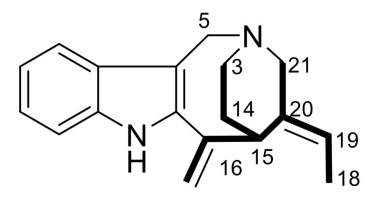

Apparicine

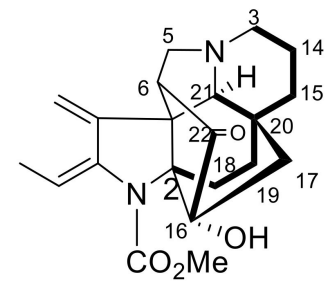

Kopsine
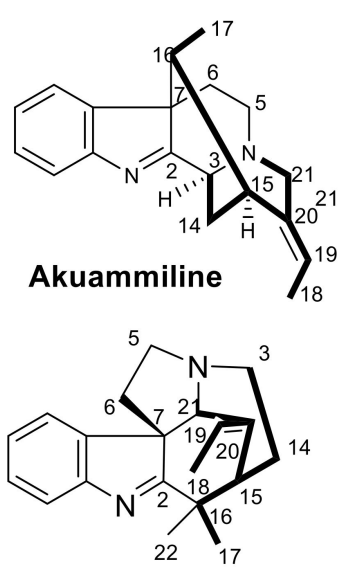

Aspiodspermata

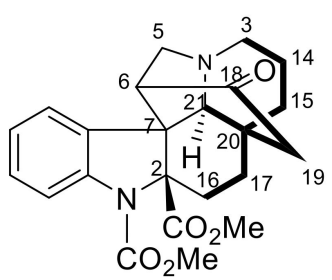

Methyl Chanofruticosinate
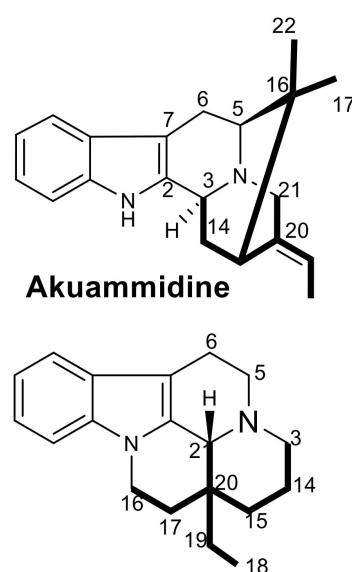

Eburnane

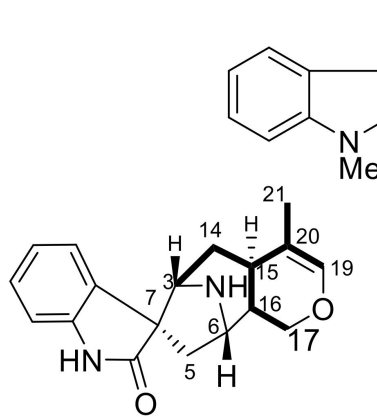

Macroline oxindole

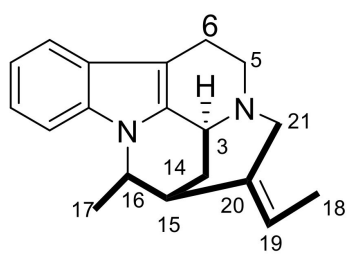

Pleiocarpaman

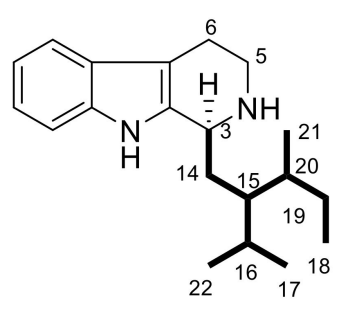

Strictosidine

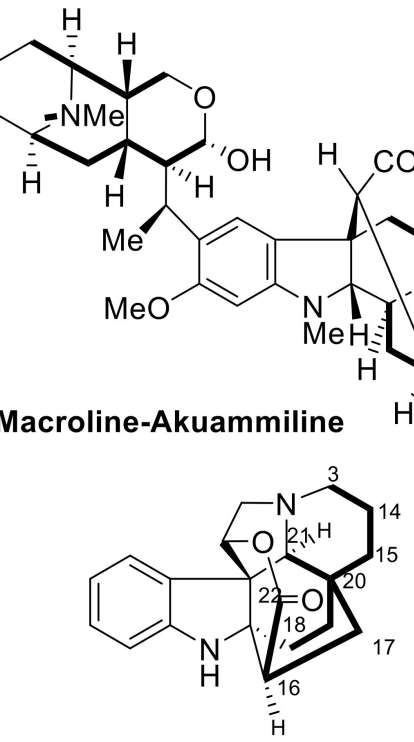

Paucidactine

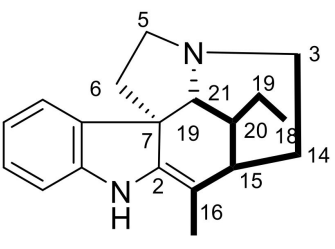

Strychnos

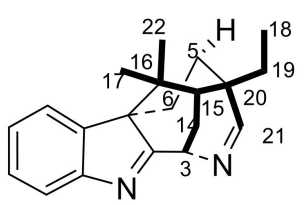

Nareline<smiles>CC=C(C)CNC</smiles>

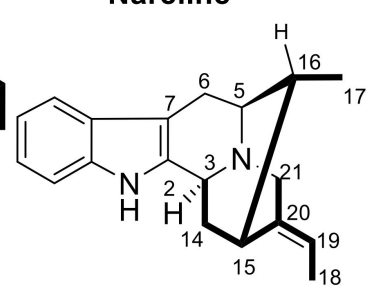<smiles>CC=C1CCCN(CCc2c(C(C)C)[nH]c3ccccc23)C1</smiles>

Secodine
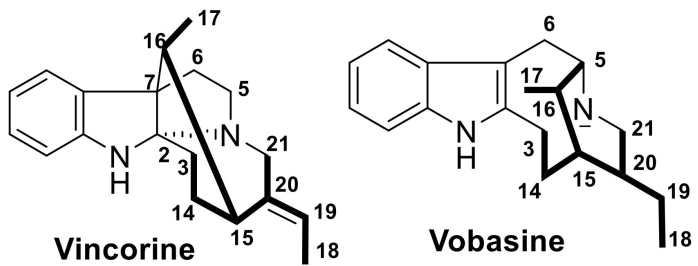

Figure 4. Common monoterpenoid indole alkaloidal skeletons of the six genera. 


\section{Alstonia}

Plants of the genus Alstonia are grown in Africa and Asia. It includes 60 species, which were recognized as rich source of heterocyclic monoterpene indole alkaloids. It has different names according to the geographical sources, including Devil tree, Australian fever bush, dita bark, Australian quinine, fever bark and palimara. Alstonia bark shows potent therapeutic effects including anti-inflammatory, antirheumatic, analgesic, antidiabetic, antimalarial, antipyretic, antihelminthic, antibiotic, antimicrobial, anticancer, antibacterial and antitussive effects [18-20].

Three monoterpene indole alkaloids (MIAs) derivatives, $(14 \alpha, 15 \alpha)-14,15$ - epoxyaspidofractinine (1) and maireines A (2) and B (3) have been isolated from the leaves and twigs of A. mairei [21]. Additionally, venalstonine (4) [22], (-)-minovincinine (5) [23], (-)-11-methoxyminovincinine (6) [24], (-)-echitovenine (7) [25], echitovenaldine (8) [26], echitovenidine (9), 11-methoxyechitovenidine (10) [27], echitoveniline (11), 11- methoxyechitoveniline (12) [24], echitoserpidine (13) [28],11-methoxyechitoserpidine (14) [29], (19S)vindolinine (15) [22], lochnericine (16), tabersonine (17) [30], perakine (18) [31], picrinine (19) [32], F (20) [33], picralinal (21) [34] and rhazimol (22) [35] were isolated from the same species (Figure 5). These compounds were elucidated through the interpretation of different spectroscopic measurements including 1D and 2D NMR and MS. Interesting in compound (1) was the interpretation of the Rotating Frame Overhauser Enhancement Spectroscopy (ROSY) spectrum led to the establishment of the $\alpha$-orientation of the epoxy moiety. Compounds 1-22 were evaluated against five human cancer cells, hepatocellular carcinoma (SMMC-7721), breast (SK-BR-3), pancreatic (PANC-1), human myeloid leukemia (HL-60), and lung (A-549) with $\mathrm{IC}_{50}$ values $>40 \mu \mathrm{M}$ [21].

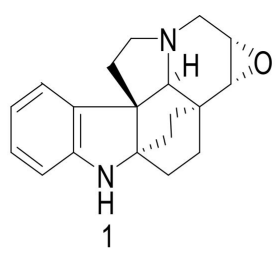<smiles>[R]C(=O)C1=C2Nc3cc([R])ccc3[C@@]2(C)C[C@]2(C([R])C)CCCN3CCC[C@@H]1[C@H]32</smiles><smiles>CCC12CC(C(C)=O)=C3Nc4ccccc4[C@@]34CCN(CC3OC31)[C@@H]24</smiles>

$2 \mathrm{R}_{1}=\mathrm{OMe}, \mathrm{R}_{2}=\mathrm{S}_{4}$ $3 \mathrm{R}_{1}=\mathrm{H}, \mathrm{R}_{2}=\mathrm{S}_{4}$ $5 \mathrm{R}_{1}=\mathrm{H}, \mathrm{R}_{2}=\mathrm{OH}$ $6 \mathrm{R}_{1}=\mathrm{OMe}, \mathrm{R}_{2}=\mathrm{OH}$ $7 \mathrm{R}_{1}=\mathrm{H}, \mathrm{R}_{2}=\mathrm{OAC}$ $8 R_{1}=O M e, R_{2}=O A C$ $9 \mathrm{R}_{1}=\mathrm{H}, \mathrm{R}_{2}=\mathrm{S}_{1}$ $10 R_{1}=O M e, R_{2}=S_{1}$ $11 \mathrm{R}_{1}=\mathrm{H}, \mathrm{R}_{2}=\mathrm{S}_{2}$ $12 R_{1}=O M e, R_{2}=S_{2}$ $13 R_{1}=\mathrm{H}, \mathrm{R}_{2}=\mathrm{S}_{3}$ $14 R_{1}=O M e, R_{2}=S_{3}$

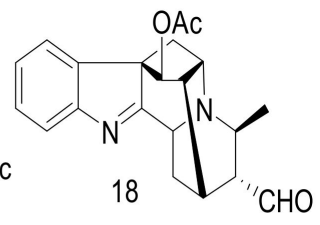

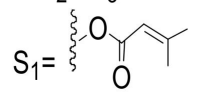<smiles>COc1cc(C(=O)OCC(C)[Se])cc(OC)c1OC</smiles>

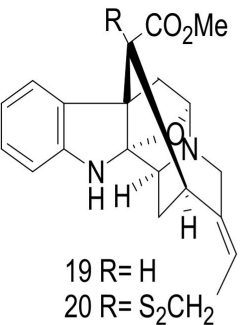

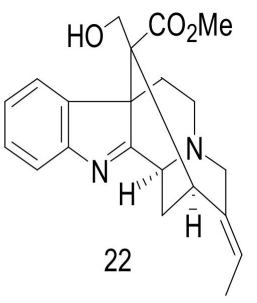
$21 \mathrm{R}=\mathrm{CHO}$<smiles>COC(=O)c1cc(OC)c2c(c1)OCO2</smiles><smiles>COc1cc(/C=C/C(=O)O[Sb])cc(OC)c1</smiles>

Figure 5. Compounds 1-22. 
The majority of reported alkaloids from $A$. scholaris, were of the picrinine type whereas, those isolated from $A$. yunnanensis were either picrinine or aspidospermine types.

Alsmaphorazines A (23) and B (24) (Figure 6) were identified from the leaves of malaysian A. pneumatophore. The chemical structures were determined on the basis of 2D NMR and MS spectral analysis. These compounds had an unprecedented skeleton containing an 1,2-oxazine (six-member ring) and an isoxazolidine (five-member ring) [36]. The absolute configuration of alsmaphorazine B was determined using CD spectral analysis. The absolute configuration of alsmaphorazine B (24) was studied by comparing its experimental CD spectrum with the calculated CD spectrum, with the CD calculations performed by Turbomole 6.1using the Time-Dependent Density Functional Theory (TD-DFT-B3LYP/TZVPP) level of theory on RI-DFTBP386LYP/TZVPP optimized geometries. Compound 23 inhibited the production of nitric oxide (NO) in an LPS-stimulated J774.1 cell with an $\mathrm{IC}_{50}$ value $=49.2 \mu \mathrm{M}$, without affecting the cell viability, whereas compound 24 showed no inhibitory effect at $50.0 \mu \mathrm{M}$. Compound 23 was more potent as an anti-inflammatory agent due to the presence of a hydroxyl group at C-12 [36].

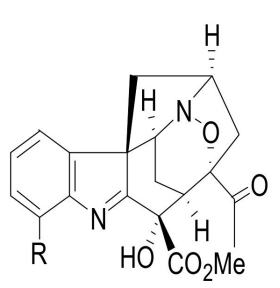

$23 \mathrm{R}=\mathrm{OH}$ $24 \mathrm{R}=\mathrm{H}$

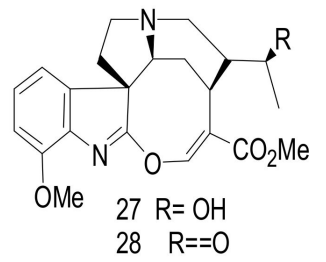

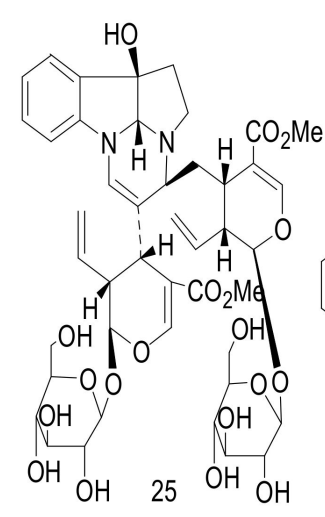

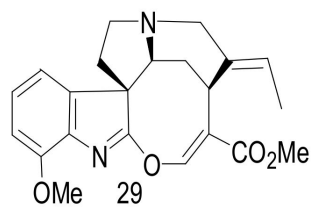

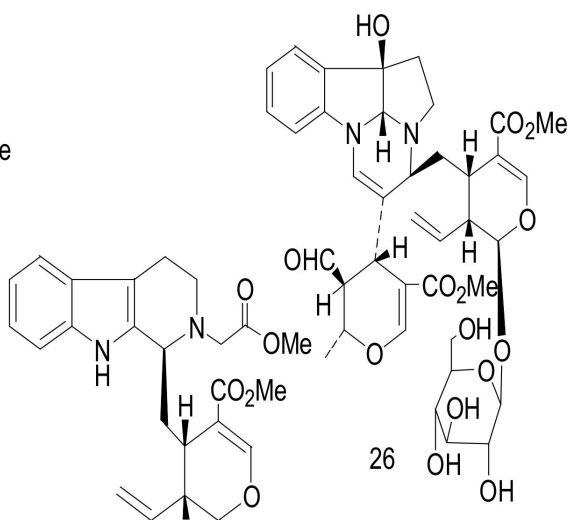

30

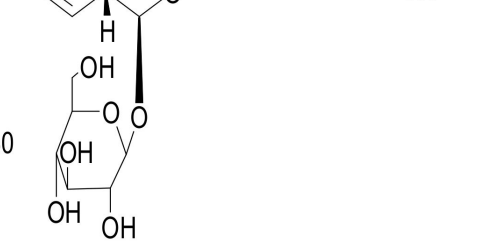

Figure 6. Compounds 23-30.

Alstrostines A (25) and B (26) were determined as derived from the condensation of tryptophan and secologanin in a ratio of 1:2. They were isolated from Alstonia rostrata [37]. The structures were established by measuring ${ }^{1} \mathrm{H},{ }^{13} \mathrm{C}, \mathrm{HSQC}, \mathrm{HMBC},{ }^{1} \mathrm{H}-{ }^{1} \mathrm{H}$ COSY and ROESY. Compounds, 25 and 26, exhibited a weak cytotoxicity against five human cancer cells, hepatocellular carcinoma (SMMC-7721), breast (MCF-7), colon (SW480), myeloid leukemia (HL-60) and lung (A-549), with $\mathrm{IC}_{50}$ values $>40 \mu \mathrm{M}$ [37].

Alstrostines C-F (27-30) (Figure 6) were isolated from the leaves and twigs of Chinese A. rostrata [38]. Compounds 27-30 showed a characteristic UV absorption at 326, 275 and $214 \mathrm{~nm}$, which indicated the presence of an indole alkaloid with a $\beta$-anilineacrylate system. The chemical structure elucidation was confirmed by 1D and 2D NMR. Compounds 2730 showed weak cytotoxicity against five human cancer cells, breast (SK-BR-3), human myeloid leukemia (HL-60), pancreatic (PANC-1), hepatocellular carcinoma (SMMC-7721) and lung (A-549) cells, with $\mathrm{IC}_{50}$ values $>40 \mu \mathrm{M}$ [38].

Five MIAs, 11-hydroxy-6,7-epoxy-8-oxo-vincadifformine (31), 14-chloro-15-hydroxyvinca difformine (32), perakine $\mathrm{N}_{4}$-oxide (33), raucaffrinoline $N_{4}$-oxide (34), and vinorine $N_{1}, N_{4}{ }^{-}$ dioxide (35) (Figure 7) have been reported from A. yunnanensis. Additionally, three compounds, 11-methoxy-6,7-epoxy-8-oxovincadifformine (36), vinorine N4-oxide (37) and vinorine (38) have also been found from the same plant [39]. The chemical structures 
were established based on 1D and 2D $\left({ }^{1} \mathrm{H}-{ }^{1} \mathrm{H}-\mathrm{COSY}, \mathrm{HMQC}, \mathrm{HMBC}\right.$, and ROESY) NMR spectroscopy. Compounds 33, 34, and 37 showed cytotoxicity against astrocytoma and glioma cells (CCF-STTG1, CHG-5, SHG-44 and U251) with IC 50 values ranging from 9.2 to $17.4 \mu \mathrm{M}$. Adriamycin was used as positive control and showed cytotoxicity with an $\mathrm{IC}_{50}$ value ranging from 21.8 to $33.7 \mu \mathrm{M}$. These compounds exhibited a cytotoxic effect against breast cancer (MCF-7) and human skin cancer (SK-MEL-2) with $\mathrm{IC}_{50}$ values ranging from 28.1 to $35.5 \mu \mathrm{M}$. Adriamycin was used as positive control and exhibited a cytotoxic effect with $\mathrm{IC}_{50}$ values ranging from 14.1 to $37.6 \mu \mathrm{M}$ [39]. Alkaloids 35 and 38 displayed no cytotoxic activities or selective inhibition of COX-2 comparable to those of 33,34 and 37 although they possess the same monoterpene indole skeleton. The observations indicated that a $\mathrm{N}_{4}$-oxide functionality was essential for cytotoxic and anti-inflammatory properties, while a $N_{1}$-oxide maybe weaken the cytotoxic activities for this type of alkaloids. The observations indicated that the presence of oxide in $N_{4}$ was essential for cytotoxic and anti-inflammatory activities, while the presence of the oxide on $N_{1}$-oxide led to decreasing the cytotoxicity.
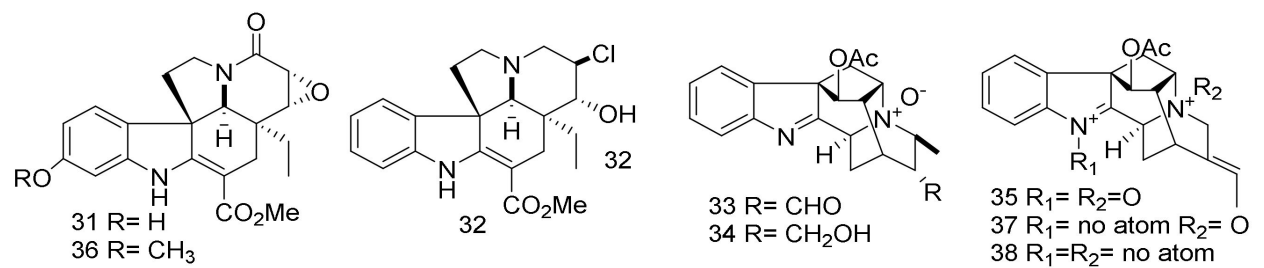

Figure 7. Compounds 31-38.

Alsmaphorazines (C) (39), (D), (40), and (E) (41) (Figure 8) were elucidated from $A$. pneumatophore [40]. The planar structure of 39 was elucidated by 2D NMR and MS. This alkaloid possesses a novel ring skeleton containing an octahydropyrrolo[2,3-b]pyrrole unit. The absolute configuration of (39) was determined by the modified Mosher's method and also confirmed by measuring the $C D$ spectrum, which fully agreed with the $C D$ calculations. Compounds 39-41 showed no cytotoxicity and also weak anti-melanogenesis activity against HL-60 and B16F10 cells with $\mathrm{IC}_{50}$ values $>100 \mu \mathrm{M}$ [40].

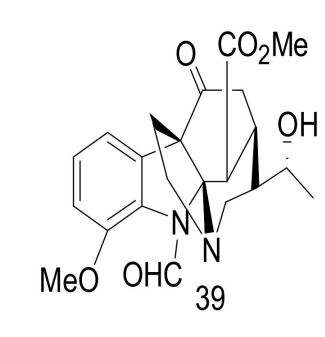

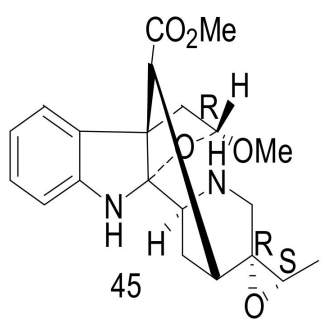

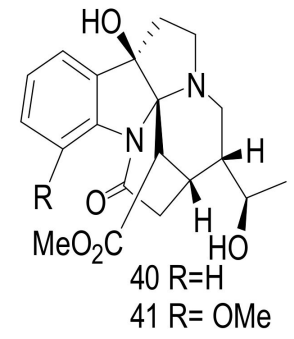

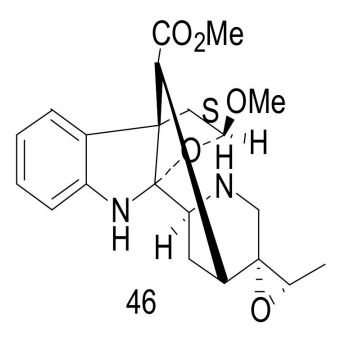

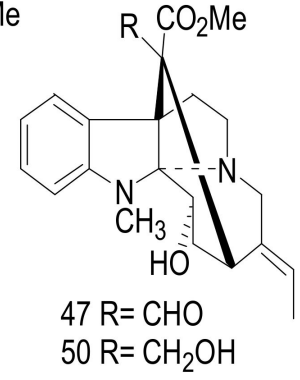

Figure 8. Compounds 39-51.

New scholarisins I-VII (42-48), and (3R,5S,7R,15R,16R,19E)-scholarisine F (49) [41], along with three known indoles: 3-epi-dihydrocorymine (50), and (E)-16-formyl-5 $\alpha$-meth oxystrictamine (51) were identified from the leaves of Alstonia rupestris (Figure 8) [42]. 
Compounds 42, 47, and 51 showed significant cytotoxicity against cancer cells, A-549, BGC-823, HepG2, HL-60, MCF-7, SMMC-7721, and SW480 with IC 50 values $<30 \mu \mathrm{M}$. These compounds exhibited selective inhibition effect of $\mathrm{COX}-2$ with $\mathrm{IC}_{50}$ values ranging between 92.0 and $96.4 \mu \mathrm{M}$, while compounds $\mathbf{4 3}, \mathbf{4 4}$, and $\mathbf{4 8}-\mathbf{5 0}$ displayed a weak cytotoxicity towards the tested tumor cells with $\mathrm{IC}_{50}$ values $>40 \mu \mathrm{M}$. Furthermore, alkaloids 45 and 46 showed a weak cytotoxicity with $\mathrm{IC}_{50}$ values $>80 \mu \mathrm{M}$. Doxorubicin was used as a positive control and showed with $\mathrm{IC}_{50}$ value $<35 \mu \mathrm{M}$. These activities of 45 and 46 , indicated that the bond connection between C-5 and N-4 was essential for the cytotoxicity [41]. Compounds 42, 43, 44 and 49 showed antifungal activity against Gibberella pulicaris (KZN 4207) and Colletotrichum nicotianae (SACC-1922) with MIC values of 0.64 and $0.69 \mathrm{mM} ; 1.37$ and $1.44 \mathrm{mM} ; 1.80$ and $1.91 \mathrm{mM}$ and 1.55 and $1.71 \mathrm{mM}$, respectively. Nystatin was implemented as a positive control and showed MIC values of 0.007 and $0.006 \mathrm{mM}$. These bioactivities may be due to the presence of a formyl group at $\mathrm{C}-16$ in the alkaloids subclasses picrinine in $\mathbf{4 2}$, vincorine in $\mathbf{4 7}$, and akuammiline in 51 , respectively and also may play a role in anti-inflammatory activity [41].

Alstolactines A (52), B (53), and C (54) (Figure 9) were isolated from the leaves of chines $A$. scholaris [43]. The structures were identified by extensive spectroscopic data analyses and X-ray diffraction analyses. The absolute stereochemistry was deduced from crystal X-ray diffraction. These compounds are biosynthetically originated from picrinine, which is the main metabolite in A. scholaris. Compounds 52-54 exhibited no effects against four bacterial strains: Klebsiella pneumonia, Escherichia coli, Pseudomonas aeruginosa and Staphylococcus aureus [43].

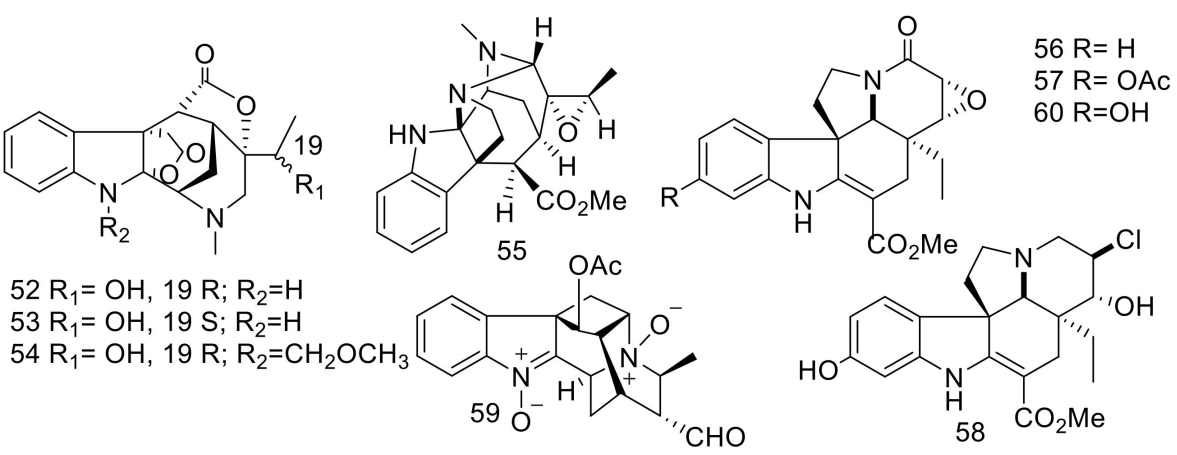

Figure 9. Compounds 52-60.

Moreover, Alistonitrine A (55) (Figure 9) had an unprecedented caged carbon skeleton with a unique $6 / 5 / 6 / 5 / 5 / 6$ ring system and also contained three nitrogen atoms. It was isolated from the same species [12]. Its structure and absolute configuration were established by extensive spectroscopic analyses and electron circular dichroism calculations. Compound 55 exhibited no activity as an anti-inflammatory in both NF- $\mathrm{KB}$ and HIF- $\alpha$ models [12].

The MIAs, 6,7-epoxy-8-oxo-vincadifformine (56), 11-acetyl-6,7-epoxy-8-oxo-vincadiff ormine (57), 11-hydroxy-14-chloro-15-hydroxyvincadifformine (58) and perakine $N_{1}, N_{4}$ dioxide (59) were identified from the aerial parts of A. rupestris. Additionally, 11-hydroxy6,7-epoxy-8-oxovincadifformine (60) and $\mathbf{3 5}$ were isolated from the same species [44].

Compounds 56, 57 and 60 exhibited potent cytotoxic effects against head and neck squamous cancer (SCL-1, Detroit-562, UMSCC-1, CAL-27, TCA-83, HepG2 and SCC-PKU) cells, with $\mathrm{IC}_{50}$ values $<20 \mu \mathrm{M}$. Doxorubicin was implemented as a positive control and showed cytotoxicity, with $\mathrm{IC}_{50}$ values $\leq 35.4 \mu \mathrm{M}$. Compound 56 exhibited potent effect, with $\mathrm{IC}_{50}$ values $\leq 13.7 \mu \mathrm{M}$. This may be due to the absence of any substitution at the phenolic ring. This can be explained by the fact that the attachment of electron-donating groups (OH and $\mathrm{OAc}$ ) led to a reduction in the cytotoxicity [44]. Compounds 56, 57, and 60 displayed significant antifungal activities against Alternaria alternata and Phytophthora capsici, with MIC values $=0.66 \& 0.99 \mathrm{mM}, 0.87 \& 1.10 \mathrm{mM}$ and $1.53 \& 1.64 \mathrm{mM}$, respectively. 
Nystatin was implemented as positive control and showed effect with MIC values 0.007 and $0.061 \mathrm{mM}$. Compounds 56, 57, and 60 displayed moderate activity against Staphylococcus aureus, with MIC values of 15.72, 16.33 and $14.91 \mathrm{mM}$. Meanwhile, compounds 59 and 35 exhibited potent effects against Staphylococcus aureus, with MIC values of 0.49 and $0.83 \mathrm{mM}$. Rifampicin was used as a positive control and showed an effect at MIC valued $=0.003 \mathrm{mM}$ for bacteria. Additionally, compound 59 showed higher antibacterial effects toward S. aureus than compound 35. The present of a formyl group at the C-20 position might increase the activities for ajmaline indole alkaloids [44].

The bioassay-guided fractionation of the stem bark of Vietnamese Alstonia angustifolia using the HT-29 human colon cancer cells, led to the reporting of six MIAs, $N_{(4)}$-methyltalpinine (61) [45], $N_{(4)}$-meth-yl- $N_{(4)}$,21-secotalpinine (62) [46], alstonerinal (63) [47], alstonerine (64) [48], macrocarpine B (65) [46], affinisine (66) [49], from the stem bark of $A$. angustifolia. Additionally, villalstonine (67), villalstonine $N_{(4)}$-oxide (68) [50], villalstonidine D (69) and villalstonidine E (70) [51] (Figure 10) were identified from the same plant.

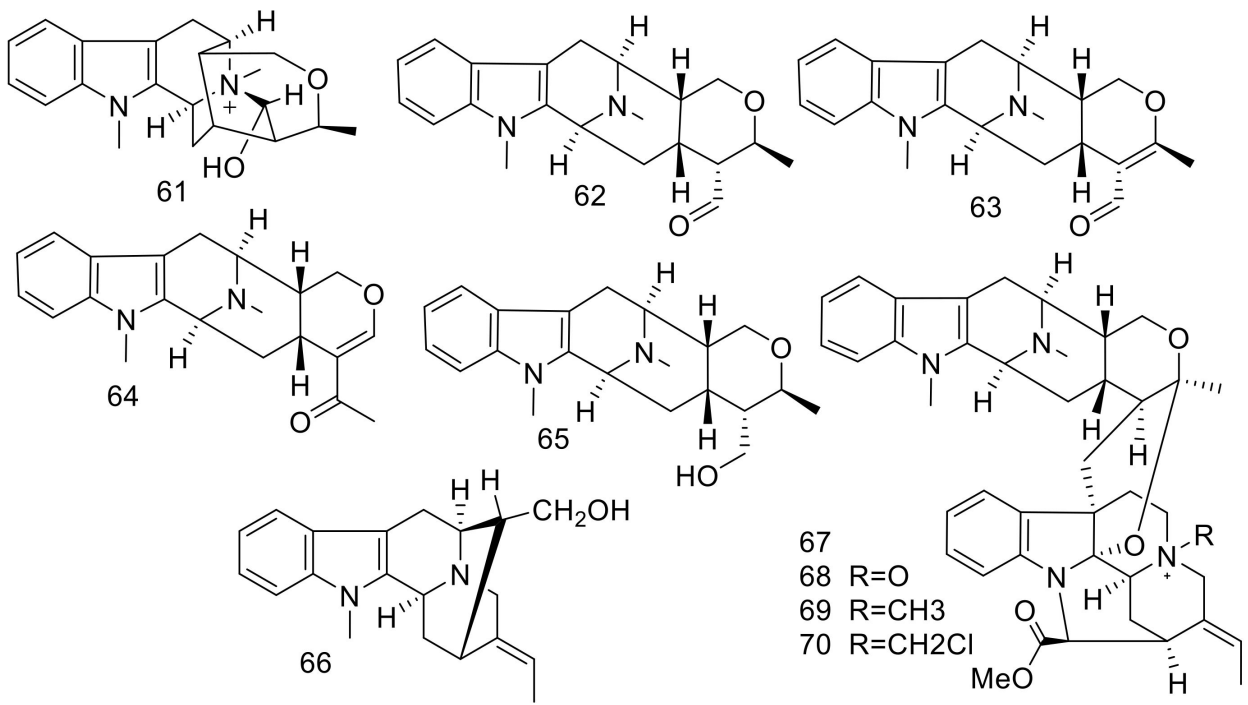

Figure 10. Compounds 61-70.

Compounds 61 and 66 are sarpagine-type and compounds 62-65 are macrolinederived alkaloids whereas macroline-pleiocarpamine bisindole alkaloids are present in compounds 67-70.

Compound 61 showed significant inhibitory activity toward NF-kB (p65), with an $\mathrm{ED}_{50}$ value $=1.2 \mu \mathrm{M}$. Rocaglamide was employed as a positive control, with $\mathrm{ED}_{50}$ value $=0.9 \mu \mathrm{M}$. Compounds 61-64, 66 and 68-70 showed anti-leishmanial activity toward the promastigotes of Leishmania Mexicana, with $\mathrm{IC}_{50}$ values $<183.5 \mu \mathrm{M}$. Compound 62 exhibited a potent effect, with $\mathrm{IC}_{50}$ value $=57.8 \mu \mathrm{M}$. Amphotericin B was employed as a positive control and exhibited potent effect against L. mexicana promastigote, with an $\mathrm{IC}_{50}$ value $=0.09 \mu \mathrm{M}$. The dimeric compounds 68-70, which contain quaternary ammonium cation at $N(4)$, exhibited potent effect than compound 67. Additionally, compound 67 has not function group at $N_{(4)}$ [45]. Also, the presence of formyl and acetyl groups in 62-64. These moieties may enhance the effects of compounds belonging to macroline indole alkaloids compared with 65.

Normavacurine-21-one (71), 5-hydroxy-19, 20-E-alschomine (72), and 5-hydroxy-19, 20-Z-alschomine (73) (Figure 11), were isolated from the leaves of Alstonia scholaris cultivated in Kunming, China [52]. Compound 71 exhibited a significant antimicrobial effect against Enterococcus faecalis ATCC 10541, with an MIC $=0.78 \mu \mathrm{g} / \mathrm{mL}$, whereas compound 73 showed a significant effect against Pseudomonas aeruginosa ATCC 27853, with an $\mathrm{MIC}$ value $=0.781 \mu \mathrm{g} / \mathrm{mL}$. Cefotaxime was used as a positive control, with an MIC = $0.19 \mu \mathrm{g} / \mathrm{mL}$ [52]. Alstoniascholarines A-Q (74-90), were identified from the leaves of $A$. 
scholaris collected from Yunnan [53,54]. Compounds 79 and 83 showed a potent antibacterial activity against Pseudomonas aeruginosa ATCC 27853, with MIC value $=3.13 \mathrm{mg} / \mathrm{mL}$. Gentamycin was applied as a Positive control and showed an inhibitory effect, with an MIC value $=0.78 \mathrm{mg} / \mathrm{mL}$. Additionally, compounds 77, 80, and 83 exhibited moderate antifungal activities toward Epidermophyton floccosum CBS 566.94, with MIC value s=31.25 mg $/ \mathrm{mL}$. Griseofulvin was applied as a positive control and showed an inhibitory effect, with an MIC value $=7.81 \mathrm{mg} / \mathrm{mL}$ [53]. Compounds $\mathbf{8 5}-\mathbf{9 0}$ showed no cytotoxicity against five tumor cell: MCF-7, A-549, HL-60, SW-480, and SMMC-7721[54].
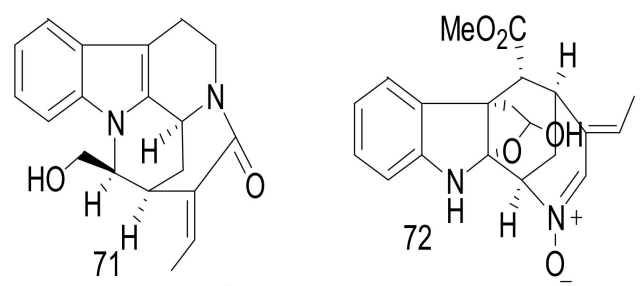

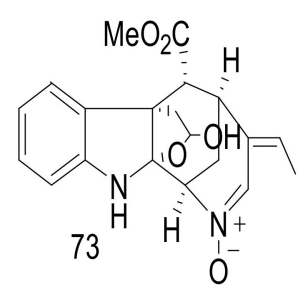

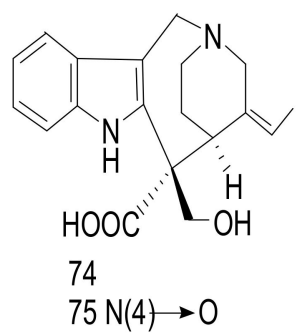

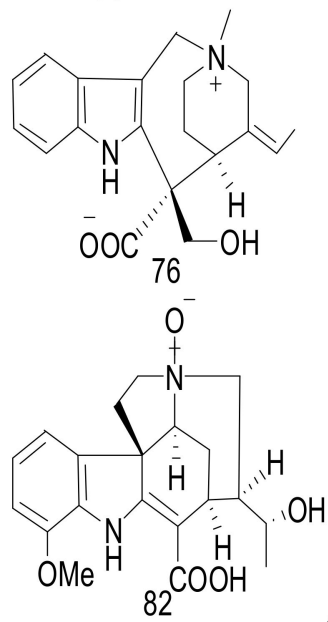<smiles>C[N+]1(C)CC[C@H]2C(=COCc3c2[nH]c2ccccc32)CO1</smiles>

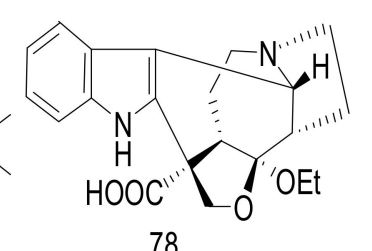<smiles>[R]c1cccc2c1NC1=C(C(=O)O)[C@@H](C(C)O)[C@@H]3C[C@H]1CN3CC2</smiles>

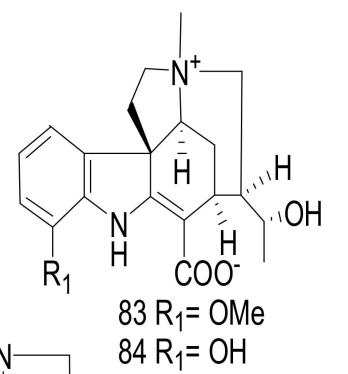

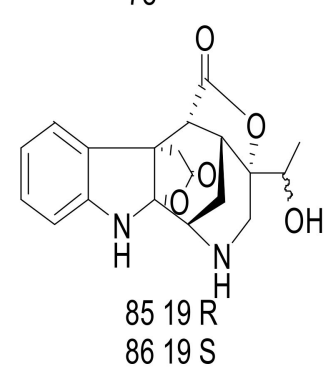

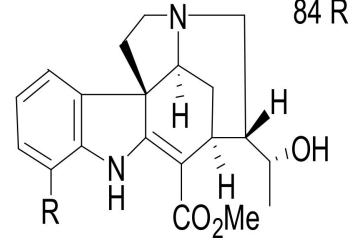

\section{$88 \mathrm{R}=\mathrm{OH}$} $89 \mathrm{R}=\mathrm{OH} \mathrm{N}(4) \rightarrow 0$ $90 \mathrm{R}=\mathrm{OMe} \mathrm{N}(4) \rightarrow 0$ $8619 \mathrm{~S}$

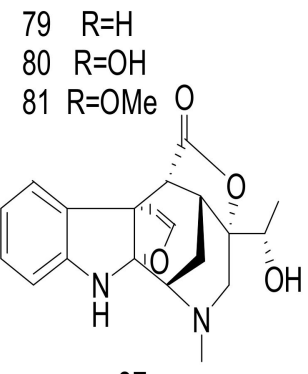

87

Figure 11. Compounds 71-90.

Scholarisines H-O (91-97) (Figure 12) were isolated from the leaves of the Chinese A. scholaris [55]. The chemical structures were elucidated on the basis of comprehensive spectroscopic data and X-ray diffraction. Compounds 91-97 showed weak antibacterial activities against five strains: Pseudomonas aeruginosa ATCC 27853, Staphylococcus aureus ATCC 25922, Escherichia coli ATCC 11775, Providencia smartii ATCC 29916, and Enterococcus faecalis ATCC 10541), with MIC values $=100 \mu \mathrm{g} / \mathrm{mL}$. Gentamycin was used as a positive control, with an MIC value $<2.00 \mu \mathrm{g} / \mathrm{mL}$ [55].
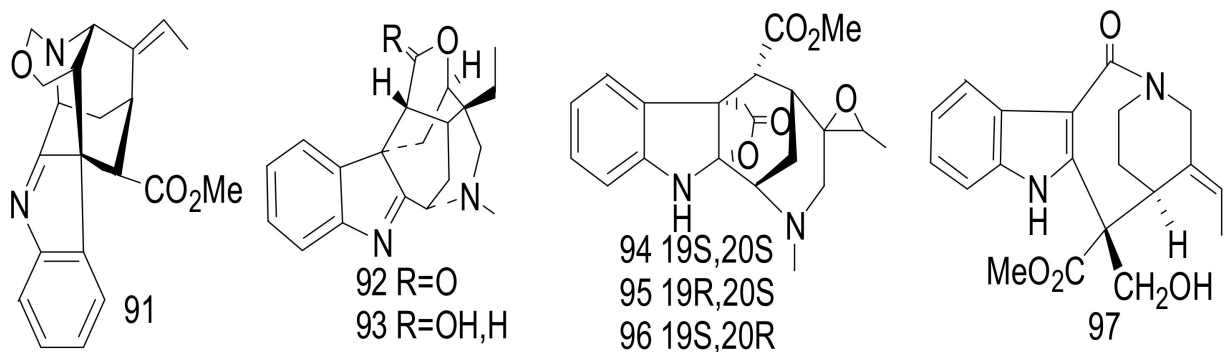

Figure 12. Compounds 91-97. 
A further study on the leaves and twigs of $A$. scholaris [56] led to identification of melosline A (98), B (99) and 1-[2-[2-(carboxymethyl) indole-3-yl] ethyl]-3-ethylpyridinium hydroxide inner salt (100) (Figure 13) [57]. Melosline A (98) was an unprecedented indole alkaloid, with a $6 / 5 / 6 / 6$ tetracyclic ring skeleton. The structures were established by spectroscopic analyses. The absolute configuration of 98 was confirmed by the comparison of experimental data with the calculated electronic circular dichroism (ECD). Compound 98 showed a moderate cytotoxic activity against breast cancer (MCF-7), with an $\mathrm{IC}_{50}$ value $=39.78 \mu \mathrm{M}$. Cisplation was employed as a positive control [56].

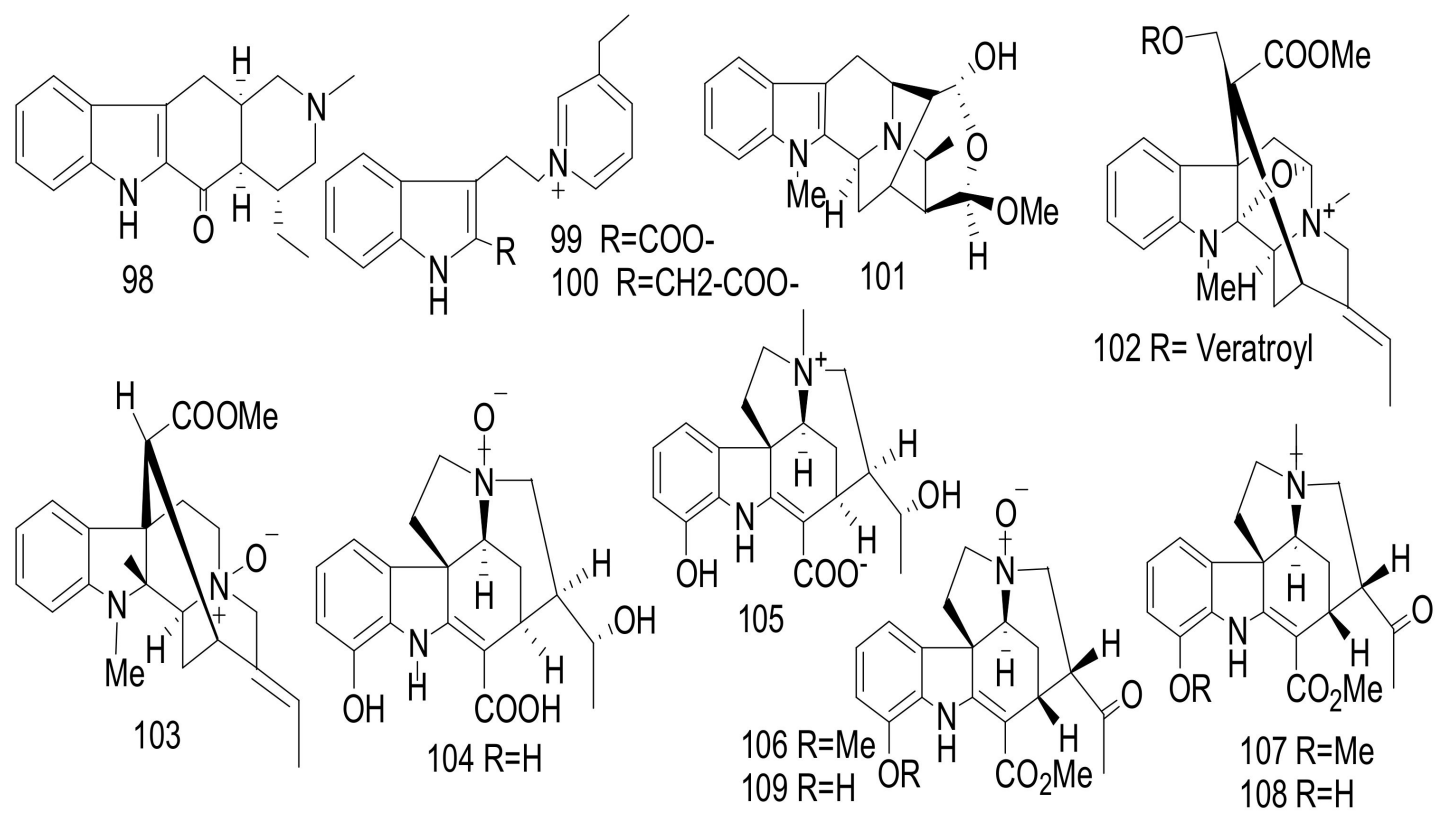

Figure 13. Compounds 98-109.

Alstiyunnanenines A-E (101-105) (Figure 13) and alstoniascholarine I (82) (Figure 11), were isolated from A. yunnanensis [54,58]. Compounds 104, 105, and 82 displayed potent cytotoxicity against human gastric carcinoma (BGC-823 cells), human hepatocellular, (HepG2 cells), human myeloid leukemia (HL-60), human breast cancer (MCF-7), and osteosarcoma (SOSP-9607, MG-63, Saos-2, M663), with $\mathrm{IC}_{50}$ values ranging between 3.2 and $5.8 \mu \mathrm{M}$. Adriamycin was used as a positive control and exhibited cytotoxicity, with an $\mathrm{IC}_{50}$ value $<0.04 \mu \mathrm{M}$ [58]. Three monoterpenoid indoles, alstomairines A-C (106-108) [59], together with alpneumine A (109) [60] were identified from the leaves of the chines $A$. mairei. Compounds 107 and 108 showed potent cytotoxic effects against osteosarcoma cells (U2-OS, Mg-63, Saos-2, and SOSP-9607) with IC 50 values ranging from 9.2 to $13.0 \mu \mathrm{M}$, whereas compounds 106 and 109 had $\mathrm{IC}_{50}$ values $<15.0 \mu \mathrm{M}$. The presence of the methyl group on $\mathrm{N}_{-}$indicate increasing the cytotoxicity in that scholaricine-type (Figure 4) than the presence of $N_{(4)}$-oxide moiety. Doxorubicin was used as Positive control and showed cytotoxicity, with an $\mathrm{IC}_{50}$ value $<0.03 \mu \mathrm{M}$ [59].

Alstrostine G-K (110-114) (Figure 14), were identified from the Chinese A. rostrata [61]. Compounds 110-114 showed no cytotoxicity against HeLa, SGC-7901 gastric cancer, and A-549 lung cancer at $20 \mu \mathrm{M}$ [61]. 


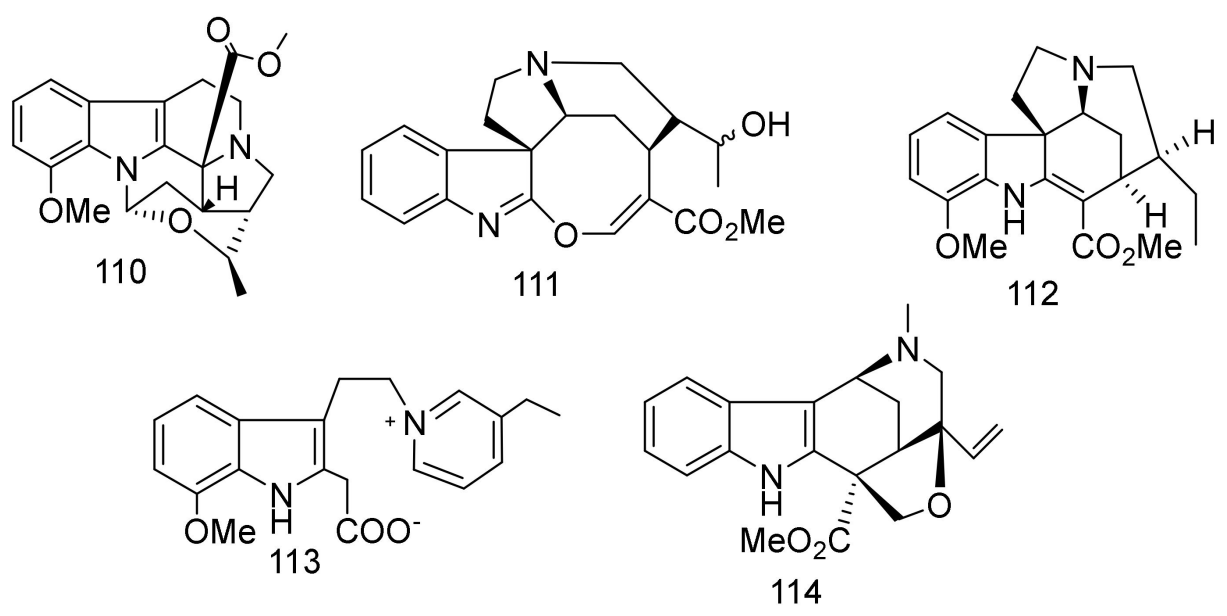

Figure 14. Compounds 110-114.

Six nareline-type indoles including three cage-like skeletons, scholarisines T-V (115117) [62] (Figure 15), and three previously identified analogues scholarisine $W$ (118), scholarisine A (119), and scholarisine I (92) [55], were isolated from the leaves of the Chinese A. scholaris [56]. Compounds 115-117 displayed anti-bacterial effects against Escherichia coli ATCC 8739, with an MIC value $=0.78 \mu \mathrm{g} / \mathrm{mL}$. Additionally, compound (116) inhibited the growth of Bacillus subtilis ATCC 6633 bacterium with an MIC value $=3.12 \mu \mathrm{g} / \mathrm{mL}$ and was referenced with cefotaxime as a positive control. The absence of the ethyl group at C-20 position indicated an increase in the anti-bacterial activities as in 116, compared with compounds (115 and 117) [63]. Cefotaxime was used as a positive control and exhibited an inhibitory effect, with an MIC value of $0.39 \mu \mathrm{g} / \mathrm{mL}$. There were scholarisines P-S (120-123), (16R)-E-isositsnikine (124) [64], nareline (125) [65], 5-methoxystrictamine (126), leuconolam (127), epileuconolam (128) [66], and $N^{\mathrm{b}}$-demethylalstogustine (129) [67]. Also, 19-epischolaricine (130), scholaricine (131), vallesamine (132) [68], akuammidine (133) [69], 17-nor-excelsinidine (134) [70], strictosamide (135) [71,72] and compounds 19 and 21, were isolated from the same species. Compounds 123, 19, 21, 130 and 133 exhibited significant NF- $\mathrm{kB}$ inhibitory activity with $\mathrm{IC}_{50}<25 \mu \mathrm{M}$. Furthermore, compounds 19, 126 and 130 inhibited TNF $\alpha$-induced NF- $\mathrm{KB}$ activation in the same dose. Three nareline-type MIAs, compounds (120, 123 and 125) were identified from A. scholaris [73].

Two ajmaline type MIAs, vincamaginine A (136), and vincamaginine B (137); four macroline oxindole- alstonisinines A (138) and B (139), alstonisinine C (140), and alstonoxine $\mathrm{F}$ (141); four bisindole compounds of macroline-akuammiline type; angustilongine A-D (142-145) (Figure 16) were reported from Malaysian Alstonia penangiana [73]. The structures of these alkaloids were determined by the interpretation of spectroscopic data and compounds 141-142, were confirmed by X-ray diffraction analysis. Compounds 142 and $\mathbf{1 4 3}$ showed growth inhibitory activity against human prostate carcinoma (LNCaP and PC-3), human breast adenocarcinoma (MDA-MB-231 and MCF7), human colorectal carcinoma (HCT 116 and HT-29) and human lung carcinoma (A549). Furthermore, the potent effects of 142 and 143 against HT-29 cells were evaluated, with $\mathrm{IC}_{50}$ values $=0.7 \pm 0.1 \mu \mathrm{M}$ and $0.3 \pm 0.0 \mu \mathrm{M}$, respectively (Cisplatin, $\mathrm{IC}_{50}>10 \mu \mathrm{M}$ ). Compound 143 exhibited an effect against vincristine-resistant $\mathrm{KB}$ cells, with an $\mathrm{IC}_{50}$ value of $0.7 \pm 0.3 \mu \mathrm{M}$ (Vincristine $0.3 \pm 0.1 \mu \mathrm{M})[73]$. 


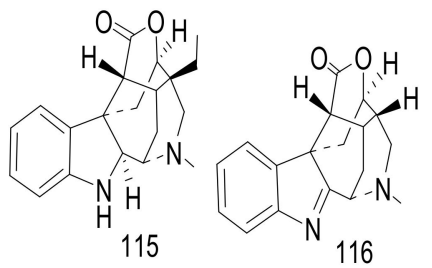

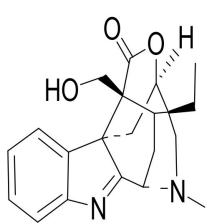

120

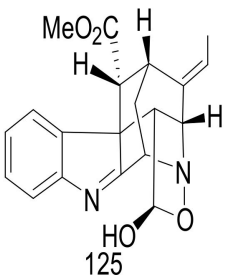

125

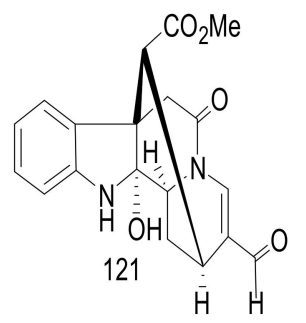<smiles>[R20]CC(C(=O)OC)[C@H]1C[C@@H](/C=C/C)CN2CC[C@@H]3C(=Nc4ccccc43)[C@H]12</smiles>

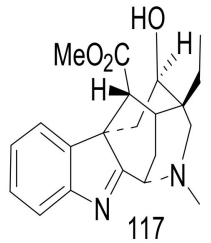

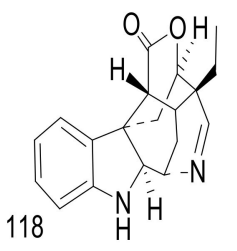

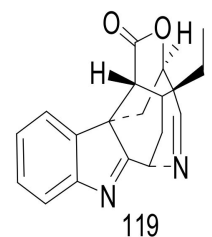

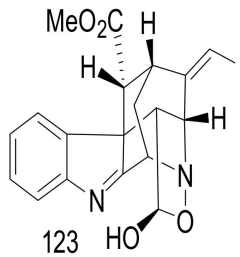

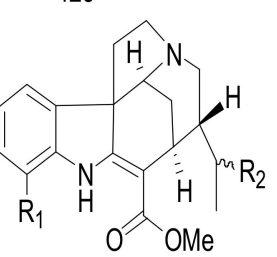

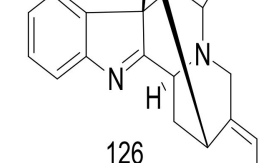<smiles>[R]C12CCCN3C(=O)C=C(Oc4ccccc4N1)C3(CC)CC2</smiles>

$129 \mathrm{R}_{1}=\mathrm{H} \quad \mathrm{R}_{2}=\alpha-\mathrm{OH}$ $130 \mathrm{R}_{1}=\mathrm{OHR} \mathrm{R}_{2}=\alpha-\mathrm{OH}$ $127 \mathrm{R}=\alpha_{-} \mathrm{OH}$

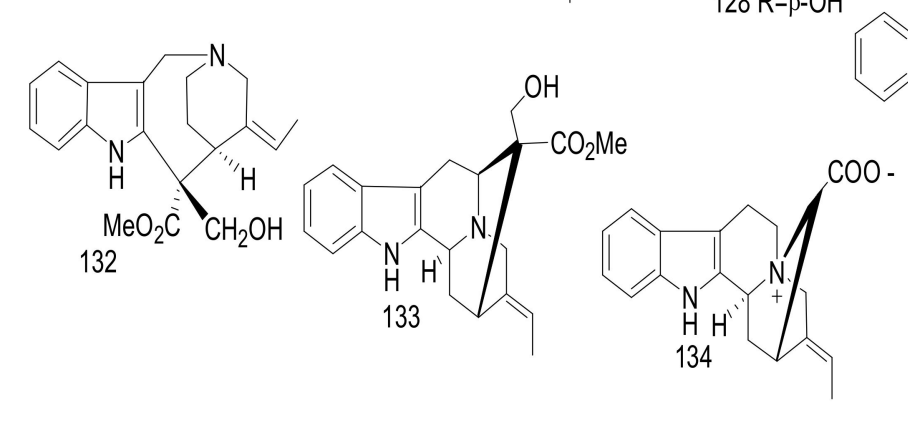
$131 \mathrm{R}_{1}=\mathrm{OH} \mathrm{R}=\beta-\mathrm{OH}$<smiles></smiles>

Figure 15. Compounds 115-135.

Winphyllines A (146), B (147) [74], $N_{b}$-demethylechitamine (148) [75], 17-O-acetylnore chitamine (149) (Figure 17) [76], 12-methoxyechitamidine (150) [67], and $N_{(4)}$-demethylasto gustine (151) [77] were isolated from the collected twigs of the Chinese A. rostrata. Compounds 146-151 exhibited cytotoxicity against cancer cells (HL-60, SMMC-7721, A-549, MCF-7, and SW480), with $\mathrm{IC}_{50}$ values $=40 \mu \mathrm{M}$ [74]. A vincorine-type, 17-formyl-10demethoxyvincorine $N_{(4)}$-oxide (152), an ajmaline-type 10-methoxyalstiphyllanine $\mathrm{H}(\mathbf{1 5 3})$, and 10-demethoxyvincorine $N_{(4)}$-oxide (154) were obtained from the leaves of $A$. scholaris [78]. The phytochemical investigation of $A$. scholaris led to the publication of alstoscholactine (155) and alstolaxepine (156) [79]. A further investigation on the leaves of Malaysian A. scholaris led to the reporting of alstobrogaline (157) [80]. Compounds 155 and 156 exhibited no cytotoxic effects, whereas 156 induced marked vasorelaxation in reported rat aortic rings precontracted with phenylephrine, with $\mathrm{EC}_{50}=6.58 \pm 3.66 \mu \mathrm{M}$ and Emax $=93.9 \pm 4.3 \%$ (cf. verapamil, $\mathrm{EC}_{50}=0.55 \pm 0.19 \mu \mathrm{M}$ and Emax $=106.4 \pm 3.4 \%$ ) [74] Compound 157 showed weak cytotoxic activity against breast cancer cells MDA-MB-231 and MCF7, with $\mathrm{IC}_{50}$ values $=25.3$ and $24.1 \mu \mathrm{M}$, respectively [80]. 


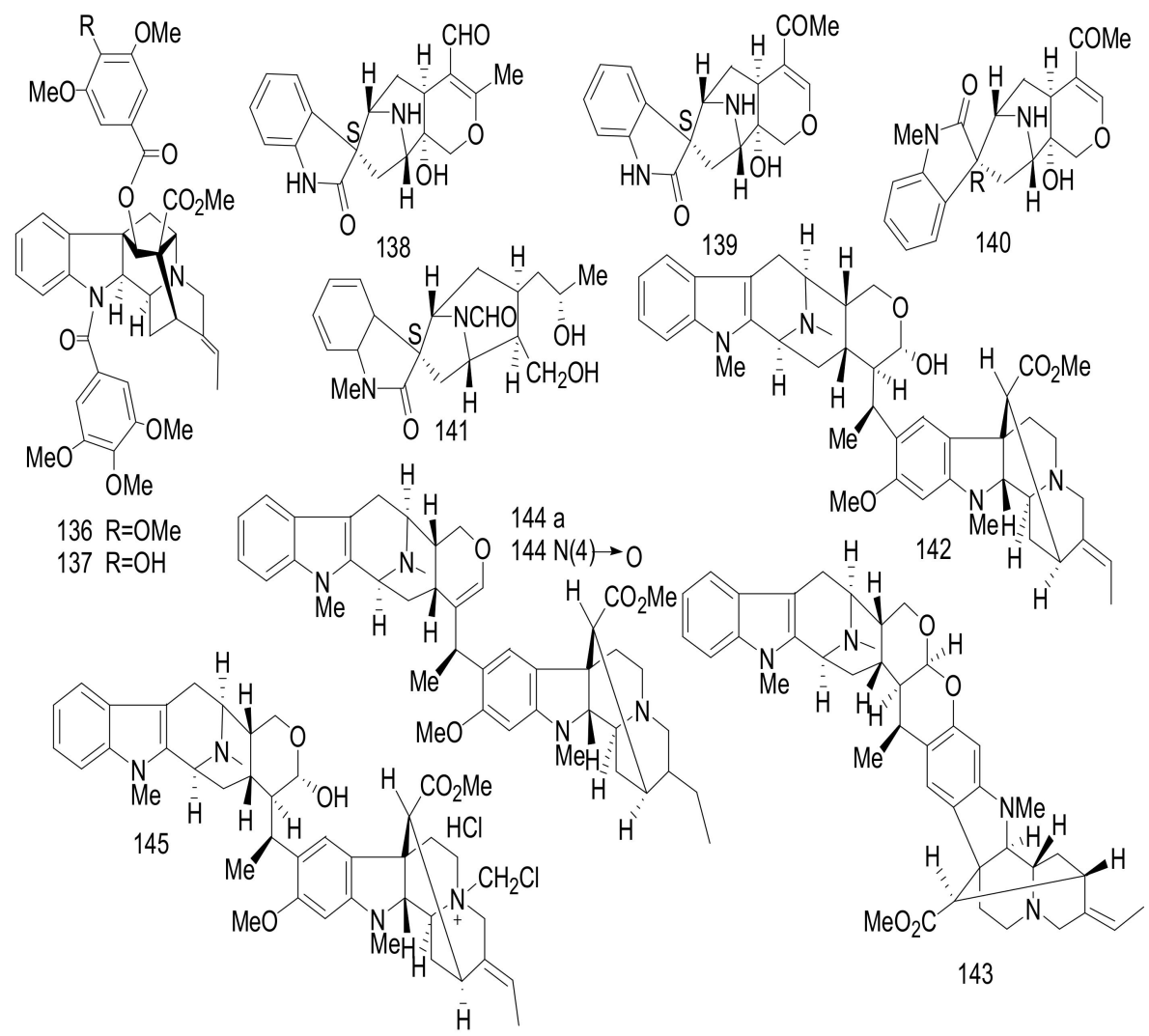

Figure 16. Compounds 136-145.

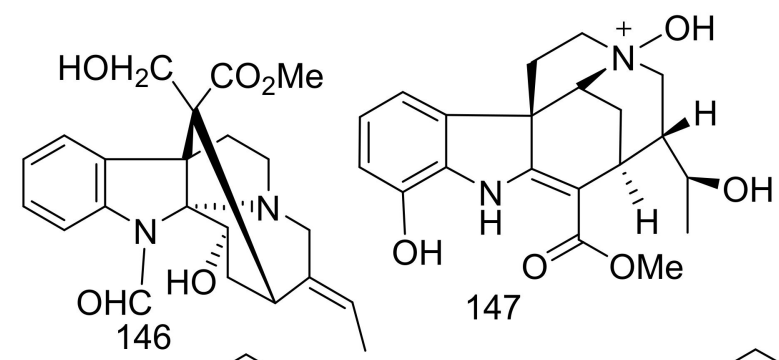<smiles>COC(=O)C1=C2Nc3c(OC)cccc3[C@]23CCN2C[C@H](C(C)O)[C@H]1CC23</smiles><smiles>COC(=O)C1=C2Nc3ccccc3[C@]23CCN2C[C@H]([C@H](C)O)[C@H]1C[C@H]23</smiles>

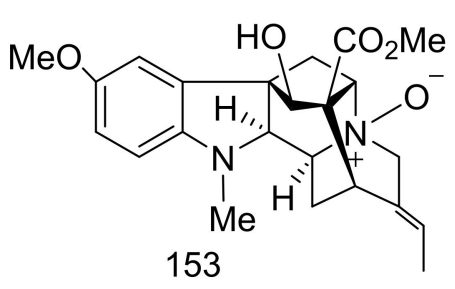

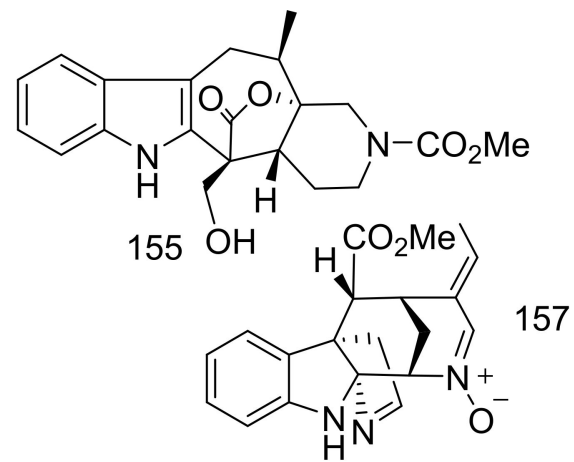

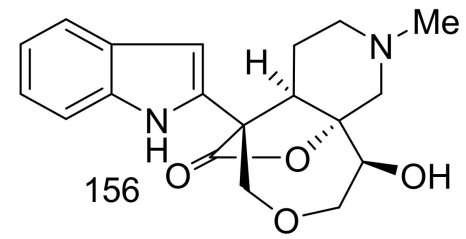

Figure 17. Compounds 146-157. 
The scaffold of the reported monoterpene indole compounds from A. scholaris is affected by the geographical environment. Indian, Pakistanian and Thai. $A$. scholaris are rich with picrinine-type indole compounds, whereas, those identified from Indonesia and the Philippine, are rich in angustilobine-type [81]. Genus Alstonia was addressed as a source of angustilongines A (142) and B (143). These compounds showed more potent anticancer activities than those recognized from $A$. penangiana, although all of them belong to macroline- and akuammiline-type bisindole alkaloids.

A review entitled "Alstonia scholaris and Alstonia macrophylla: A comparative review on traditional uses, phytochemistry and pharmacology" was published in 2014 and mentioned the compounds obtained from A. scholaris from 1976 to 2009, and from A. macrophylla from 1987 to 2013 [82]. A review published in 2018 entitled "The alstoscholarisine compounds: isolation, structure determination, biogenesis, biological evaluation and synthesis" studied the alstoscholarisine compounds obtained from $A$. scholaris [83]. Furthermore, a review published in 2016 called "An overview phytochemistry and chromatographic analysis of Alstonia scholaris used as a traditional medicine" discussed A. scholaris compounds which were reported between 1965 and 2009 [84].

The identified metabolites from Alstonia were categorized under two main classes: corynanthe and aspidosprma. Corynanthe contains eight subclasses: ajmaline-type (18, and 33-35), picrinine-type (19-21 and 42-44), akummiline-type (22), vincorine-type (47, 50 and 148-149), sarpagine-type (61, 66 and 101), macroline-type (62-65), scholaricinetype (104-109) and macroline oxindole-type (138-141). Meanwhile,, aspidosprmia contains six subclasses: aspidosprma-type (31 and 32) vincamine-type (82-84 and 88-90), aspidofractinine-type (1 and $\mathbf{4}$ ), bisindole alkaloids macroline-pleiocarpamine-type (67-70), and macroline- akuammiline-type (142-145). Ajmaline derivatives with formyl group and/or a quaternary ammonium cation $N(4)$ showed an interesting bioactivity.

\section{Kopsia}

Kopsia (Family Apocynaceae) contained 30 species with a distribution in China, India, Southeast Asia, and Australia. Sixteen species were grown in Malaysia [85], and five species were grown in Thailand [86]. These plants are considered as rich sources of indolecontaining compounds. Traditionally, some of the species have been used for the treatment of tonsillitis, dropsy and rheumatoid arthritis. Several species have been reported to have antitumor, antimanic, antitussive and antileishmanial effects [87-89]. A review published in 2017 was interested in reporting indole alkaloids from genus kopsia plants regarding reversing multidrug resistance in vincristine-resistant $\mathrm{KB}$ cells for example, kopsirensine $\mathrm{B}$, arboloscine A [90], grandilodines A and C, and lapidilectine B [91,92].

Kopsiyunnanines G (158) and kopsiyunnanines H (159) (Figure 18) with an aspidospermacontaining skeleton were isolated from the aerial part of the Chinese Kopsia arborea [93]. Kopsihainins A (160), B (161), and C (162) were isolated as new compounds from K. hainanensis [89], along with the known compounds, kopsinine (163) [87] and methyl demethoxycarbonylchanofruticosinate (164) [94] were isolated from the stems of Chinese K. hainanensis. Compounds 163 and 164 showed significant antitussive activity, these compounds are within the aspidofractinine-type and methyl chanofruticosinate-type indoles, respectively. Compounds 163 and 164 inhibited coughing by 88\% and 76\%, respectively [83]. Compound 163 was more active, with an ID 50 value $=0.11 \mathrm{mmoL} / \mathrm{kg}$, whereas compound 164 exhibited an effect, with an $\mathrm{ID}_{50}$ value $=0.45 \mathrm{mmol} / \mathrm{kg}$, (Codeine, $\mathrm{ID}_{50}=0.1 \mathrm{mmol} / \mathrm{kg}$ ) [90]. The link from C-2 to C-20 in compound 163 and the attachment of the methoxy carbonyl group at C-16 position promote the antitussive activity. 

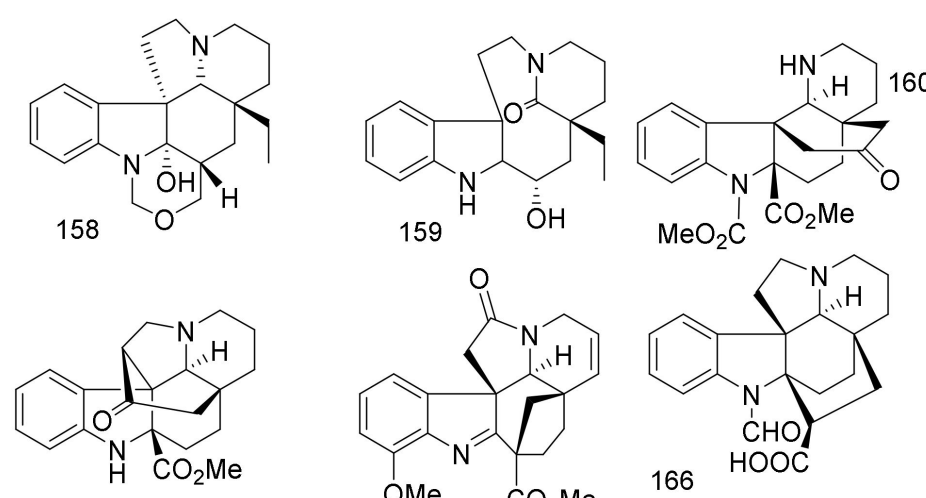

164

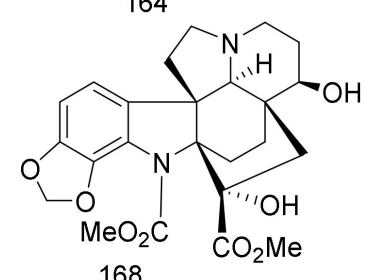<smiles>COC(=O)C1=Nc2c(OC)cccc2[C@@]23CC(=O)N4CC=C[C@]5(CCCC12[C@@H]45)C3</smiles>

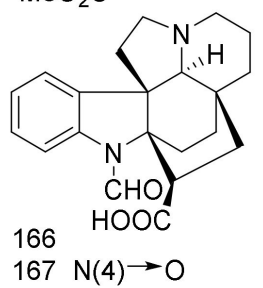<smiles>[R2]C1CCC23CCCCC2(CC1)c1ccccc1N3</smiles>

$161 \mathrm{R}_{1}=\Delta 14,15, \mathrm{R}_{2}=\mathrm{COOH}$ $162 \mathrm{R}_{1}=\beta-\mathrm{O}-, \mathrm{R}_{2}=\mathrm{COOH}$ $163 \mathrm{R}_{1}=$ nil, $\mathrm{R}_{2}=\mathrm{CO}_{2} \mathrm{Me}$

165<smiles>COC(=O)C1=C2Nc3ccccc3[C@]23CC[N+]2(C)CC[C@@H](O)[C@@]13C2(C)C</smiles>

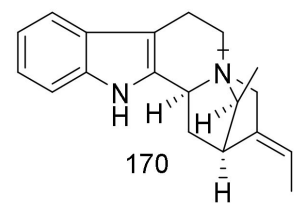

Figure 18. Compounds 158-170.

Four alkaloids of aspidofractinine-type, singaporentine A (165), $N_{(1)}$-formylkopsininic acid (166), $N_{(1)}$-formylkopsininic acid- $N_{(4)}$-oxide (167), and 15-hydroxykopsamine (168), along with an aspidospermatan-type, $14 \alpha$-hydroxy- $N_{(4)}$-methylcondylocarpine (169), and singaporentinidine (170) (Figure 18) were identified from the barks and leaves of Malaysian K. singapurensis [95].

From the leaves and stems of the Chinese medicinal plant $K$. hainanensis, four compounds, kopsininate (171), $N_{1}$-decarbomethoxy chanofruticosinic acid (172), methyl $N_{1}$ decarbomethoxy chanofruticosinate $N_{(4)}$-oxide (173) and methyl chanofruticosinate $N_{(4)^{-}}$ oxide (174) (Figure 19) were reported [96]. Compound $\mathbf{1 7 2}$ was the most effective against Erwinia carotowora bacterium, with an MIC of $7.8 \mathrm{mg} / \mathrm{mL}$. Furthermore, compound 172 showed antifungal activities against four plant pathogenic fungi: Penicillium italicum, Fusarium oxysporum $\mathrm{f}$. $\mathrm{sp}$. Niveum, Rhizoctonia solani and Fusarium oxysporum. Cubense had an $\mathrm{EC}_{50}=$ from 15.2 to $43.8 \mu \mathrm{g} / \mathrm{mL}$ dose values. Compound 172 showed a potent effect towards F. oxysporum f. sp. Cubense, with an $\mathrm{EC}_{50}=15.2 \mathrm{mg} / \mathrm{mL}$. A comparison of this result with the positive control Midlothian, with an $\mathrm{EC}_{50}=57.0 \mathrm{mg} / \mathrm{mL}$ showed compound 172 to be more active. The presence of carboxylic group attached to the C-2 position in $\mathbf{1 7 2}$ is important for antifungal activity, particularly, in methyl chanofruticosinate-type indoles [96].

Three aspidofractinie-type compounds, 5,6-secokopsinine (175), 5ß-hydroxykopsinine (176), 16-epi-kopsinilam (177) [97], two kopsine-type metabolites, 5-oxokopsinic acid (178), and $N_{\mathrm{a}}$-demethoxycarbonyl-12-methoxykopsine (179) [97], a strychnos-type, 14(S)hydroxy-19(R)- methoxytubotaiwine (180), and vincamine-type, and strychnos type 19oxo-(-)-eburnamonine (181), 19(S)-hydroxy- $\Delta^{14}$-vincamone (182) [97], along with ten known compounds, 163 [87], kopsinilam (183) [98], kopsinic acid (184), 12-methoxykopsine (185) [99], kopsanone 186), 19(R)- methoxytubotaiwine (187) [88], (-)-eburnamonine (188), 19-OH-(-)-eburnamonine (189), and $\Delta^{14}$-vincamone (190) [97] were yielded from the stem bark of the Thai Kopsia jasminiflora (Figure 19). Compounds 163, 183, and 184 belong to aspidofractinie-type, 185 and 186 belong to Kopsine-type, 187 belongs to strycno-type, 188-190 belonging to the vincamine- type MIAs. 


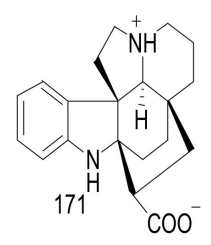

(n)

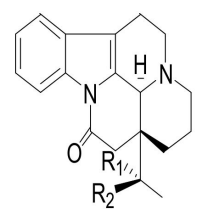

$181 R_{1}+R_{2}=0$

$188 \mathrm{R}_{1}=\mathrm{H}, \mathrm{R}_{2}=\mathrm{H}$

$189 \mathrm{R}_{1}=\mathrm{OH}, \mathrm{R}_{2}=\mathrm{H}$

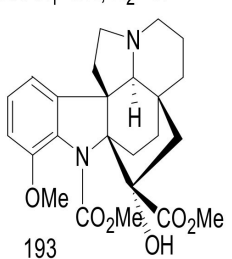

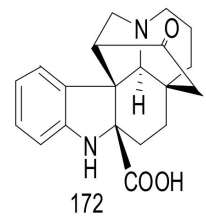
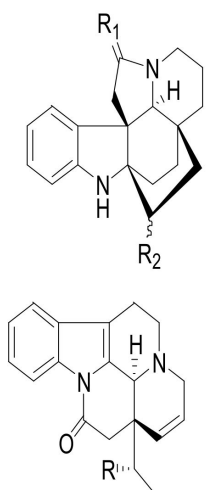

$182 \mathrm{R}=\mathrm{OH}$ $190 \mathrm{R}=\mathrm{H}$

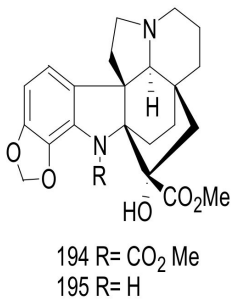

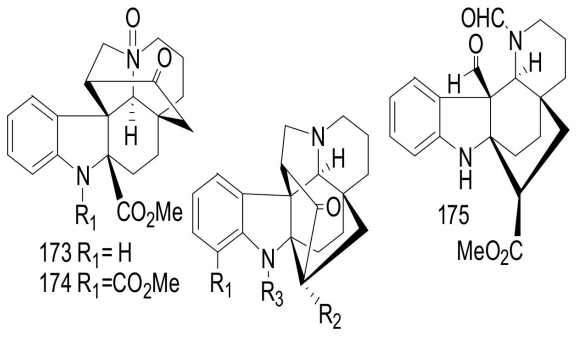

$179 \mathrm{R}_{1}=\mathrm{OMe}, \mathrm{R}_{2}=\mathrm{OH}, \mathrm{R}_{3}=\mathrm{H}$

$177 \mathrm{R}_{1}=\mathrm{O}, \mathrm{R}_{2}=\alpha-\mathrm{CO}_{2} \mathrm{Me} \quad 185 \mathrm{R}_{1}=\mathrm{OMe}, \mathrm{R}_{2}=\mathrm{OH}, \mathrm{R}_{3}=\mathrm{CO}_{2} \mathrm{Me}$ $178 \mathrm{R}_{1}=\mathrm{O}, \mathrm{R}_{2}=\beta-\mathrm{CO}_{2} \mathrm{H} \quad 186 \mathrm{R}_{1}=\mathrm{H}, \mathrm{R}_{2}=\mathrm{H}, \mathrm{R}_{3}=\mathrm{H}$ $183 \mathrm{R}_{1}=\mathrm{O}, \mathrm{R}_{2}=\beta-\mathrm{CO}_{2} \mathrm{Me}$ $184 \mathrm{R}_{1}=\mathrm{H}_{2}, \mathrm{R}_{2}=\beta-\mathrm{CO}_{2} \mathrm{H}$

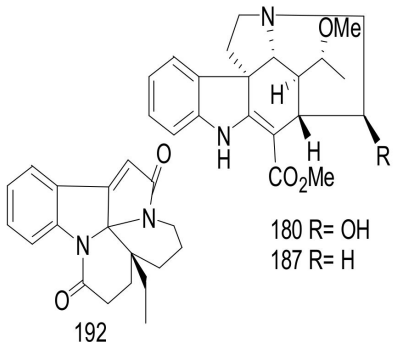

$\mathrm{HO}^{\prime \prime}>$
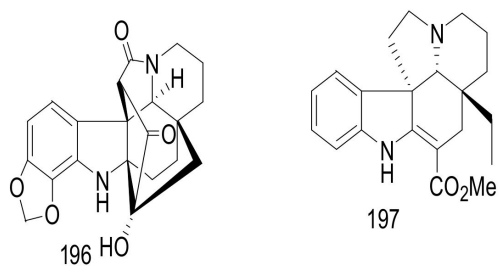

197

Figure 19. Compounds 171-197.

The vincamine-type compound $\mathbf{1 8 2}$ showed a potent inhibitory activity against HT29, HCT116, and A549 cancer cells, with $\mathrm{IC}_{50}$ values $=0.36,0.40$, and $0.51 \mu \mathrm{M}$, respectively. Meanwhile, compounds 188 and $\mathbf{1 8 9}$ showed moderate activities with $\mathrm{IC}_{50}$ values ranging from 2.00 to $2.61 \mu \mathrm{M}$ (Docetaxel, $\mathrm{IC}_{50}<0.0005 \mu \mathrm{M}$ ). These results indicated the structural features that are necessary for the presence of a vincamine-type carbonyl group at the C-16 position, forming an amide function group, and a methylene group or hydroxyl methine at C-19 position in 182, 188, and 189 [97]. The presence of a double bond in the piperidine ring between C-15 and C-16 may be responsible for increasing the activity of compound $\mathbf{1 8 2}$.

A study on the content of twigs of $K$. arborea grown in Thailand revealed the isolation of a new MIA, phutdonginin (191) [100], an eburnane-type compound, together with eight known compounds, among them, 164 [87], 189 [88] melodinine E (192) [101], kopsilongine (193), kopsamine (194) [94], (-)-methylenedioxy-11,12-kopsinaline (195) [87], decarbomethoxykopsiline (196) [102], and vincadifformine (197) [103]. Only 194 and 196 displayed AChE inhibition activity with MIR values 12.5 and $6.25 \mu \mathrm{g}$, respectively, compared with reference drug galanthamine MIR $=0.004 \mu \mathrm{g}$. In addition, compounds 194 and 198 also displayed the weak acetylcholinesterase (AChE) inhibition of $23.3 \%$ and $45.7 \%$ in a microplate test at $1 \mathrm{mM}$. Compounds 191 and 189 showed moderate inhibition of bacterium toward Escherichia coli TISTR 780 with MIC $=32 \mu \mathrm{g} / \mathrm{mL}$, with vancomycin and gentamycin references drugs with MIC values $0.125-0.25 \mu \mathrm{g} / \mathrm{mL}$ [100].

Malaysian Kopsia arborea was investigated and arboridinine (198) [85] was reported (Figure 20). The further investigation of the aerial parts of $K$. arborea led to the isolation of kopsiyunnanines J1 and J2 (199a and 199b) [104]. Compound 198 exhibited a moderate relaxation effect that was dependent on the contraction of phenylephrine-induced in the rat aortic rings, with an $\mathrm{EC}_{50}$ of $4.98 \mu \mathrm{M}$, and an $\mathrm{E}_{\max } 60.6 \pm 7.8 \%$ with the reference control isoprenaline with an $\mathrm{EC}_{50}$ value $=0.08 \mu \mathrm{M}$, and an $\mathrm{E}_{\max } 79.7 \pm 4.2 \%$ [85]. 


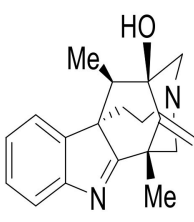

198

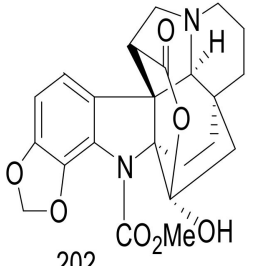

202<smiles>[R]C1([R2])C[C@]2(C(C)=O)CCCN3CCc4c(n1c1ccccc41)[C@]32C</smiles>

$207 \mathrm{R}_{1}=\mathrm{H}, \mathrm{R}_{2}=\mathrm{OH}$ $219 \mathrm{R}_{1}=\mathrm{OH}, \mathrm{R}_{2}=\mathrm{H}$

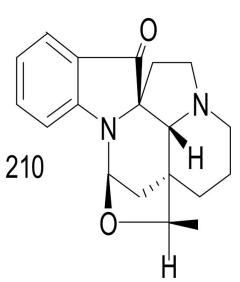

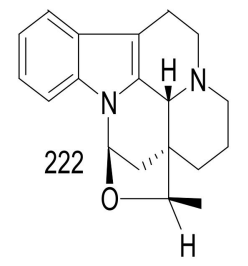

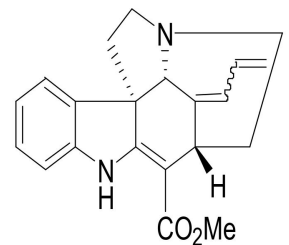

$19919 Z$

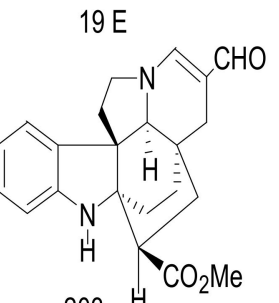
203

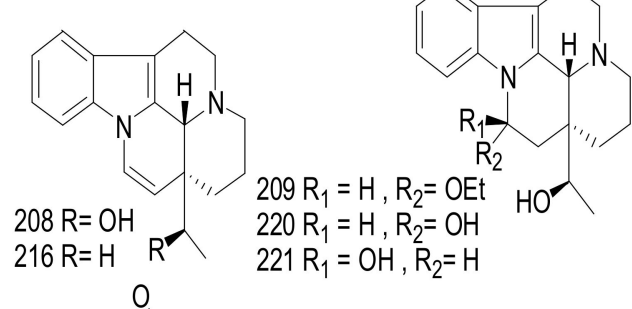

$\begin{array}{lllll}R_{1} & R_{2} & R_{3} & R_{4} & R_{5}\end{array}$ $211 \mathrm{OCH}_{2} \mathrm{O} \quad \mathrm{CO}_{2} \mathrm{Me} \mathrm{OH} \mathrm{OH}$ $212 \mathrm{OCH}_{2} \mathrm{O} \quad \mathrm{CO}_{2} \mathrm{Me} \mathrm{OH} \mathrm{H}$ $213 \mathrm{OMe} \mathrm{OMe} \mathrm{CO} 2 \mathrm{Me} \mathrm{OH} \mathrm{H}$

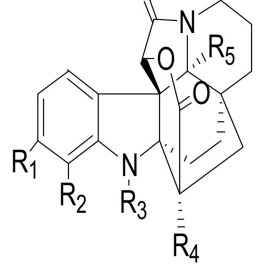

$215 R_{1}, R_{2}=0$ $217 \mathrm{R}_{1}=\mathrm{OH}, \mathrm{R}_{2}=\mathrm{H}$ $218 \mathrm{R}_{1}=\mathrm{H}, \mathrm{R}_{2}=\mathrm{OH}$ $204 R_{1}, R_{2}=0$ $205 R_{1}=R_{2}=H$

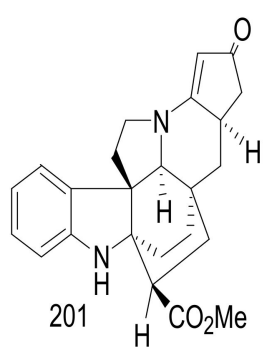

(1)

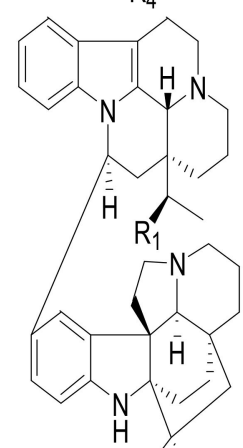

$223 \mathrm{R}_{1}=\mathrm{H}, \mathrm{R}_{2}=\mathrm{CO}_{2} \mathrm{Me}^{\mathrm{H}^{\prime}} \mathrm{R}_{2}$ $224 \mathrm{R}_{1}=\mathrm{OH}, \mathrm{R}_{2}=\mathrm{CO}_{2} \mathrm{Me}$

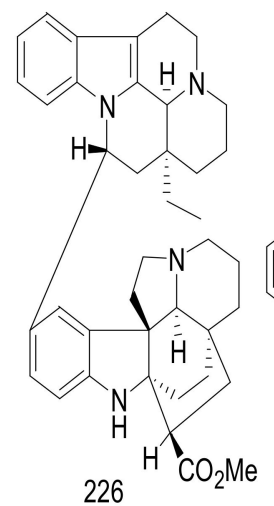
$225 \mathrm{R}_{1}=\mathrm{H}, \mathrm{R}_{2}=\mathrm{CO}_{2} \mathrm{H}$

Figure 20. Compounds 198-227.

Seven aspidofractinine -type alkaloids, paucidirinine (200), paucidirisine (201), paucidactinine (202), pauciduridine (203), paucidactine D (204), paucidactine E (205), and paucidisine (206), along with Additionally, four eburnane skeleton, (-)-19-oxoisoeburnamine (207), (-)-19(R)-hydroxyeburnamenine (208), (-)-19(R)-hydroxy-O-ethylisoeburnamine (209), and larutienine B (210) were isolated from Kopsia pauciflora [91]. Moreover, twelve compounds, paucidactine A (211), paucidactine B (212) [105], paucidactine C (213) [88], 5, 22dioxokopsane (214) [98], (+)-eburnamonine (215) [94], (+)-eburnamenine (216) [106], (-)eburnamine (217), (+)-isoeburnamine (218) [94], (+)-19-oxoeburnamine (219) [105], (-)-19(R)hydroxyisoeburnamine (220), (+)-19(R)-hydroxyeburnamine (221) [87], and larutienine A (222) [90] were published. Furthermore, three bisindole compounds have been identified, (-)norpleiomutine (223), (+)-kopsoffinol (224) [107], and (-)-demethylnorpleiomutine (225) [87] 
and (+)-kopsoffine (226) (Figure 20) [107], were identified from the same species. A bisindole alkaloid were isolated by Kitajima at et from Yunnan Kopsia arborea, named Kopsiyunnanine M (227) [108].

Compounds 223 and 224 exhibited growth inhibitory activity against MCF-7, PC-3, A549, and HCT-116, with $\mathrm{IC}_{50}$ values ranging between 11.5 and $25.1 \mu \mathrm{M}$ (Cisplatin, $\mathrm{IC}_{50}$ value in the range of 5.0-14.3 $\mathrm{MM}$ ). The obliteration of the biological activity in 225 may be due to the presence of a carboxylic group in C-16, instead of a methoxycarbonyl group in 223 [91]. Arborisidine (228) and arbornamine (229) were isolated from a Malaysian $K$. arborea. Compound 228 represented a unique skeleton [109]. Compounds 228 and 229 exhibited no activities against KB, PC-3, HCT116, A549 and HT-29 cells [109].

Six new Kopsinidine C-E (230-232), 11,12-methylenedioxychanofruticosinic acid (233), 12-methoxychanofruticosinic acid (234), and N(4)-methylkopsininate (235), in addition to chanofruticosinic acid (236) as new natural compound [110], along with compounds 163, 164, 178, 183, 179, and 215 (Figure 21) were isolated from K. officinalis. Additionally, Kopsinine methochloride (237), demethoxycarbonylkopsin (238) [111], methyl chanofruticosinate (239), methyl 11,12-methylenedioxychanofruticosinate (240) [94], methyl 12-methoxychanofruticosi nate (241), methyl 11,12-methylenedioxy- $N_{1}$-decarbomethoxychanofruticosinate (242) [112], kopsininic acid (243), and (-)-11,12-methylenedioxykopsinaline (244) [98] were identified from the same species. Furthermore, (-)-N-methoxycarbonyl-11,12-methylenedioxykopsinaline (245) [98], (-)-N-methoxycarbonyl- 12-methoxykopsinaline (246), N-carbomethoxy-11-hyd roxy-12- methoxykopsinaline (247) [113], kopsinoline (248) [114], (-)-12-methoxykopsinaline (249) [98], 11,12-methylenedioxykopsinaline N(4)- oxide (250) [87], kopsinine B (251) [115], rhazinilam (252) [66], and pleiocarpamine methochloride (253) [116] were all isolated from the twigs and leaves of chines K. officinalis. Compound 252 displayed a significantly inhibition effect of the human $T$ cell proliferation, which was activated by using anti-CD3/anti-CD28 antibodies, with an $\mathrm{IC}_{50}=1.0 \mu \mathrm{M}$, showing stimulation, with an $\mathrm{IC}_{50}=1.1 \mu \mathrm{M}$ [110]. Compound 252 was indicated to have the highest cytotoxic effect due to the presence of a hydroxyl group in C-14 and C-15 position [110].

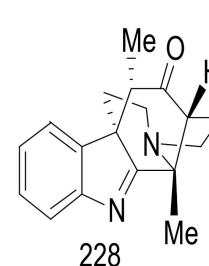

228<smiles></smiles>

229

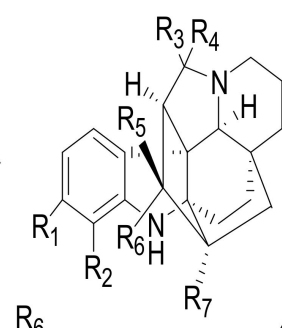

$\begin{array}{lllllll}R_{1} & R_{2} & R_{3} & R_{4} & R_{5} & R_{6} & R_{7}\end{array}$ $230 \mathrm{H} \quad \mathrm{H} \mathrm{H}$ OH H OH $231 \mathrm{H} \quad \mathrm{OMeH} \mathrm{H} \mathrm{OH} \mathrm{H} \mathrm{OH}$ $232 \mathrm{OCH}_{2} \mathrm{O} \quad \mathrm{H}$ H OH H OH $238 \mathrm{H} \mathrm{H} \mathrm{H} \mathrm{H} \mathrm{O} \mathrm{OH}$

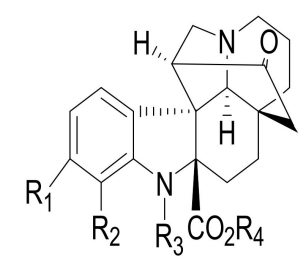

$\begin{array}{llll}R_{1} & R_{2} & R_{3} & R_{4}\end{array}$ $233 \mathrm{OCH}_{2} \mathrm{O} \quad \mathrm{CO}_{2} \mathrm{Me} \mathrm{H}$ $234 \mathrm{H} \quad \mathrm{OMe} \mathrm{CO}_{2} \mathrm{Me} \mathrm{H}$ $236 \mathrm{H} \quad \mathrm{H} \quad \mathrm{CO}_{2} \mathrm{Me} \mathrm{H}$ $239 \mathrm{H} \quad \mathrm{H} \quad \mathrm{CO}_{2} \mathrm{Me} \mathrm{Me}$ $240 \mathrm{OCH}_{2} \mathrm{O} \quad \mathrm{CO}_{2} \mathrm{Me} \mathrm{Me}$ $241 \mathrm{H} \quad \mathrm{OMe} \mathrm{CO}_{2} \mathrm{Me} \mathrm{Me}$ $242 \mathrm{OCH}_{2} \mathrm{O} \quad \mathrm{H} \quad \mathrm{Me}$

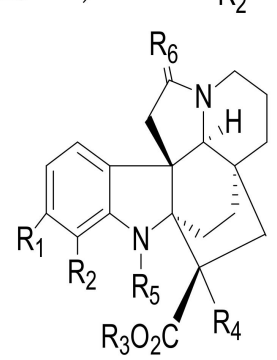<smiles></smiles>

$\begin{array}{lllll}R_{1} & R_{2} & R_{3} & R_{4} & R_{5}\end{array}$ $243 \mathrm{H} H \mathrm{H} H \mathrm{H}$ $244 \mathrm{OCH}_{2} \mathrm{O} \quad \mathrm{MeOH} \mathrm{H}$ $245 \mathrm{OCH}_{2} \mathrm{O} \quad \mathrm{MeOH} \mathrm{CO}_{2} \mathrm{Me} \mathrm{H}$ $246 \mathrm{H} O \mathrm{OMe} \mathrm{MeOHCO} 2 \mathrm{Me} \mathrm{H}, \mathrm{H}$ $2470 \mathrm{H} \quad \mathrm{OMe} \mathrm{Me} \mathrm{OH} \mathrm{CO} 2 \mathrm{Me} \mathrm{H}, \mathrm{H}$ $248 \mathrm{H} \quad \mathrm{H} \quad \mathrm{Me} \mathrm{H} \quad \mathrm{H}, \mathrm{HN}(4) \rightarrow 0$ $249 \mathrm{H} \quad \mathrm{OMe} \mathrm{MeOH} \mathrm{H}$ $250 \mathrm{OCH}_{2} \mathrm{O} \quad \mathrm{MeOH} \mathrm{H}$ $251 \mathrm{H} \mathrm{H} \quad \mathrm{MeOH} \mathrm{H}$
$\mathrm{H}, \mathrm{H}$ $\mathrm{H}, \mathrm{H} \mathrm{N}(4) \rightarrow \mathrm{O}$ $\mathrm{H}, \mathrm{H}$

Figure 21. Compounds 228-253. 
Kopsioffines A-C (254-256) [117] (Figure 22) were isolated from the leaves and stems of K. officinalis. These compounds possess a relatively novel ten-membered lactam ring [117]. Additionally, five MIAs, Kopsifolines G-K (257-261) were identified from the same plant [118]. Moreover, kopsifoline A (262) was isolated from the aerial parts of an unidentified Kopsia sp. [119]. Compounds 259-261 exhibited cytotoxic effects against dermatoma (HS-1, A431, SCL-1, HS-4), gastric carcinoma (BGC-823), breast cancer (MCF-7), and colon cancer (SW480), with $\mathrm{IC}_{50}$ values in a range between 11.8 and 13.8; between 10.3 and 12.5; between 7.3 and $9.5 \mu \mathrm{M}$, respectively (Adriamycin, $\mathrm{IC}_{50}<34 \mathrm{nM}$ ). Compound 261 showed a potent cytotoxic effect that may be due to the presence of two hydroxyl groups in the C-14 and C-15 positions, instead of one hydroxyl group at C-15 position in compounds 259 and 260. Compounds 257, 258 and 262 exhibited a weak cytotoxic effect with $\mathrm{IC}_{50}$ values $>20 \mu \mathrm{M}$. This may be due to the absence of a hydroxyl group in that position [118]. Compounds 254-256 exhibited weak inhibitory effects on yeast $\alpha$-glucosidase in vitro with $\mathrm{IC}_{50}$ values $>50 \mu \mathrm{M}$ [118]. Compounds 259-260 exhibited interesting antifungal and antimicrobial activities toward five pathogen bacteria Escherichia coli, Pseudomonas aeruginosa, Enterobacter cloacae, Shigella dysenteriae and Klebsiella pneumoniae), and also exhibited an antibacterial effect on the oral pathogens Streptococcus viridans and Streptococcus mutans. Netilmicin was used as a reference drug, with MIC values $<0.18 \mathrm{~mm}$. 5-Flucytocine was used as a positive control with MIC values $<0.09 \mathrm{mM}$. Alkaloid 261 displayed the highest antimicrobial activity toward the tested pathogens, with an MIC value of $0.15-1.14 \mathrm{mM}$, while compounds 259 and 260 showed significant activities, with MIC values of 0.77-3.09 and 0.72-1.37 mM. Compounds 257, 258 and 262 were inactive. The present of a hydroxyl group at the piperidine ring enhanced the anticancer and antimicrobial activity in this subtype of indoles [118]. The investigation of the aerial parts of $K$. arborea led to the isolation of three compounds: kopsiarborines A-C (263-265) [120]. Meanwhile, the study of the aerial parts of $K$. officinalis led to the reporting of three MIAs, kopsiaofficines A-C (266-268) (Figure 22) [121]. Compounds 263 and 264 showed significant cytotoxic activities against H446, H292, A549, H460, ATCC, and 95-D, with $\mathrm{IC}_{50}$ values $<20 \mu \mathrm{M}$, (Doxorubicin, $\mathrm{IC}_{50}$ value $=0.06 \mu \mathrm{M}$ ). Compound 264 exhibited a potent activity with $\mathrm{IC}_{50}$ values $<9.5 \mu \mathrm{M}$, and compound 265 was inactive [120]. Compound 268 exhibited a potent cytotoxicity against H446, A549, ATCC, 95-D, H460, H292, SPCA-1, and lung cancer cells, with $\mathrm{IC}_{50}$ values $<10 \mathrm{mM}$, while compound 266 showed some cytotoxic activity with $\mathrm{IC}_{50}$ value $<20 \mu \mathrm{M}$ (Doxorubicin, $\mathrm{IC}_{50}=13.7-33.7 \mathrm{nM}$ ) [121].

Kopsiofficines H-L (269-273) [122] (Figure 23), together with fourteen compounds, 164, 208, 239, 241, (+)-O-methyleburnamine (274) [93], (-)-O-methylisoeburnamine (275) [123], 16isoeburnamine (276) [124], 20-oxoeburnamenine (277) [125], methyl 11, 12-methylenedioxyc hanofruticosinate (278) [99], methyl N-(decarbomethoxy)-11, 12-(methylenedioxy) chanofruticosinate (279) [126], O-methylleuconolamm (280) [127], leuconodine D (281) [128], oxayohimban-16-carboxylic acid (282) [129], and 19, 20-dihydroisositsirikine (283) [130] (Figure 23), were identified from the stems of K. officinalis plant [122]. Compounds 164, 241, 270, 271, 274, 275, 279, and 281 exhibited significant anti-inflammatory activity towards IL-1 $\beta$, PGE2 and TNF- $\alpha$ at $5 \mu \mathrm{g} / \mathrm{mL}$. Deametasona was used as a positive control at $10 \mu \mathrm{g} / \mathrm{mL}[122]$. 

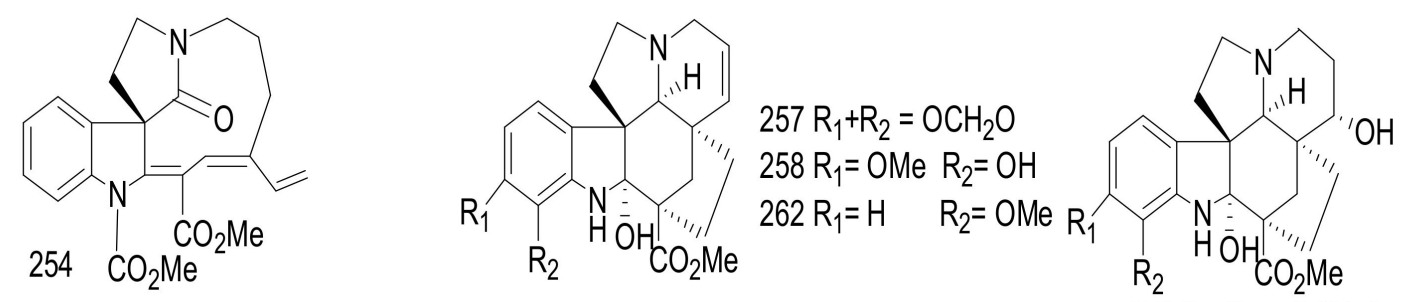

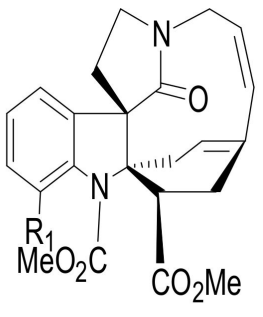

$255 \mathrm{R}_{1}=\mathrm{H}$

$256 \mathrm{R}_{1}=\mathrm{OH}$

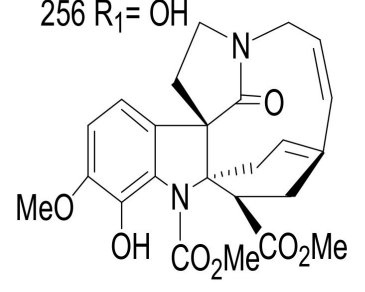

265

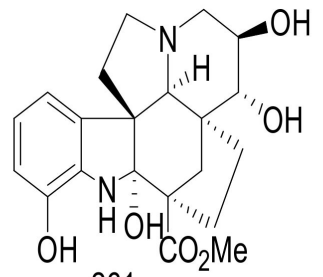

261

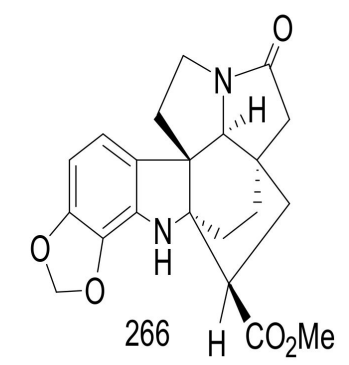

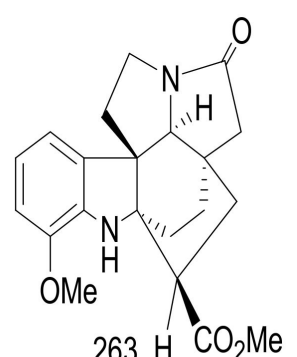

$263 \mathrm{H} \mathrm{CO}_{2} \mathrm{Me}$

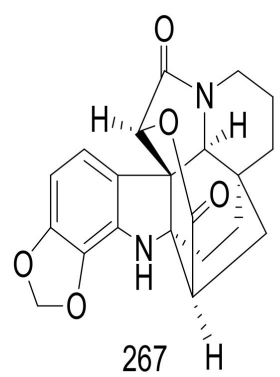

$259 \mathrm{R}_{1}+\mathrm{R}_{2}=\mathrm{OCH}_{2} \mathrm{O}$

$260 \mathrm{R}_{1}=\mathrm{OMe} \mathrm{R}_{2}=\mathrm{OH}$
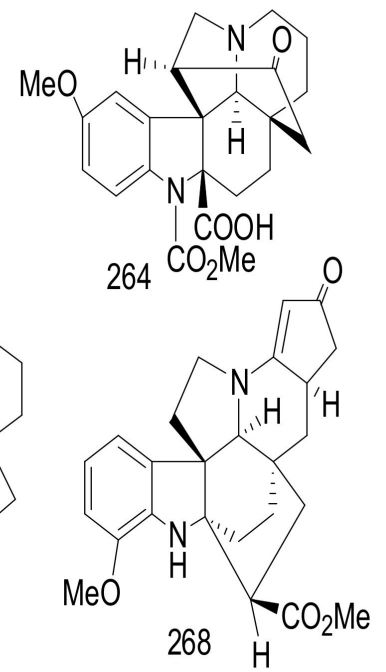

Figure 22. Compounds 254-268.
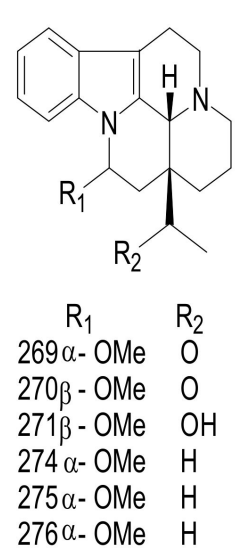
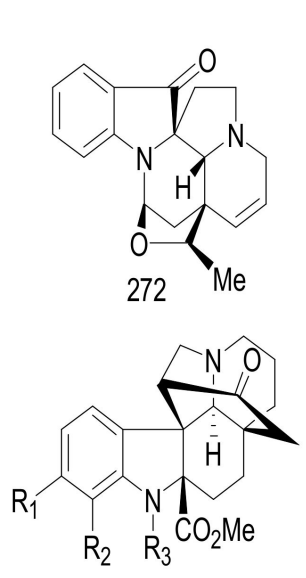

$$
\begin{array}{rrr}
\mathrm{R}_{1} & \mathrm{R}_{2} & \mathrm{R}_{3} \\
278 \mathrm{H} & \mathrm{H} & \mathrm{H} \\
2790 \mathrm{OCH}_{2} \mathrm{O} & \mathrm{H}
\end{array}
$$
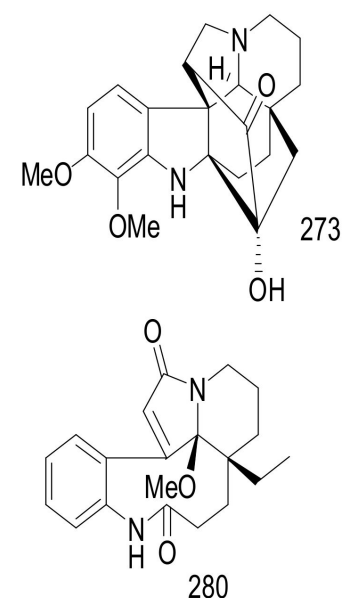

280

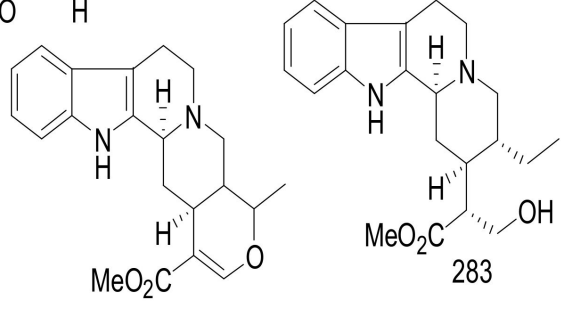

282

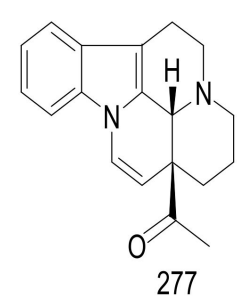

277

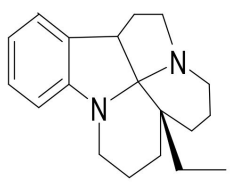

281

Figure 23. Compounds 269-283. 
Table 1 methyl chanofruticosinate-type (164, 173-175), aspidosoermatan-type (169, 199), kopsine-type $(\mathbf{1 7 9}, \mathbf{1 8 5}-\mathbf{1 8 6})$, strychnos-type $(\mathbf{1 8 0}, \mathbf{1 8 7})$, vincamine-type $(\mathbf{1 8 1}, \mathbf{1 8 8}, \mathbf{1 8 9})$, paucidactine-type $(\mathbf{2 0 4}, \mathbf{2 0 5})$ and eburnane-type (207-210), all these subtypes belongs to the main class aspidospirma, and very few compounds belong to the main class of corynanthetype indoles (Figure 4). Vincamine and methyl chanofruticosinate derivatives showed interesting biological activity.

\section{Rauvolfia}

Rauvolfia (family Apocynaceae) contains 60 species. It contains trees or shrubs that are distributed in Africa, America, and Asia [131]. Rauvolfia serpentine is one of the most important medicinal plant that has been considered as a drug lead for a long time [132]. Rauvolfia has been used traditionally for the treatment of several diseases, such as high blood pressure (hypertensive), fever (malaria), arrhythmia, cancer, oxidative stress, microbial problems, intestinal spleen ailments, and various mental disorders [133]. Therapeutically, it is a source of monoterpenoid indoles, including ajmaline (antiarrhythmic), ajmalicine, yohimbine, reserpine (antihypertensive), and serpentine [133].

A review entitled "Rauvolfia serpentina L. Benth. ex Kurz. phytochemical, pharmacological and therapeutic aspects" was published in 2013 and evaluated various bioactive compounds as ajmaline, ajmalicine, deserpidine, reserpine, reserpiline, serpentine, rescinnamine and yohimbine [132]. A review entitled "Chemical and Biological Perspectives of Monoterpene Indole Compounds from Rauwolfia species" mentioned the compounds obtained until 2016 [134]. Another review described the structures and pharmacological potentials of the plant species Rauvolfia tetraphylla L. (Apocynaceae) [135].

Two normonoterpenoid indole compounds were isolated from the aerial parts of Rauvolfia vomitoria, rauvomines A (284) and B (285) [136] along with two known compounds peraksine (286) (Figure 24) [137] and alstoyunine A (287) [42]. Compound 285 displayed significant anti-inflammatory effects against murine macrophages (RAW 264.7), with an $\mathrm{IC}_{50}$ value $=39.6 \mu \mathrm{M}$, whereas, compounds 284, 286 and 287 displayed moderate antiinflammatory effects with $\mathrm{IC}_{50}$ values $=55.5,65.2$, and $75.3 \mu \mathrm{M}$, respectively, (Celecoxib, $\mathrm{IC}_{50}=34.3 \mu \mathrm{M}$ ) [136]. Compound 285 showed a potent activity which maybe double the number of connections linking C-20 to C-16 in sarpagine-type indoles, compared with compound 284 [63].

Three compounds, 11-hydroxyburnamine (288) and rauvoyunnanines A and B (289290) were identified from Chinese $R$. yunnanensis [138]. Additionally, fourteen compounds 135 [139], lochnerine (291) [140], serpentinic acid (292) [141], reserpine (293) [142], (-)yohimbine (294) [143], ajmaline (295) [143], mauiensine (296) [144], ajmalicine (297) [145], sitsirikine (298) [146], strictosidinic acid (299) [147], caboxine B (300) [148], isocaboxine B (301) [148], spegatrine (302) [149], and 19(S),20(R)-dihydroperaksine (303) [150] (Figure 24) were isolated also from chines $R$. yunnanensis. Compound 293 displayed a weak cytotoxicity against HT-29 and SW480, with $\mathrm{IC}_{50}$ values $=35.2$ and $45.3 \mu \mathrm{M}$, respectively. Auranofin was used as a positive control and showed cytotoxicity with $\mathrm{IC}_{50}$ values $=2.5$ and $3.9 \mu \mathrm{M}$, respectively. Compounds 294 and 299 displayed immunosuppressive activities on human T cell proliferation, with $\mathrm{IC}_{50}$ values $=5.9$ and $5.0 \mu \mathrm{M}$, respectively. All compounds except 294 and 299 showed weak activities with $\mathrm{IC}_{50}$ values $>50 \mu \mathrm{M}$ [138]. The metabolites were identified from genus Rauvolfia and were categorized under the corynanthe-type. The compounds were also classified under three subclasses including: sarpagine-type 284-285, picraline-type 288 and ajmaline-type 295-296 and 298 [138]. 


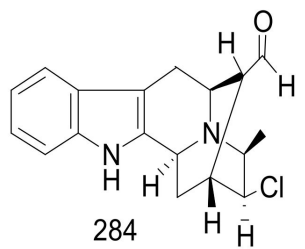

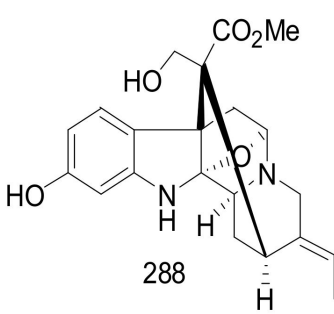

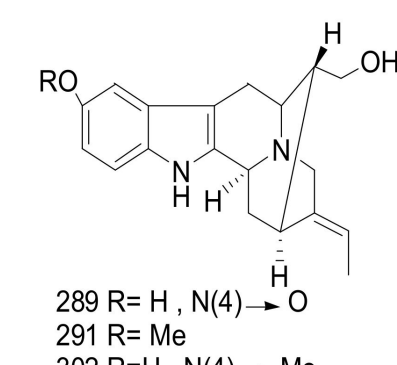

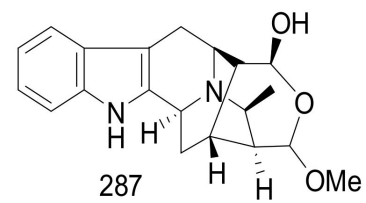<smiles>C[C@H]1OC=C(C(=O)O)[C@@H]2Cc3c([nH]c4ccccc34)[C@@H]1C2</smiles>

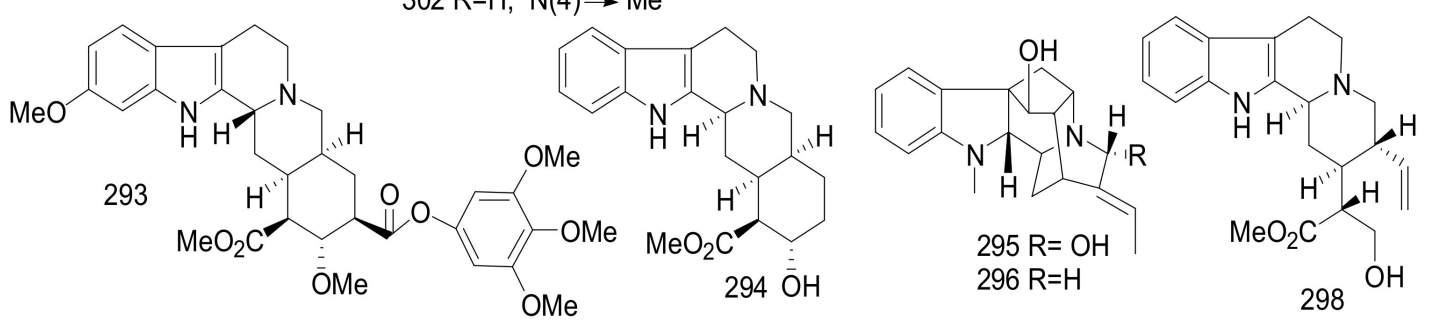<smiles>COC(=O)[C@H]1C=CO[C@H](C)[C@H]1CN1CCc2c([nH]c3ccccc23)[C@H]1C</smiles><smiles>C=C[C@H]1[C@H](OC(C)=O)OC=C(C(=O)O)[C@H]1C[C@@H]1NCCc2c1[nH]c1ccccc21</smiles><smiles>C/C=C(/C)OC</smiles>

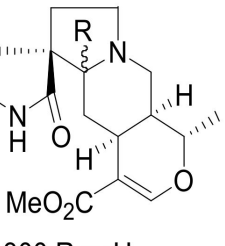

$300 \mathrm{R}=\alpha \mathrm{H}$ $301 \mathrm{R}=\beta \mathrm{H}$<smiles>C[C@@H]1[C@@H]2Cc3[nH]c4ccccc4c3C[C@H](C2CO)[C@H]1CO</smiles>

$303 \overline{\bar{H}}$

Figure 24. Compounds 284-303.

\section{Ervatamia}

The genus Ervatamia contains 120 species. It is distributed in Asia and Australian. Of which, fifteen species and five varieties are grown in south China. Ervatamia is a rich source of iboga-type MIAs, which is characterized by structural novelty and biological diversity including neuroprotective, anti-tumor, and anti-addiction activities [151-153].

Six Iboga-type compounds: ervataine (304) [151], ibogaine (305) [154], coronaridine (306) [49], heyneanine (307) [155], voacangine hydroxyindolenine (308) [156,157] and coronaridine hydroxyindolenine (309) [158,159] (Figure 25), were obtained from the Chinese Ervatamia yunnanensis [151].

Compound 306 exhibited significant protective effects toward $\mathrm{MPP}^{+}$(1-methyl-4phenylpyridinium) and induced damage in primary cortical neurons with an $\mathrm{IC}_{50}=$ $12.5 \mu \mathrm{M}$. Parkinson's disease (PD) is caused by $\mathrm{MPP}^{+}$a toxic agent that interferes with the function of mitochondria, thus causing neuronal damage and death. Brain-derived neurotrophic factor (BDNF) was used as a positive control and showed an inhibitory effect, with an $\mathrm{IC}_{50}$ value $=200 \mathrm{ng} / \mathrm{mL}[49]$.

Eight compounds, coronaridine (306) [49], coronaridine hydroxyindolenine (309) [158,159], 10-hydroxycoronaridine (310) [160], voacangine (311) [153], 19(S)-heyneanine (312) [160], 19(R)-heyneanine (313) [161], heyneanine hydroxyindolenine (314) [162], and vobasine (315) [163], were identified from the stems of E. hainanensis. Compounds 306, 309-315 displayed acetylcholinesterase inhibitory activities. Compounds 306 and 311 displayed a potent cholinesterase inhibitory effect, with $\mathrm{IC}_{50}$ values $=8.6$ and $4.4 \mu \mathrm{M}$, respectively. Galan- 
tamine was used as a reference drug, with an $\mathrm{IC}_{50}=3.2 \mu \mathrm{M}$, that is used for Alzheimer's disease [164]. Compound 310 possessed a hydroxyl group at the phenyl moiety, which was replaced by proton in compound 306. This led to a decrease in the inhibitory activity of AChE in 306 compared to 310. The methoxy group at the phenyl moiety in 311, led to an improvement in the activity. This indicated that the electron-donor substituents attached at the phenyl group were important for the improvement of AChE inhibition [164].

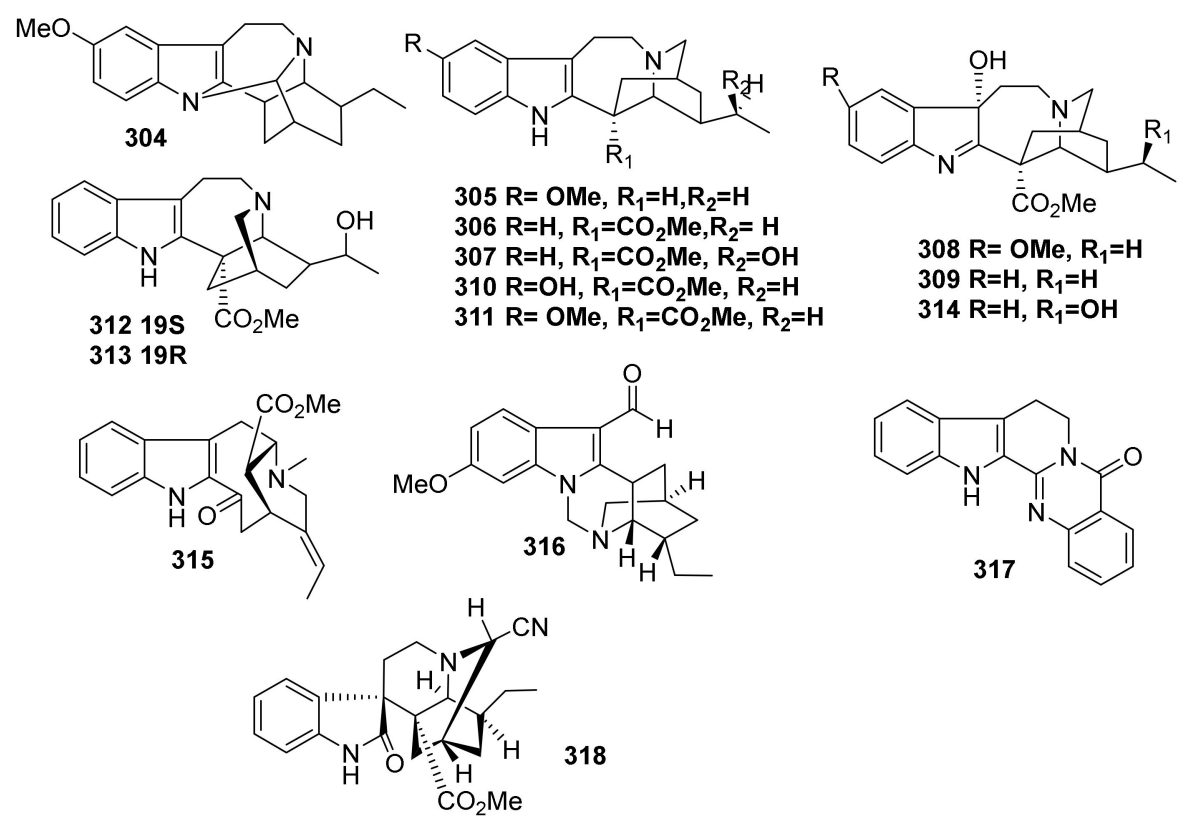

Figure 25. Compounds 304-318.

Ervachinine E (316) [165] and rutaecarpine (317) [166] were isolated from E. chinensis [165]. It displayed moderate antitumor activities against HL-60, SMMC-7721, A-549, and SW480 cancer cells, with values of $\mathrm{IC}_{50}$ ranging between 6.59 and $14.70 \mu \mathrm{M}$. (Cisplatin, $\mathrm{IC}_{50}$ values between 1.00 and $\left.26.75 \mu \mathrm{M}\right)$ [165].

The compound Ervahainine A (318), an oxindole derivative that is cyano-substituted, was identified from the twigs and leaves of E. hainanensis [167]. Compound 318 showed growth inhibitory activities toward HepG2 and HepG2/ADM cells with $\mathrm{IC}_{50}$ values of $12.47 \pm 0.24$ and $17.68 \pm 0.31 \mu \mathrm{M}$ [167].

Seven new iboga-type derivatives: ervaoffines A-D (319-322), (7S)-3-oxoibogaine hydroxyindolenine (323), ibogaine-5,6-dione (324), and 19-epi-5-oxovoacristine (325), along with ten compounds, 305, 307, 311, iboluteine (326) [168], (7S)- ibogaine hydroxyindolenine (327) [157], ibogaline (328) [169], conopharyngine (329) [170], voacristine (330) [171], 19Shydroxyibogamine (331) [172], and ibogaine $N_{4}$-oxide (332) $[173,174]$ (Figure 26), were isolated from the twigs and leaves of E. officinalis.

Seven compounds, 3-oxo-7R-coronaridine hydroxyindolenine (333), 3S-cyano-7Scoronaridine hydroxyindolenine (334), 3R-hydroxy-7S-coronaridine hydroxyindolenine (335), 3S-(24S-hydroxyethyl)-coronaridine (336), 3S-(24R-hydroxyethyl)-coronaridine (337), 5-oxo-6S-hydroxycoronaridine (338) and 5-oxo-6S-methoxy-coronaridine (339) [175], along with six others, 306, 7S-coronaridine hydroxyindolenine (340) [176], 3-oxo-7S-coronaridine hydroxylindolenine (341) [177], 5-oxocoronaridine (342) [177], 3-oxocoronaridine (343) [178] and pseudoindoxyl coronaridine (344) [177], (Figure 27) from identified from twigs and leaves of E. hainanensis [175]. 

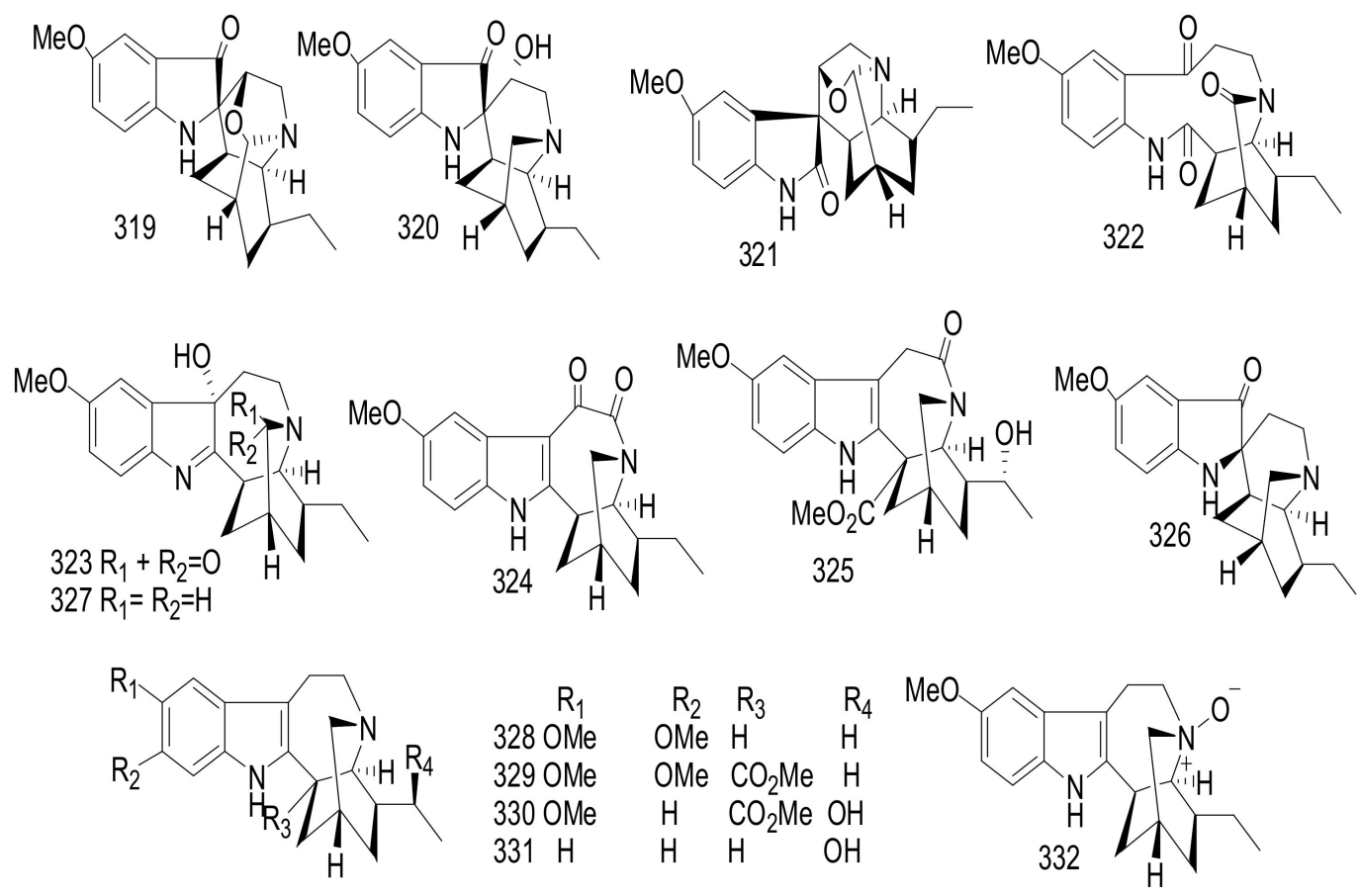

Figure 26. Compounds 319-332.

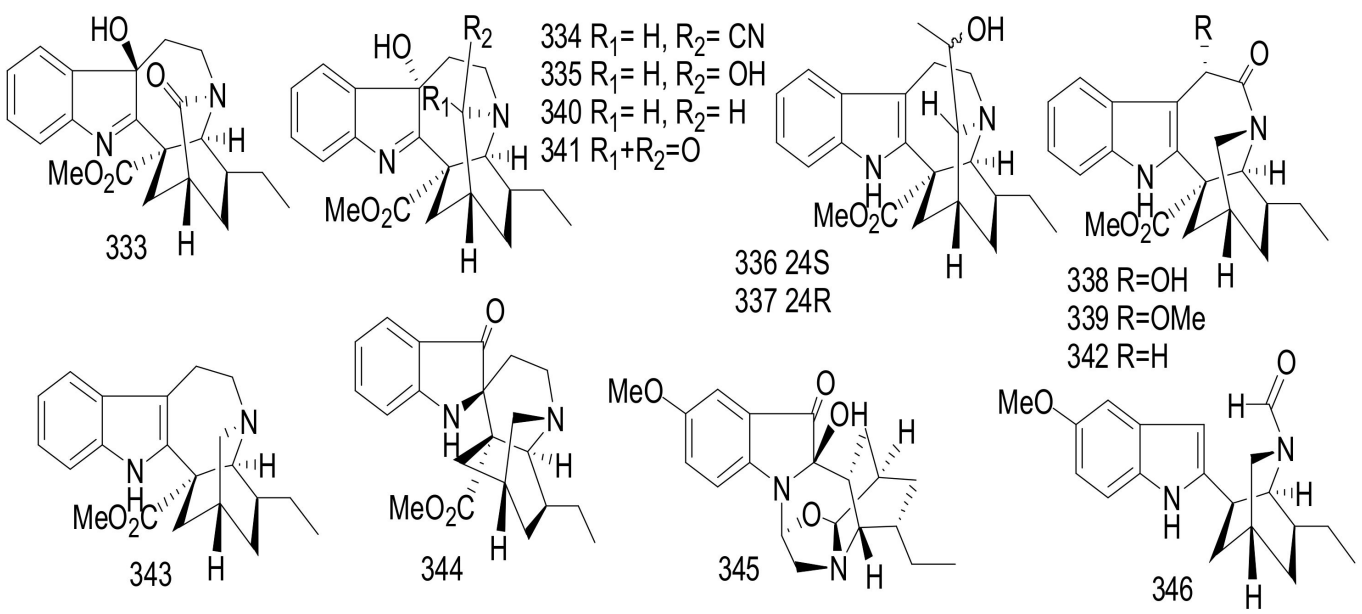

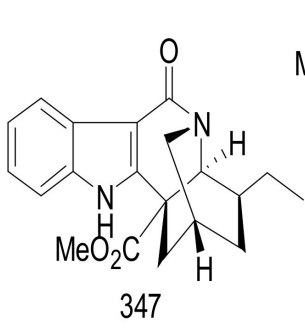

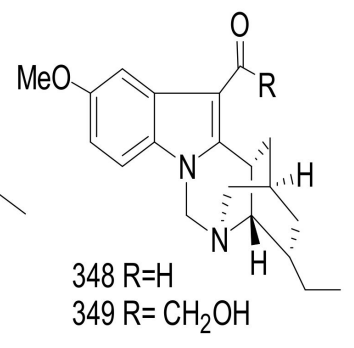

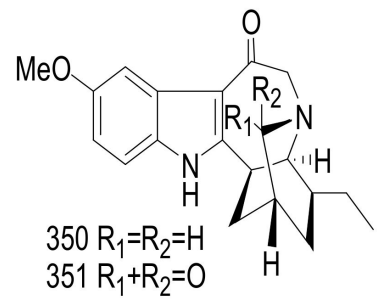

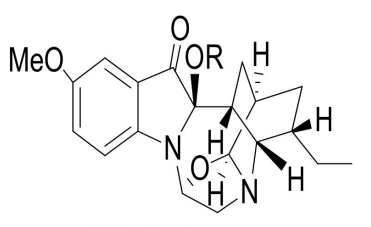

$352 \mathrm{R}=\mathrm{H}$ $353 \mathrm{R}=\mathrm{Me}$

Figure 27. Compounds 333-353.

Another study on the twigs and leaves of E. officinalis led to the reporting of three MIAs, ervaoffines E-G (345-347) [179], and six compounds 306, 342, lirofoline A (348), lirofoline B (349) [172], 6-oxo-ibogaine (350) [180], and 8-oxo-ibogaine lactam (351) [179-181]. Compound 347 showed a significant neuroprotective effect towards damage induced by oxygen-glucose deprivation (OGD) of the cortical neurons cultured of ischemic stroke 
in vitro, with an $\mathrm{IC}_{50}=100 \mu \mathrm{M}$, Neuroserpin was used as a reference drug, with an $\mathrm{IC}_{50}=$ $20 \mathrm{ng} / \mathrm{mL}$ [179]. Two compounds were obtained from the roots of E. chinensis, erchinines $A$ and B $(352,353)$ [63]. Both compounds 352 and 353 displayed a potent significant antibacterial activity toward Bacillus subtilis which was better than that of the antibacterial drugs fibraurtine with an MIC $=25 \mu \mathrm{M}$ and berberine with an MIC $=12.5 \mu \mathrm{M}$ that are derived from plant. Additionally, compound 352 displayed an equal antifungal effect against (Trichophyton rubrum) to the reference drug griseofulvin, with an MIC $=6.25 \mu \mathrm{M}$.

Ervapandine A (354) [182], 3R-hydroxyibogaine (355) [182], and 12-hydroxyakuammicine $\mathrm{N}_{4}$-oxide (356) [182], along with four known ones, 313, 305, 19-epi-voacristine (357) [183], taberdivarine I (358) [184] and 12-hydroxyakuamicine (359) [185], (Figure 28) were identified from the leaves and twigs of Chinese E. pandacaqui [182].

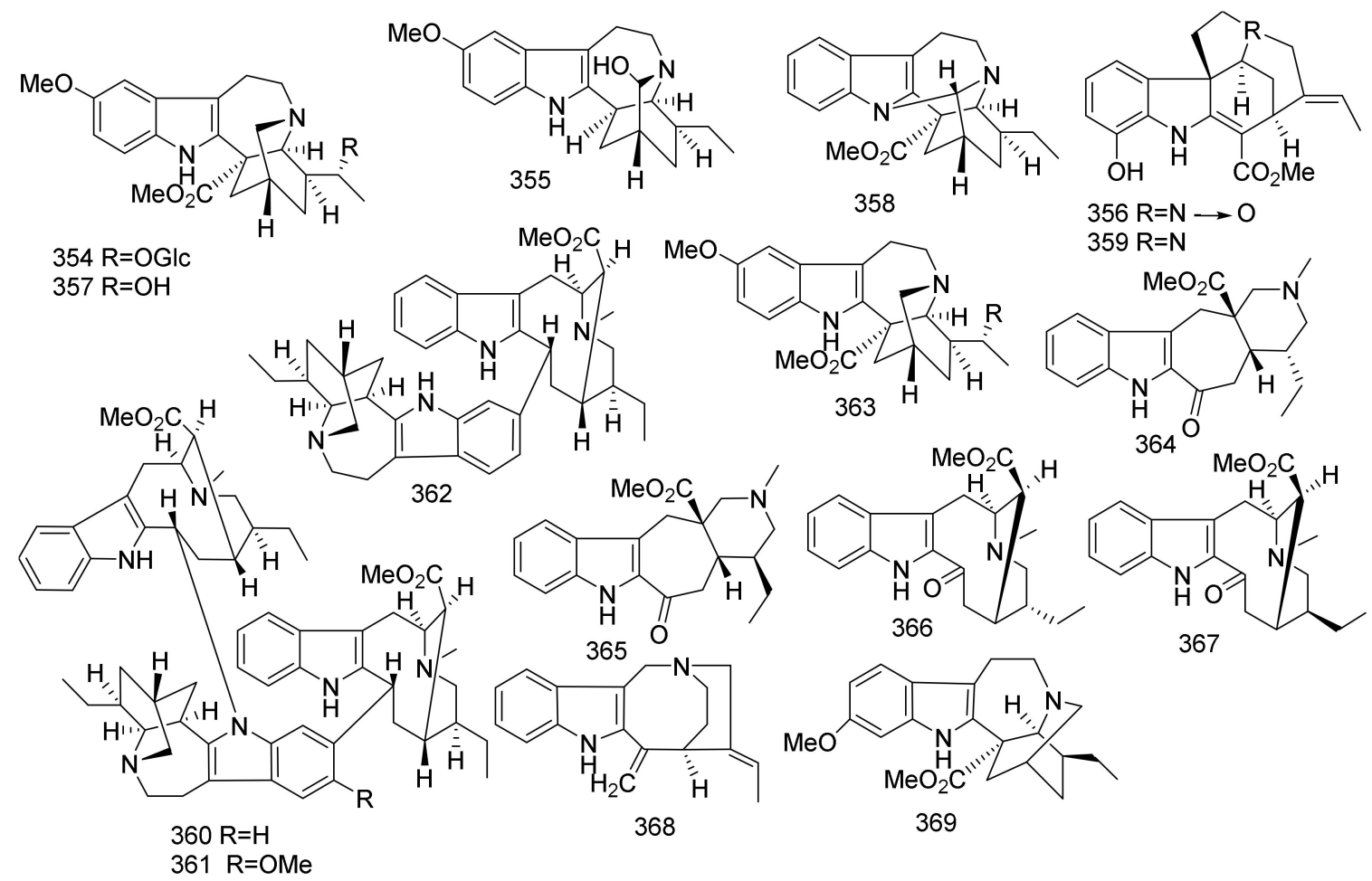

Figure 28. Compounds 354-369.

Liu et al. (2018) [186] studied the roots of E. divaricate and identified two unprecedented trimeric MIAs, Ervadivamines A (360) and B (361), together with the dimeric compound, 19,20-dihydroervahanine A (362), (Figure 29) and two monomeric ones, ibogaine (305) and Ibogamine (363) [187]. Compound 359 displayed a moderate cytotoxic effect against MCF-7, with an $\mathrm{IC}_{50}$ value $=33.61 \mu \mathrm{M}$ [182]. Compound 360 showed a significant positive cytotoxicity against MCF-7, A-549, HT-29 and HepG2/ADM and showed potent effect against $\mathrm{HepG} 2 / \mathrm{ADM}$, with an $\mathrm{IC}_{50}$ value $=12.55 \pm 0.54 \mu \mathrm{M}$ (Adriamycin, $\left.\mathrm{IC}_{50}=45.70 \pm 2.15 \mu \mathrm{M}\right)[186]$.

Two pair of MIAs epimers composed of, ervatamine (364), [188] 20-epi-ervatamine (365), [188] dregamine (366), and [188] tabernaemontanine (367) [188] and two compounds, apparicine (368) [189] and isovoacangine (369) [190], were isolated from E. yunnanensis [191].

The Ervatamia genus is known to produce iboga-type indole derivatives, which contain two subclasses, flabelliformide-type $(364,365)$ and apparicine-type (368) (Figure 28), with compounds belongonging to the main class corynathe. The iboga-type showed an interesting bioactivity in the nervous system. 


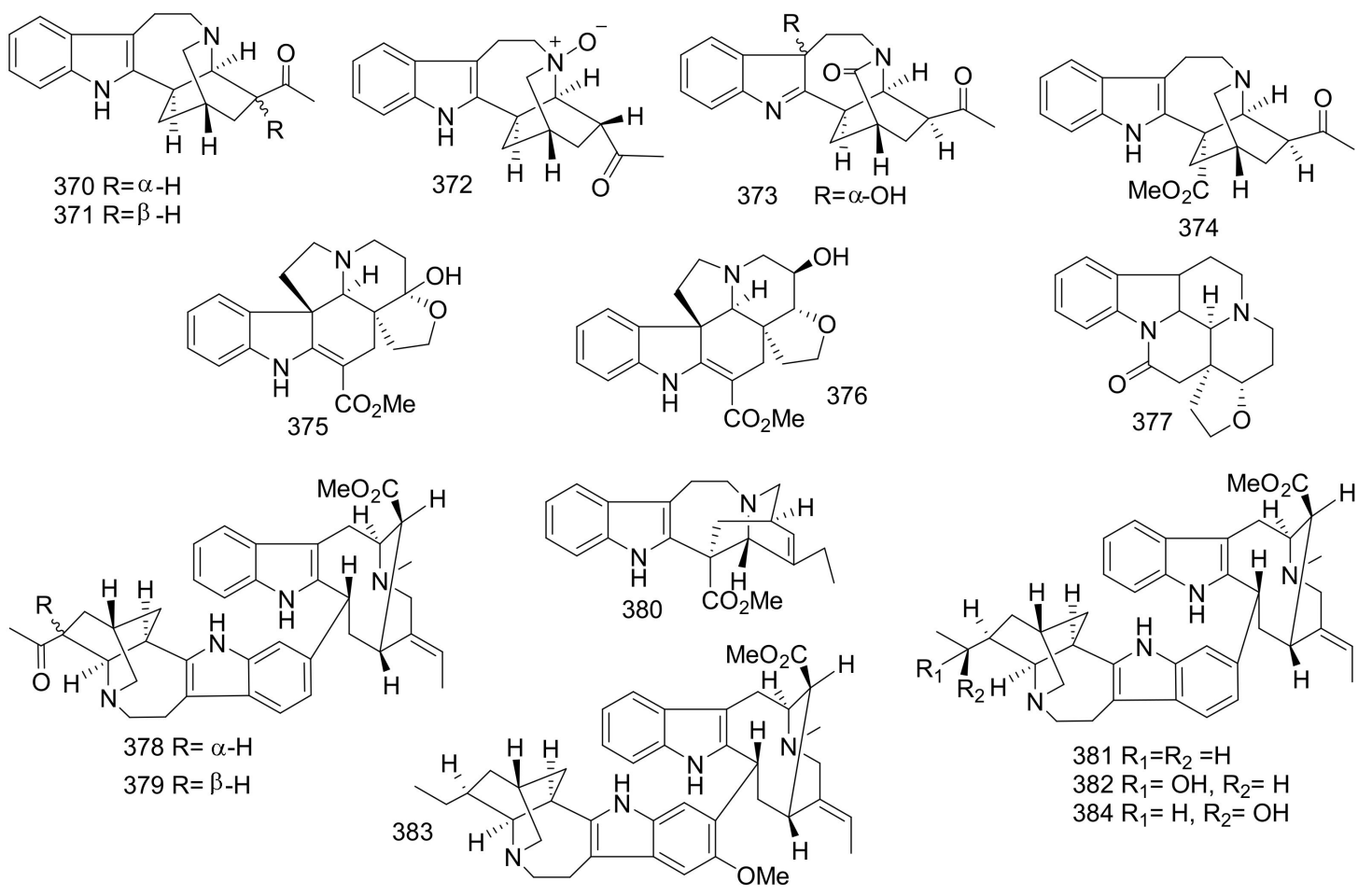

Figure 29. Compounds 370-384.

\section{Tabernaemontana}

The Genus Tabernaemontana (subfamily Rauvolfioideae) contains 110 species, which are distributed throughout tropical and subtropical regions. Thirty species are grown in Brazil, whereas, 44 species were grown in America and the rest in different places around the world. Traditionally, the plants of this genus have been used for the treatment of hypertension, sore throat, and abdominal pain [6,192]. A review article entitled "Brazilian Tabernaemontana genus: indole compounds and phytochemical activities" activities was published in 2016 [6]. It concerned in the monomeric and dimeric MIAs reported from the genus. A review article entitled: A review on tabernaemontana spp.: Multipotential medicinal plant, shows the MIAs reported from this genus until 2015 [6].

Conodusine A-E (370-374), apocidine A (375) and B (376), conoduzidine A (377), tabernamidine A (378) and B (379) (Figure 29) were isolated from the Malaysian stem-bark of Tabernaemontana corymbose malaysian [193]. Additionally, thirty-two compounds were also identified from the same plant, including 307, 314, 338, (+)-catharanthine (380), tabernamine (381) [194], 19'(S)-hydroxytabernamine (382) [195], and 19'(R)-hydroxytabernamine (383) [195]. 16'-decarbomethoxyvoacamine (384) [180] (Figure 29). The chemical structures were determined based on analysis of the NMR and MS spectral data. However, compounds 370, 372, 374, 375 and 377 were confirmed by X-ray diffraction analyses. 371 and 371 belong to iboga alkaloids and tabernamidine B is an iboga-containing bisindole. Tabernamidine B (379) is notable for the presence of an $\alpha$-substituted acetyl group at C-20 of the iboga carbon skeleton. The absolute configuration of (+)-conodusine E was based on an analysis of the ECD data in correlation with (-)-heyneanine and X-ray analysis. Compounds 381-384 exhibited growth inhibitory effects against drug-sensitive KB/S, with an $\mathrm{IC}_{50}$ value $<4.7 \mu \mathrm{M}$ and vincristine-resistant $(\mathrm{KB} / \mathrm{VJ} 300)$ cells with an $\mathrm{IC}_{50}$ value $<$ $4.2 \mu \mathrm{M}$. For that type of human oral cancer cell lines, vincristine was used as a reference drug with an $\mathrm{IC}_{50}$ value $<1.8 \mathrm{nM}$ [193].

Two compounds, isoakuammiline (385) and 18-hydroxypseudovincadifformine (386) [196], have been reported from the American fruits of T. litoralis. Additionally, five compounds 3,19-oxidocoronaridine (387) [196], strictosidine (388) [196], 306, heynea- 
nine 307, and tabersonine (17), have been identified from the same species [196]. Strictosidine is the major alkaloid in fruit arils, however in the capsule strictosidine it was converted to mainly iboga and pseudoaspidosperma alkaloids. However, in seeds, strictosidine was converted to both iboga and aspidosperma alkaloids, but the only major iboga alkaloid, coronaridine, was not substituted, whereas in fruit capsule coronaridine was oxidized to form heyneanine and 3,19-oxidocoronaridine.

Tabervarines A (389) and B (390) [197], 311, 369, vobasidine C (391) [198], 311, 368, ervadivaricatine B (392) [187], pedunculine (393) [199], tabernaemontanine (367) [198] and polyervine (394) [200] were published from the twigs and leaves of the Chinese T. divaricate (Figure 30). Compounds 388 and 389 exhibited a weak cytotoxic effect against cancer MCF-7, SMMC-7721, HL-60, A-549, and SW480 cells at a value > $40 \mu \mathrm{M}$ [197].

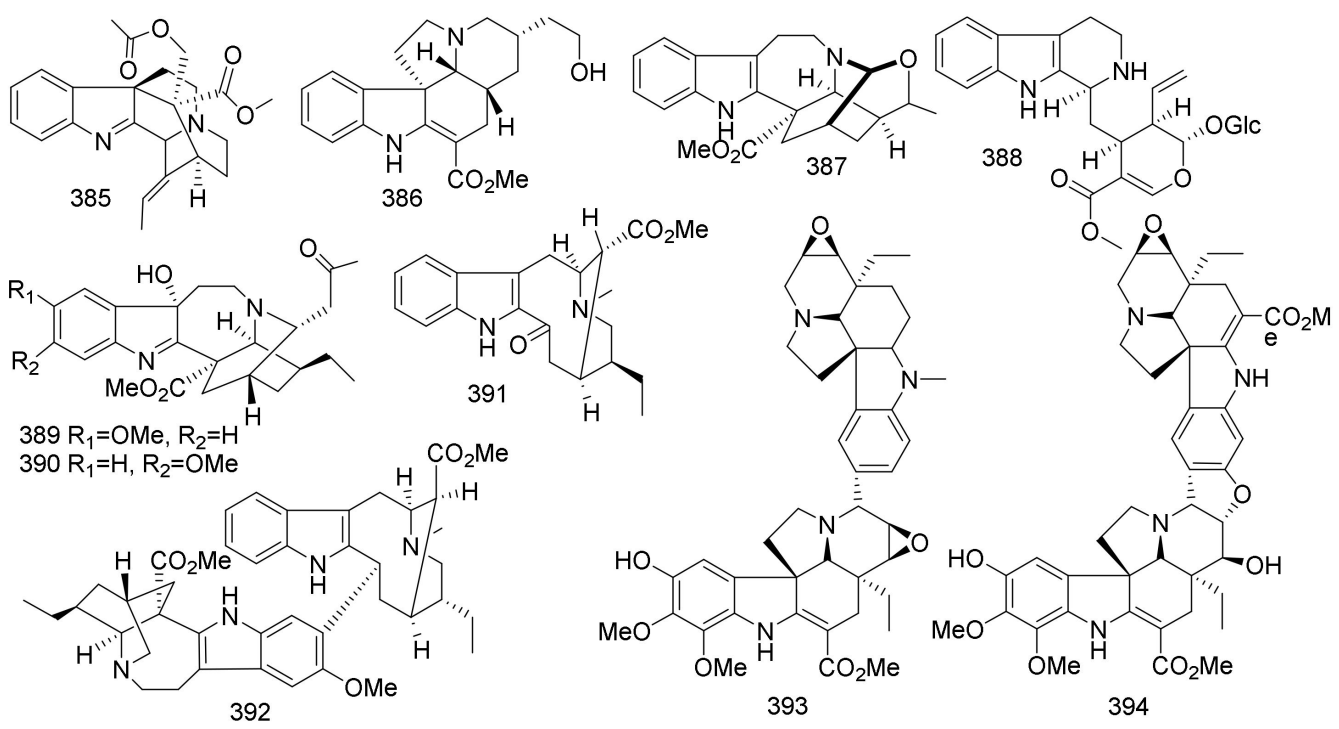

Figure 30. Compounds 385-394.

Four new bisindole compounds, flabellipparicine (395), 19,20-dihydrovobparicine (396), 10'- demethoxy-19,20-dihydrovobatensine D (397) and 3'-(2-oxopropyl)ervahanine A (398) [201], together with ten known compounds, 381, 368, ervahanine A (399) [202], vobparicine (400) [203], 19,20-dihydrotabernamine (401) [204], 19,20-dihydrotabernamine A (402) [205], taberdivarine E (403) [184], tubotaiwine (404) [206], hydroxy-3-(2-oxopropyl) coronaridineindolenine (405) [204], and deoxytubulosine (406) [201] (Figure 31) were identified from the stems of $T$. divaricate. Compounds 368, 395-403 and 406 exhibited cytotoxic activities against MCF-7 and A-549 with $\mathrm{IC}_{50}$ values $<8.1 \mu \mathrm{M}$. Compound 406 exhibited the highest effects against MCF-7 and A-549 with $\mathrm{IC}_{50}$ values of 0.1 and $0.2 \mathrm{nM}$, respectively. 7-ethyl-10-hydroxycamptothecin (SN38) was employed as a positive control and showed cytotoxic effect, with an $\mathrm{IC}_{50}$ value $<2 \mathrm{nM}$ [201]. The presence of $\beta$-carboline benzoquinolizidine nucleus played an important role in increasing the cytotoxicity in 406, whereas, compounds (368 and 395-403) possessed two NH indolic group [201].

(3R,7S,14R,19S,20R)-19-hydroxypseudovincadifformine (407) [207], 17-demethoxyhydroisorhyn chophylline (408) [208], 17-demethoxy-isorhynchophylline (409) [208], voachalotine (410) [171], 12-methoxyl-voaphylline (411) [209], and conophylline (412) [209] (Figure 32) were isolated from the branches and leaves of Chinese T. bufalina. Compound 412 showed potent cytotoxic activities against B16 and MDA-MB-231 cells with $\mathrm{IC}_{50}$ values of 0.13 and $8.9 \mu \mathrm{M}$, respectively. Gambogic acid was used as a positive control with $\mathrm{IC}_{50}$ values 22.1 and $13.5 \mu \mathrm{M}$, respectively [207]. 


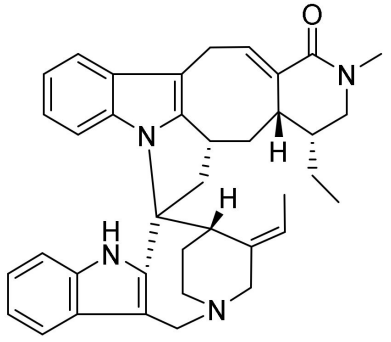

395

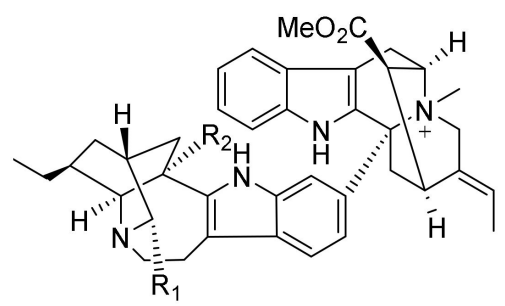

$398 \mathrm{R}_{1}=\mathrm{CH}_{2} \mathrm{COMe}, \mathrm{R}_{2}=\mathrm{CO}_{2} \mathrm{Me}$ $399 \mathrm{R}_{1}=\mathrm{H}, \mathrm{R}_{2}=\mathrm{CO}_{2} \mathrm{Me}$

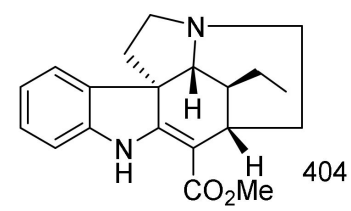

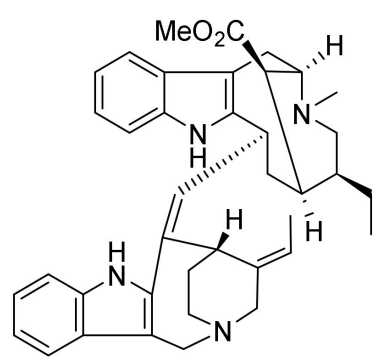
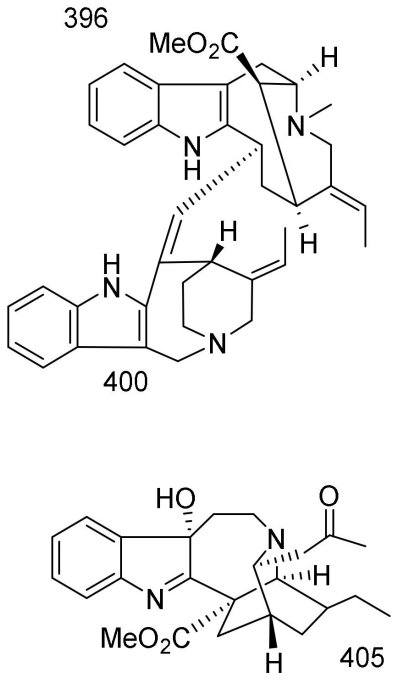

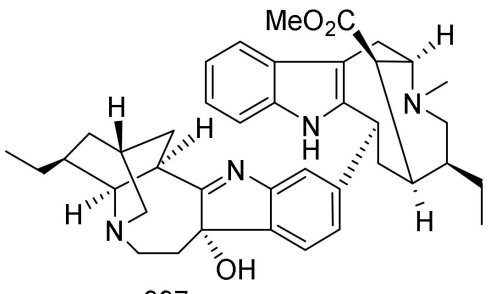

397

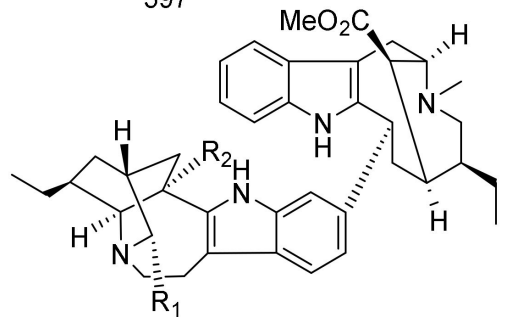

$401 \mathrm{R}_{1}=\mathrm{H}, \mathrm{R}_{2}=\mathrm{H}$

$402 \mathrm{R}_{1}=\mathrm{H}, \mathrm{R}_{2}=\mathrm{CO}_{2} \mathrm{Me}$

$403 \mathrm{R}_{1}=\mathrm{CH}_{2} \mathrm{COMe}, \mathrm{R}_{2}=\mathrm{CO}_{2} \mathrm{Me}$

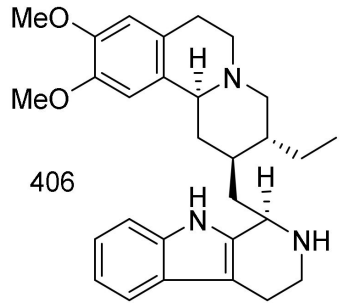

Figure 31. Compounds 395-406.

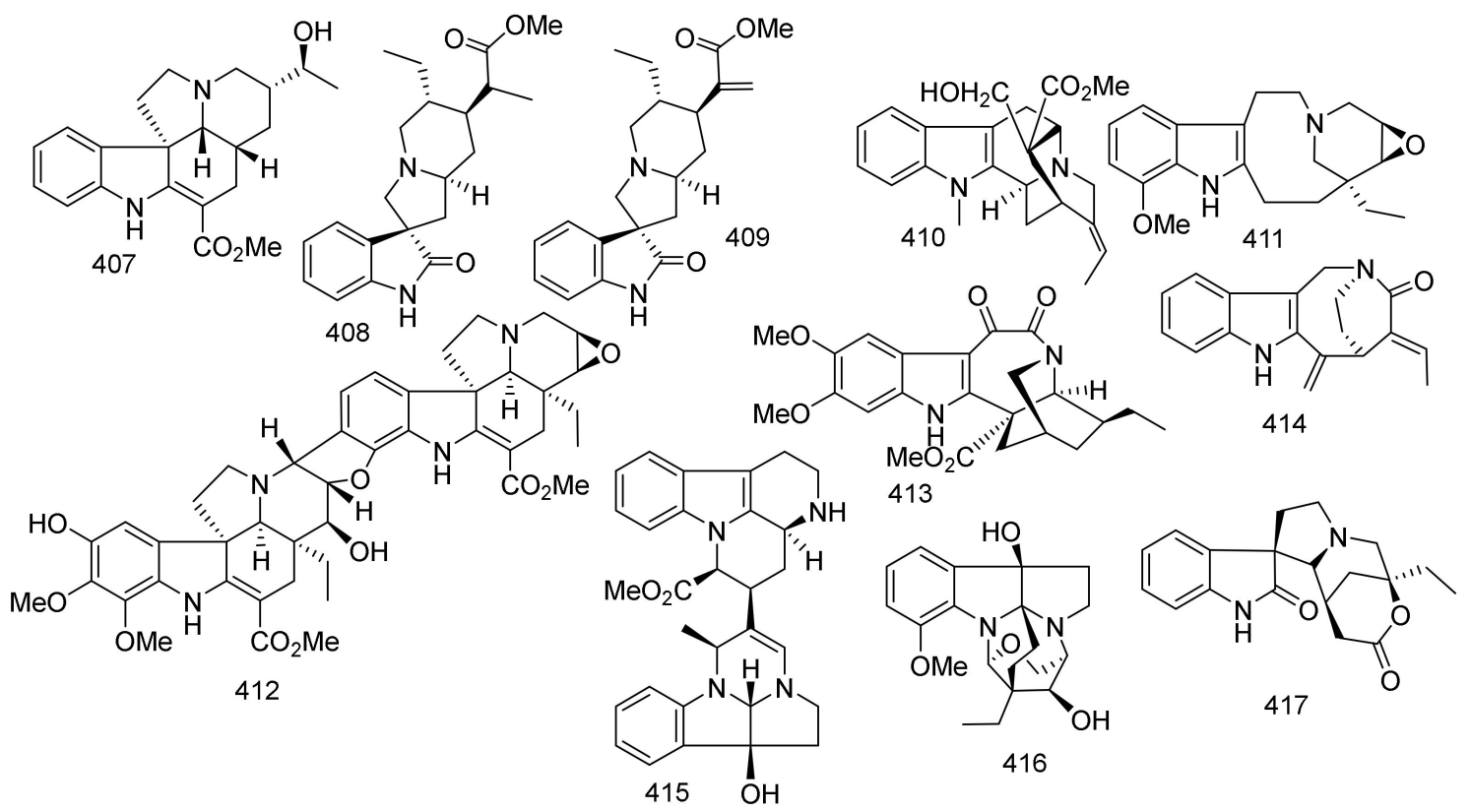

Figure 32. Compounds 407-417.

Two compounds, 5,6-dioxo-11-methoxy voacangine (413), and (-)-apparicin-21-one (414), and heyneanine (307), were identified from the fruits of cameroonean T. contorta [210] lipopolysaccharides (LPS)-stimulated RAW 264.7 macrophage cells. BAY 11-7082 was used as positive control with $10 \mu \mathrm{M}$ [210]. Tabernabovines A-C (415-417) were isolated from T. bovina [211]. Compound 415 displayed potent inhibitory activity of NO production in 
LPS-stimulated RAW 264.7 macrophages with $\mathrm{IC}_{50}$ value 44.1 value $\mu \mathrm{M}$. L-NMMA was used as a positive control and showed an inhibitory effect with $\mathrm{IC}_{50}$ value $=48.6 \mu \mathrm{M}$ [211].

Previous studies have proven that various bisindole compounds have more effect than monomeric indole compounds, including the dimeric indoles such as (euburnaneaspidospermatan, euburnane-ibogan, akuammidine-ibogan, aspidospermatan-aspidospermatan and vobasine-strychnan) type compounds. Interestingly, dimeric indoles showed more cytotoxicity than the monomeric units.

The Tabernaemontana genus produced iboga type indoles, which contained four subclasses, such as vincamine-type, apparicine-type and akuammidine, these compounds which belongs to the main class aspidosperma and corynanthe, respectively.

\section{Rhazya}

Rhazya comprises two species, Rhazya stricta (R. stricta) and Rhazya orientalis ( $R$. stricta) [212]. R. orientalis grown in western Thrace and northeastern Turkey [213] whereas, R. stricta is grown in South Asia (Afghanistan, Pakistan and India) and on the Arabian Peninsula (Saudi Arabian, Qatar, UEA, Iraq) and Iran. Rhazya is a rich source of indolecontaining compounds. Traditionally, it is has been used to cure various diseases, such as fever, rheumatism, inflammation, skin infections, sore throat, diabetes, and stomach disorders. For example, strictanol, sewarine, tetrahydrosecamine vallesiachotamine and tetrahydrosecaminediol exhibit anticancer properties [213-218]. A recent study on the aerial parts of $R$. stricta by Ahmad et al. [215], several MIAs were isolated including, three new, secopleiocarpamine A (418), 16,17-Epoxyisositsirikine (419), and 2-Ethyl-3[2-(3-ethyl1,2,3,6-tetrahydropyridine)ethyl]-indole (420) [215] (Figure 33), five previously reported compounds from other Apocynaceae genera (126, 127, 133, 298 and 404), and a number of previously isolated MIAs from the same species: 2-ethyl-3[2-(3-ethylpiperidine)ethyl]indole (421), tetrahydrosecodine (422), 16,17-dihydrosecodine (423) [216], deacetylakuammilin (424) [217], rhazimal (425), strictamine- $N$-oxide (426) [218], rhazinaline (427) [212], rhazinaline $N_{\mathrm{b}}$-oxide (428) [219], akuammicine (429) [220], 16R-E-isositsirikine (430) [221], dihydrositsirikine (431) [222], antirhine (432) [129], vincadifformine $N_{(4)}$-oxide (433) [223], eburenine (434) [93], winchinine B (435), quebrachamine (436) [224] and strictanol (437) (Figure 33) [215,225] were isolated from $R$. stricta. Furthermore, 16-epi-stemmadenine- $N$ oxide (438) (Figure 33), stemmadenine- $N$-methyl (439), and 20-epi-antirhine (440) were reported from $R$. stricta [226]. Additionally, isopicrinine (441) was isolated from the leaves of R. stricta, collected from Bahra, Saudi Arabia [227]. Abdul-Hameed et al. (2021) [228] identified two new indole alkaloids named, epirhazyaminine (442) and 20-epi-sitsirikine (443), together with five known compounds, 430, 432, 434, 437 and strictamine (444) were obtained from the aerial parts of $R$. stricta, collected from AL-Madinah city, Saudi Arabia [228]. Compounds 418, 422, 428, 432, 434, and 436 exhibited moderate growth inhibitory activities toward Candida strains (C. guilliermondii, C. albicans, C. krusei, C. lusitaniae and C. glabrata) with MIC values ranging from 3.125 to $50 \mu \mathrm{g} / \mathrm{mL}$. (Amphotericin B, MIC value < $1 \mu \mathrm{g} / \mathrm{mL}$ ) [213]. Compound 438 displayed a cytotoxic effect against HCT-116, PC-3, and HepG2, with $\mathrm{IC}_{50}$ values $=2.20,2.25$, and1.9 $\mu \mathrm{M}$, respectively, (Cisplatin, $\mathrm{IC}_{50}$ values $\leq$ $0.90 \mu \mathrm{M})$. Furthermore, compound 439 significantly hindered of the cancer cells to migration and preventing the wound healing at 24 and $48 \mathrm{~h}$ (from 81 and $77 \%$ to 68 and $46 \%$, respectively). It also inhibited proliferation and prevented cell migration of all cancer cell was evaluated, with an $\mathrm{IC}_{50}=70 \mu \mathrm{M}$ [223]. Compound 441 displayed a potent cytotoxic effect towards MCF-7, with an $\mathrm{IC}_{50}$ value $=240 \mu \mathrm{M}$ [224]. Compounds 430, 432, 434, 437, and 442-444 displayed weak activities against three cancer cell lines (HCT-116, PC-3, and HepG2), with $\mathrm{IC}_{50}$ in the range of $45.0 \pm 0.012$ and $85.0 \pm 0.068 \mu \mathrm{M}$ against HCT-116, $\mathrm{IC}_{50}$ in the range $39.0 \pm 0.012$ and $87.0 \pm 0.068 \mu \mathrm{M}$ against $\mathrm{PC}-3$, and $\mathrm{IC}_{50}$ in the range $72.0 \pm 0.164$ and $87.0 \pm 0.032 \mu \mathrm{M}$ against HepG-2 $\mu \mathrm{M})$ against HepG-2 [225]. The Rhazya genus contains many MIAs subclasses, such as secodine-type (420-424), akuammilinetype (426), akummicine-type (428) and picrinine-type (441), (Figure 3), with compounds belonging to the main classes aspidosperma and corynanthe. 


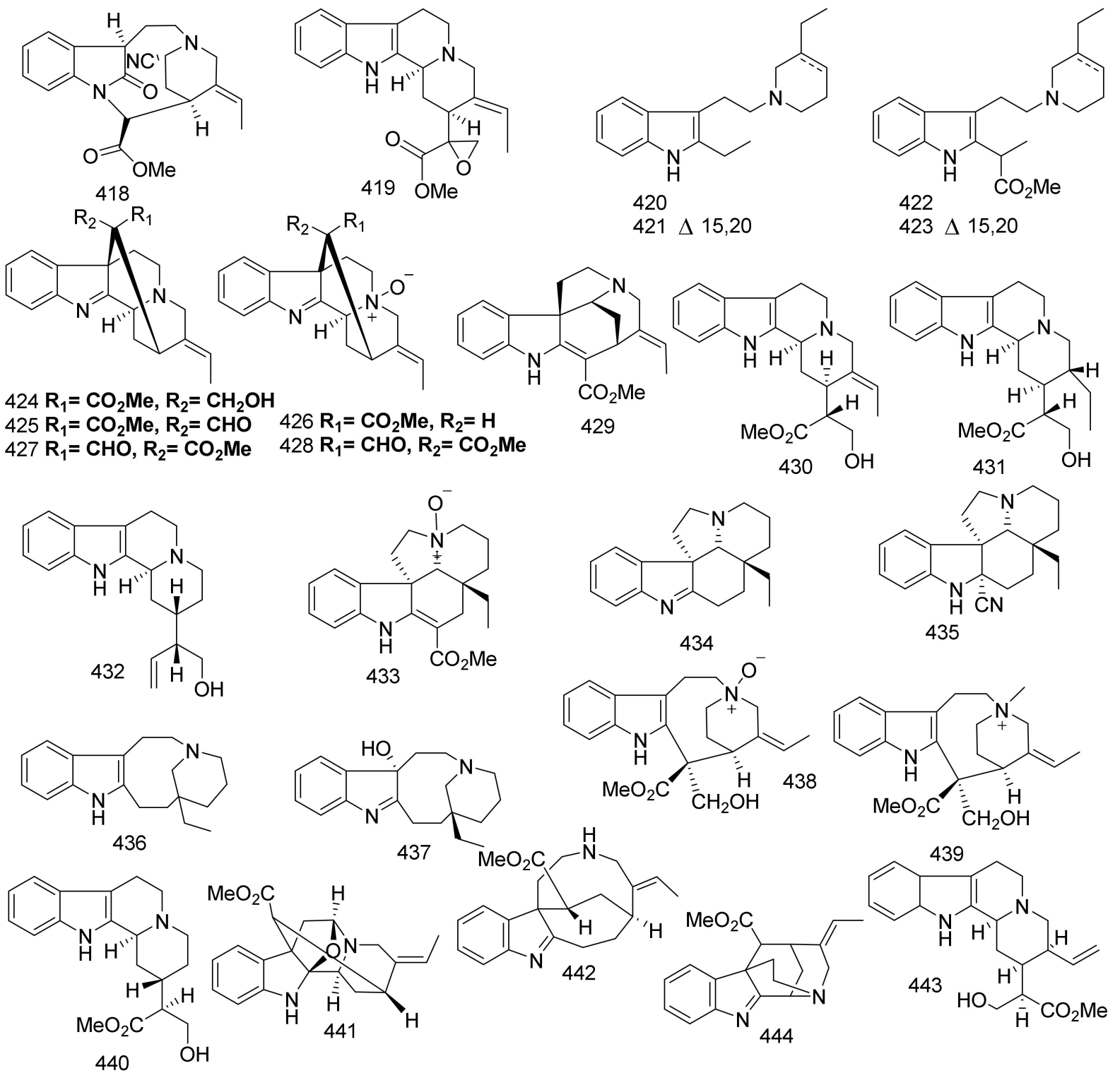

Figure 33. Compounds 418-444.

\section{Biosynthesis of Monoterpenoid Indole Alkaloids}

Monoterpenoidal indoles are obtained from the reaction of tryptamine with secologanin terpenoid. Condensation of tryptamine with Secologanin produces strictosidine by the Mannich-link reaction. The deglycosylation of strictosidine converts it to a hemiacetal. Opening the hemiacetal led to forming an aldehyde group, which then reacts with the (N-4) secondary amine of strictosidine to form 4,21-dehydrocorynanthenine. Allylic isomerization moves the double bond of vinyl to a conjugation with iminium nitrogen that generates dehydrogeissoschizine, which is then cyclized to form cathenamine. The reduction of cathenamine in the presence of NADPH forms ajmalicine (corynanthe-type) [229].

The formation of Preakuammicine occurs from dehydrogeissoschizine. Preakuammicine intermediate (strychnos-type) is the common precursor of the strychnos, aspidosperma and iboga indole alkaloids. Preakuammicine reduced to form stemmadenine, then rearranged to form the acrylic ester dehydrosecodine, which is a common intermediate for iboga and aspidosperma skeletons. Tabersonine (aspidosperma type) and catharanthine (iboga type) are formed the Diels-Alder reaction (Scheme 1) [229]. 

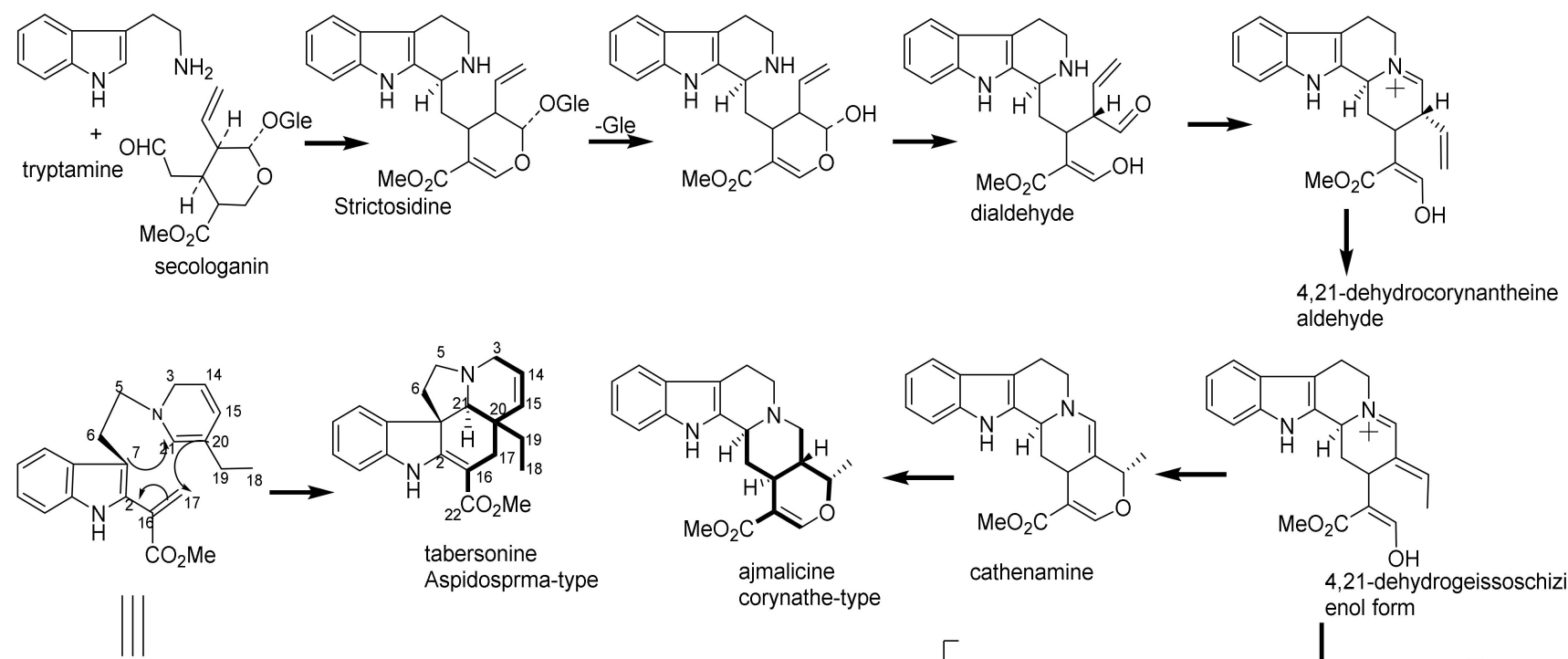

aldehyde
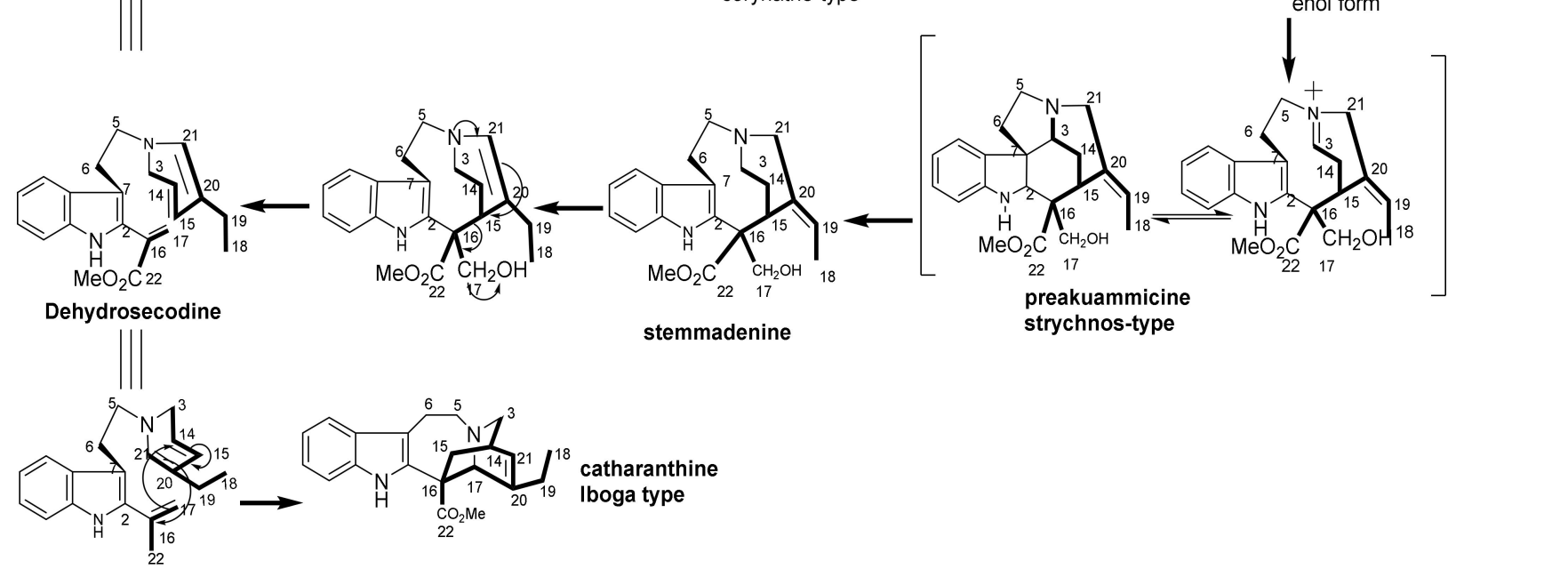

Scheme 1. Biosynthesis of corynanthe, aspidosperma and iboga indoles.

Polyneuridine aldehyde (sarpagan type) is an intermediate compound of the ajmaline pathway. The possibility of a mechanism where the sarpagan bridge enzyme converts an isomer of 4,21-dehydrogeissoschizine to polyneuridine aldehyde is shown (Scheme 2). Polyneuridine aldehyde methyl ester is hydrolyzed by polyneuridine aldehyde esterase, generating an acid which decarboxylates, to yield epi-vellosamine. Epi-vellosamine transforms to the ajmaline alkaloid vinorine. The hydroxylation of vinorine to vomilene is caused by the vinorine hydroxylase enzyme. After formation of vomilene, two step reduction occurs. First, the indolenine bond is reduced by an NADPH enzyme to yield 1,2-dihydrovomilenene. The second step, reducing the 1,2-dihydrovomilenene to acetylnorajmaline by a 1,2-dihydrovomilenene reductase enzyme. The acetyl linkage of acetylnorajmaline is hydrolyzed by acetylesterase to yield norajmaline. Finally, the production of ajmaline by $\mathrm{N}$-methyl transferase of a methyl group at the indole nitrogen of norajmaline occurs (Scheme 2) [229,230].

It is noteworthy to mention that, sarpagine, ajmaline, and macroline alkaloids are biosynthetically similar or all derived from the same origin. Whereas, sarpagine can be converted into macroline by means of Michael addition [231], on the other hand macroline can be converted into sarpagine by through a retro-Michael reaction [231-233]. Similarly, some sarpagine-containing alkaloids can be converted into ajmalines under strong acidic conditions, which refers to the great similarity between them [233]. 


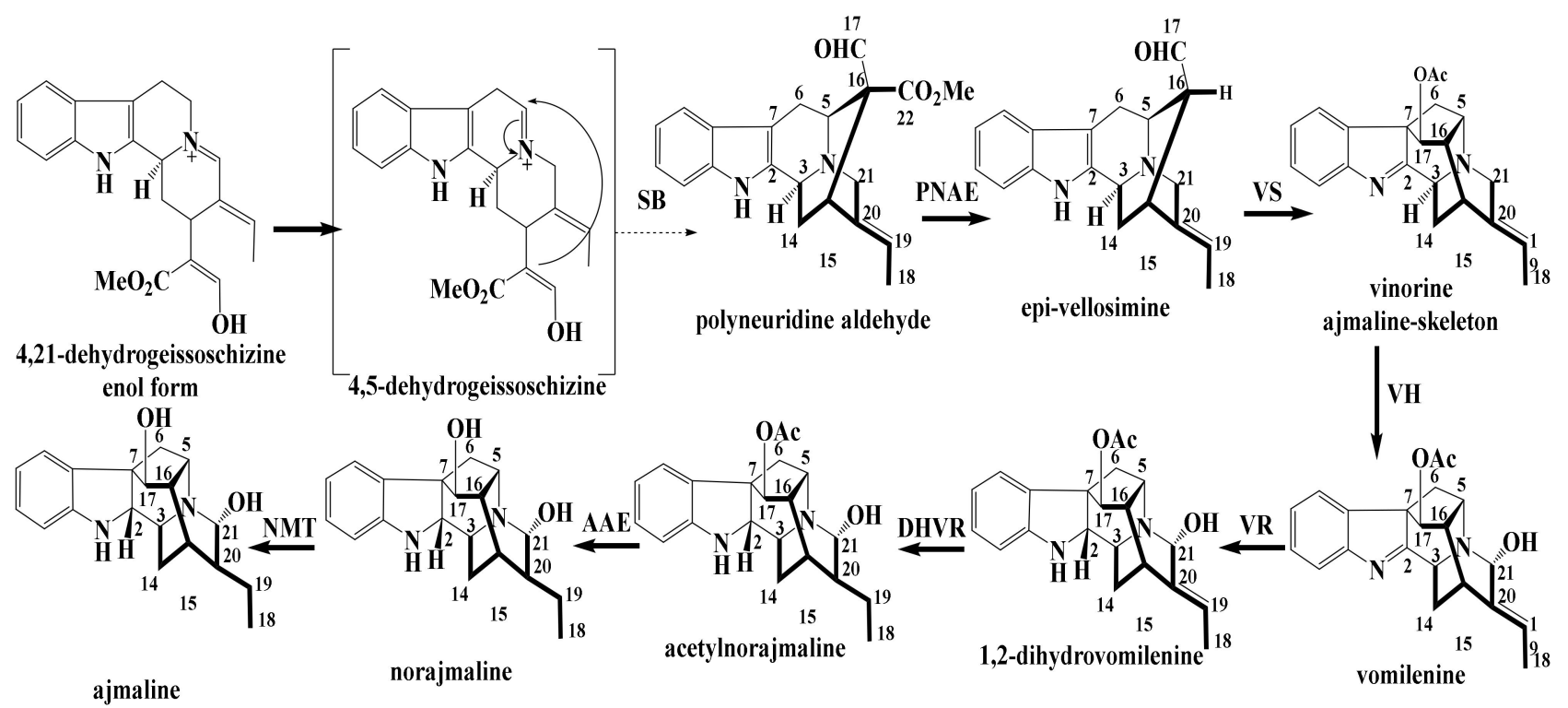

Scheme 2. Biosynthesis of ajmaline indole alkaloids. (SB) Sarpagan bridge enzyme; polyneuridine aldehyde reductase (PNAE), vinorine synthase (VS), vinorine hydroxylase (VH), vomilenine reductase (VR), dihydrovomilenine reductase (DHVR) 17-O-acetyl-ajmalanesterase (AAE), norajmaline- $N$-methyltransferase (NMT).

\section{Conclusions and Future Prospectives}

Natural products have an unprecedented molecular conformity with a diversity of functionalities. These characteristics enable them to produce biological effects, which validates the initial step for a drug lead. In recent years, the majority of new drugs reported have been natural or originated from natural sources. Alkaloids are an important source of drugs. It is noteworthy that, many alkaloids displaying fascinating molecular structures with diverse physiological and pharmacological effects have been isolated from plant families. The Apocynaceae family has been noted as a unique producer of biologically active natural metabolites such as vincristine, vinblastine, reserpine and yohimbine. This review is interested in discussing the metabolites produced from six genera belong to the family Apocynaceae. These six genera contain 400 species, which represent $20 \%$ of the Apocynaceae family. Only 30 species, which represent $7.5 \%$ of the total species of the six genera were studied. Chemical investigation of these genera led to the reporting of 444 MIAs, in the period between 2010 until December 2020, which were discussed in this review.

Figure 34 illustrates the number of compounds isolated from the six species; there are $157(35.4 \%), 126(28.4 \%), 66(14.9 \%), 48(10.8 \%), 27(6.1 \%)$, and $20(4.4 \%)$, from Alstonia, Kopsia, Ervatamia, Tabernaemontana, Rhazya and Rauvolfia, respectively. We believe that the six genera are interesting candidate for further investigation. This record coincided with the data illustrated in Figure 35. For example, Alstonia scholaris is a species that belongs to the genus Alstonia that has produced the highest number of MIAs (71 compounds) and represents $45.2 \%$ of the MITs identified from the same genus between 2010 and 2020 . The second and third most interesting species are Kopsia officinalis and Kopsia pauciflora which produced 45 and 27 compounds, respectively. These two species represent $35.7 \%$ and $21.4 \%$ of the total compounds produced from the genus Kopsia. The fourth most interesting species belong to the genus Alstonia (Alstonia mairei), which produced 26 compounds and represents $16.5 \%$ of the MITs identified from the genus Alstonia. 


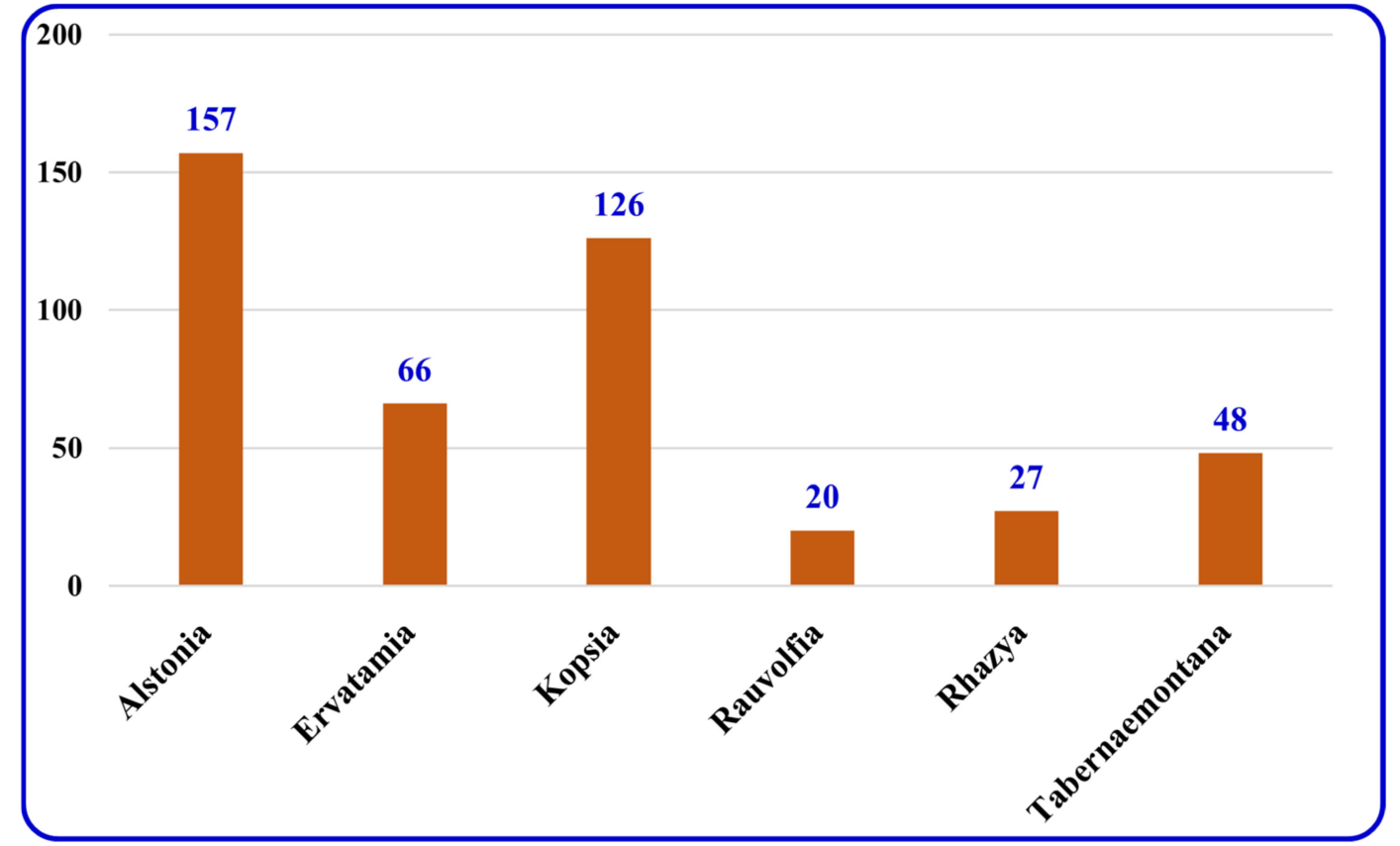

Figure 34. Number of compounds isolated from the six genera.

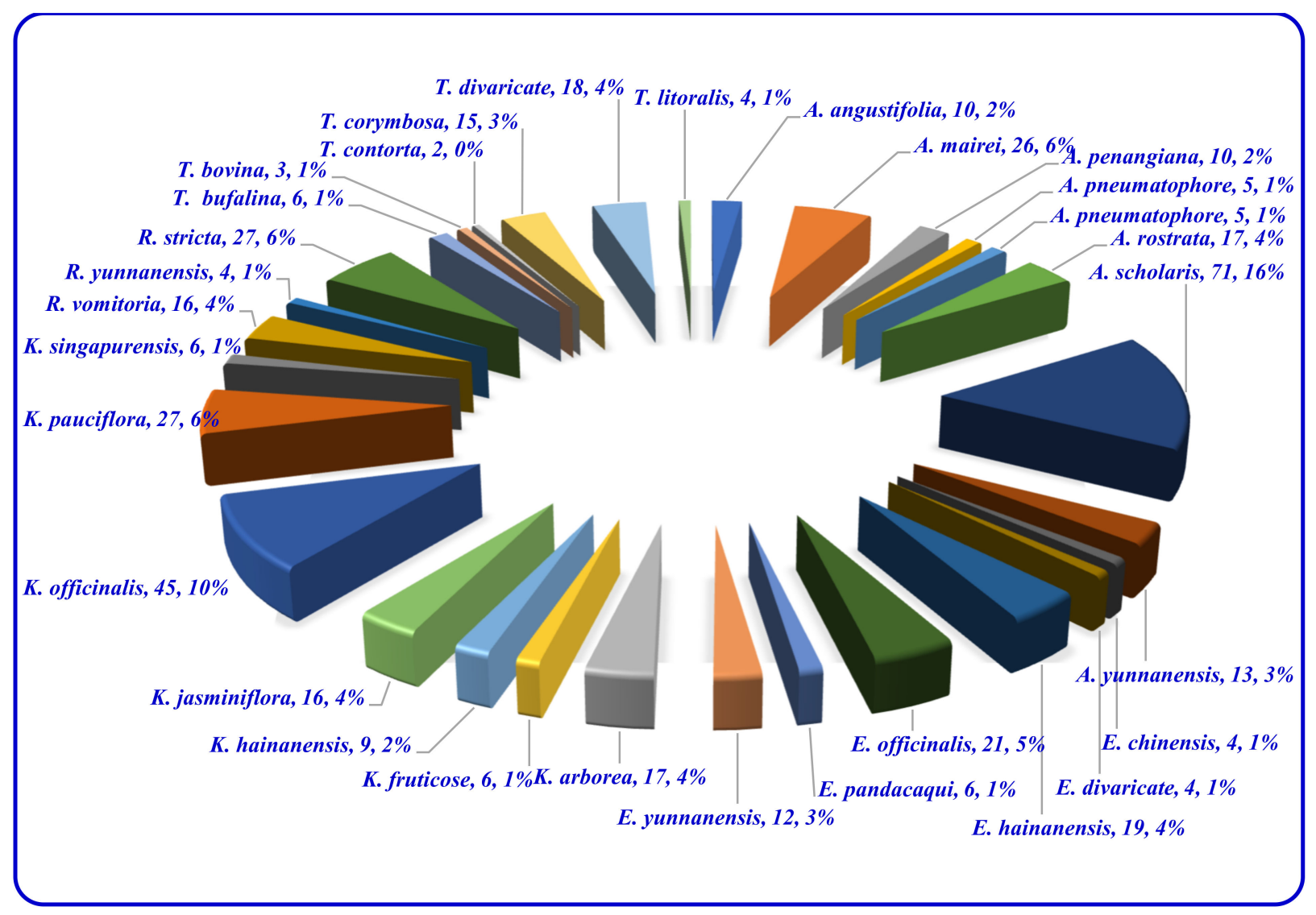

Figure 35. Percentage of reported compounds from the species.

It is interesting that the majority of compounds were isolated from twigs and leaves as illustrated in Figure 36. Additionally, the majority of the examined species belonging to the selected six genera were Chinese species and led to the identification of 360 compounds. 


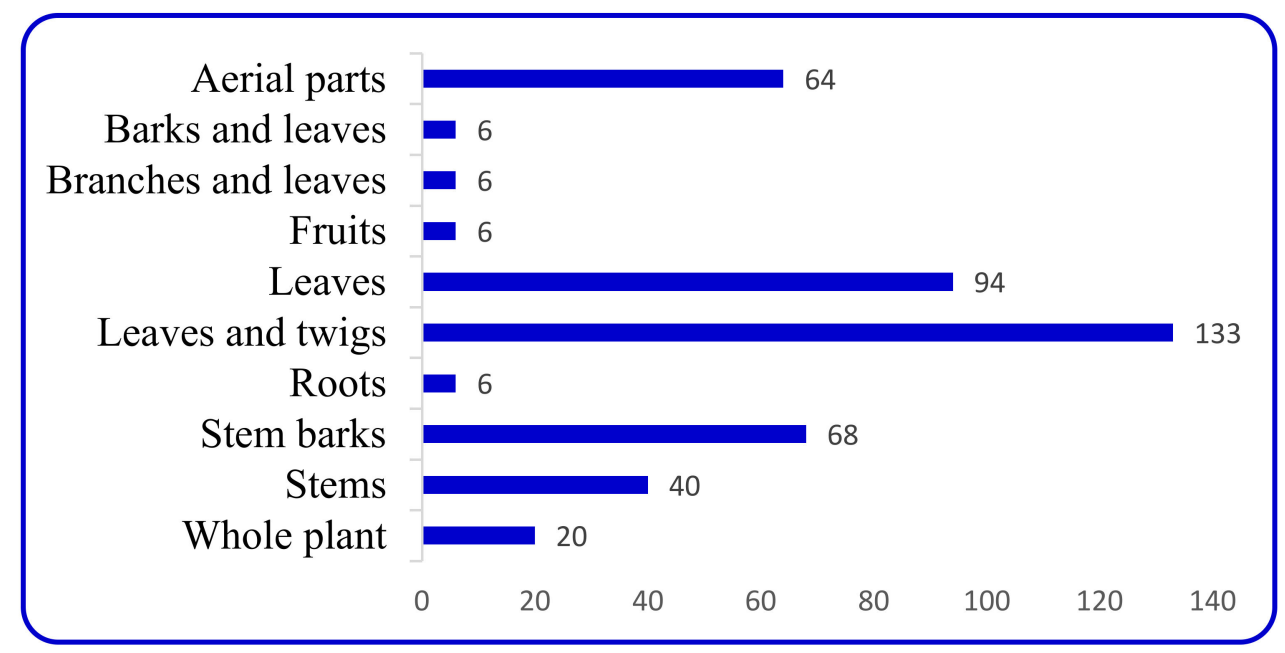

Figure 36. Number of compounds identified from different organs.

Figure 37 presents the biological activities of the compounds. The prominent activity was cytotoxicity followed by anti-inflammatory and antimicrobial activities. Thus, these compounds could be a source of anticancer drugs.

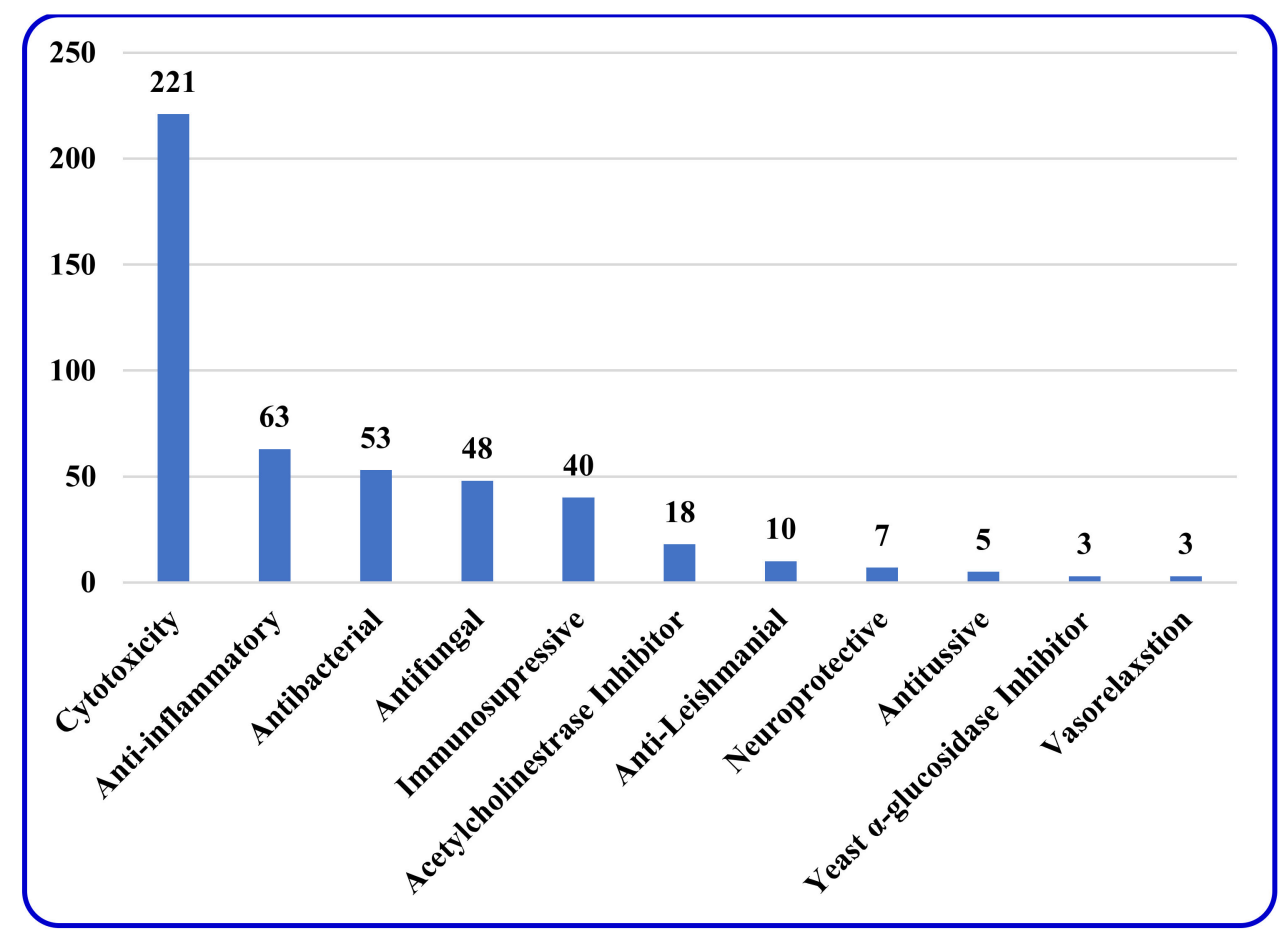

Figure 37. Number of compounds versus biological activities.

The family of terpene indole alkaloids has been discovered for over a century. There are numbers of total syntheses studies of these intricate scaffolds have been achieved. Additionally, several reviews and book chapters, as well as the references therein, are interested in the synthetic efforts have been reported.

Author Contributions: Conceptualization, W.M.A., A.A.-L. and Z.H.A.-H.; resources, A.E.M., M.O.A. and N.O.B.; data curation, Z.H.A.-H., W.M.A. and A.A.-L.; writing-original draft prepa-ration, Z.H.A.-H., W.M.A. and A.A.-L.; writing-review and editing, Z.H.A.-H., W.M.A. and A.A.-L.; supervision, T.R.S.; funding acquisition, A.E.M. and M.O.A. All authors have read and agreed to the published version of the manuscript. 
Funding: This research was funded by the Deanship of Scientific Research at Princess Nourah Bint Abdulrahman University through the Fast-track Research Funding Program.

Acknowledgments: The authors acknowledge with thanks Deanship of Scientific Research at Princess Nourah bint Abdulrahman University, for funding through the Fast-track Research Funding Program.

Conflicts of Interest: The authors declare no conflict of interest.

\begin{tabular}{|c|c|}
\hline \multicolumn{2}{|c|}{ Abbreviations } \\
\hline A431 & Dermatoma cancer \\
\hline A-549 & Lung cancer \\
\hline $\mathrm{AChE}$ & Acetylcholinesterase \\
\hline B16F10 & Melanogenesis activity \\
\hline BEN-MEN-1 & Meningioma \\
\hline BGC-823 & Human gastric carcinoma \\
\hline CAL-27 & Head and neck squamous cell carcinomas \\
\hline CCF-STTG1 & Astrocytoma \\
\hline CHG-5 & Glioma \\
\hline CI & Confidence intervals \\
\hline Detroit-562 & Head and neck squamous cell carcinomas \\
\hline $\mathrm{ED}_{50}$ & Median effective dose \\
\hline F.sp. & $\begin{array}{l}\text { Forma specialis, abbreviated f. sp., is an informal taxonomic grouping allowed by the } \\
\text { International Code of Nomenclature for algae, fungi, and plants }\end{array}$ \\
\hline HCT 116 & Human colorectal carcinoma \\
\hline $\mathrm{HeLa}$ & Human Gastric cancer \\
\hline Hep-2 & Head and neck squamous cell carcinomas \\
\hline HepG2 & Human hepatocellular \\
\hline HIF- $\alpha$ & Hypoxia-inducible factor \\
\hline HL-60 & Human myeloid leukemia \\
\hline HS-1 & Dermatona cancer \\
\hline HS-4 & Dermatona cancer \\
\hline HT-29 & Human colorectal carcinoma \\
\hline $\mathrm{IC}_{50}$ & Half maximal inhibitory concentration \\
\hline $\mathrm{ID}_{50}$ & Median infective dose \\
\hline IL-1 $\beta$ & Interleukin 1 beta \\
\hline LNCaP & Human prostate carcinoma \\
\hline M663 & Osteosarcoma cells \\
\hline MCF-7 & Human breast cancer \\
\hline MDA-MB-231 & Human breast adenocarcinoma \\
\hline MG-63 & Osteosarcoma cells \\
\hline MIAs & Terpenoid indole compounds \\
\hline MIAs & Monoterpenoid indole compounds \\
\hline MIC & Minimum inhibitory concentration \\
\hline NF-kB & Nuclear factor k-light-chain-enhancer of activated B cells \\
\hline $\mathrm{NO}$ & Nitric oxide \\
\hline PANC-1 & Pancreatic cancer \\
\hline PC-3 & Human prostate carcinoma \\
\hline PGE2 & Prostaglandin E2 \\
\hline SAOS-2 & Osteosarcoma cell lines \\
\hline SCC-PKU & Head and neck squamous cell carcinomas \\
\hline SCL-1 & Head and neck squamous cell carcinomas \\
\hline SGC-7901 & Gastric cancer \\
\hline SHG-44 & Human glioma cancer \\
\hline SK-BR-3 & Human breast cancer \\
\hline SK-MEL-2 & Human skin cancer \\
\hline SMMC-7721 & Hepatocellular carcinoma \\
\hline SOSP-9607 & Human Osteosarcoma cell lines \\
\hline SW480 & Human Colon cancer \\
\hline TCA-83 & Head and neck squamous cell carcinomas \\
\hline TNF- $\alpha$ & Tumor necrosis factor- $\alpha$ \\
\hline $\mathrm{U} 251$ & Human glioma cancer \\
\hline $\mathrm{U} 2-\mathrm{OS}$ & Osteosarcoma cell lines \\
\hline UMSCC-1 & Head and neck squamous cell carcinomas \\
\hline
\end{tabular}




\section{References}

1. Dey, A.; Mukherjee, A.; Chaudhury, M. Alkaloids from Apocynaceae: Origin, pharmacotherapeutic properties, and structureactivity studies. Stud. Nat. Prod. Chem. 2017, 52, 373-488.

2. Mondal, A.; Gandhi, A.; Fimognari, C.; Atanasov, A.G.; Bishayee, A. Alkaloids for cancer prevention and therapy: Current progress and future perspectives. Eur. J. Pharmacol. 2019, 858, 172472. [CrossRef] [PubMed]

3. Rosales, P.F.; Bordin, G.S.; Gower, A.E.; Moura, S. Indole alkaloids: 2012 until now, highlighting the new chemical structures and biological activities. Fitoterapia 2020, 143, 104558. [CrossRef] [PubMed]

4. Marcos, I.S.; Moro, R.F.; Costales, I.; Basabe, P.; David, D. Sesquiterpenyl Indoles. Nat. Prod. Rep. 2013, 30, 1509-1526. [CrossRef]

5. De Luca, V.; Salim, V.; Thamm, A.; Masada, S.A.; Yu, F. Making iridoids/secoiridoids and monoterpenoid indole alkaloids: Progress on pathway elucidation. Curr. Opin. Plant. Biol. 2014, 19, 35-42. [CrossRef]

6. Marinho, F.F.; Simões, A.O.; Barcellos, T.; Moura, S. Brazilian Tabernaemontana genus: Indole alkaloids and phytochemical activities. Fitoterapia 2016, 114, 127-137. [CrossRef]

7. Wang, C.H.; Wang, G.C.; Wang, Y.; Zhang, X.Q.; Huang, X.J.; Zhang, D.M.; Chen, M.F.; Ye, W.C. Cytotoxic dimeric indole alkaloids from Catharanthus roseus. Fitoterapia 2012, 83, 765-769. [CrossRef]

8. Hanafy, M.S.; Matter, M.A.; Asker, M.S.; Rady, M.R. Production of indole alkaloids in hairy root cultures of Catharanthus roseus L. and their antimicrobial activity. South. African J. Bot. 2016, 105, 9-18. [CrossRef]

9. Le Men, J.; Taylor, W.I. A uniform numbering system for indole alkaloids. Experientia 1965, 21, 508-510. [CrossRef]

10. Facchini, P.J. Regulation of alkaloid biosynthesis in plants. In the alkaloids. Chem. Biol. 2006, 63, 1-44.

11. El-Sayed, M.; Verpoorte, R. Catharanthus terpenoid indole alkaloids: Biosynthesis and regulation. Phytochem. Rev. 2007, 6, 277-305. [CrossRef]

12. Zhu, G.Y.; Yao, X.J.; Liu, L.; Bai, L.P.; Jiang, Z.H. Alistonitrine A, a caged monoterpene indole alkaloid from Alstonia scholaris. Org. Lett. 2014, 16, 1080-1083. [CrossRef] [PubMed]

13. Zhu, X.; Zeng, X.; Sun, C.; Chen, S. Biosynthetic pathway of terpenoid indole alkaloids in Catharanthus roseus. Front. Med. 2014, 8 , 285-293. [CrossRef] [PubMed]

14. Wong, S.K.; Lim, Y.Y.; Ling, S.K.; Wei, E.; Chan, C. Caffeoylquinic acids in leaves of selected Apocynaceae species: Their isolation and content. Pharmacognosy Res. 2014, 6, 67-72.

15. Endress, M.E.; Bruyns, P.V. A revised classification of the Apocynaceae sl. Botanical Rev. 2000, 66, 1-56. [CrossRef]

16. Góngora-Castillo, E.; Childs, K.L.; Fedewa, G.; Hamilton, J.P.; Liscombe, D.K.; Magallanes-Lundback, M.; Mandadi, K.K.; Nims, E.; Runguphan, W.; Vaillancourt, B.; et al. Development of transcriptomic resources for interrogating the biosynthesis of monoterpene indole alkaloids in medicinal plant species. PLoS ONE 2012, 7, e52506. [CrossRef]

17. Kaushik, N.K.; Kaushik, N.; Attri, P.; Kumar, N.; Kim, C.H.; Verma, A.K.; Choi, E.H. Biomedical importance of indoles. Molecules 2013, 18, 6620-6662. [CrossRef]

18. Cai, X.; Du, Z.; Luo, X. Unique monoterpenoid indole alkaloids from Alstonia scholaris. Org. Lett. 2007, 9, 1817-1820. [CrossRef]

19. Hirasawa, Y.; Arai, H.; Zaima, K.; Oktarina, R.; Rahman, A.; Ekasari, W.; Widyawaruyanti, A.; Indrayanto, G.; Zaini, N.C.; Morita, H. Alstiphyllanines A-D, indole alkaloids from Alstonia macrophylla. J. Nat. Prod. 2009, 72, 304-307. [CrossRef]

20. Adotey, J.P.K.; Adukpo, G.E.; Opoku, B.Y.; Armah, F.A. A Review of the ethnobotany and pharmacological importance of Alstonia boonei De Wild (Apocynaceae). ISRN Pharmacol. 2012, 2012, 1-9. [CrossRef]

21. Cai, X.; Zeng, C.; Feng, T.; Li, Y.; Luo, X. Monoterpenoid indole alkaloids from Alstonia mairei. Helv. Chim. Acta 2010, 93, 2037-2044. [CrossRef]

22. Ahond, A.; Janot, M.-M.; Langlois, N.; Lukacs, G.; Potier, P.; Rasoanaivo, P.; Sangare', M.; Neuss, N.; Plat, M.; Le Men, J.; et al. Carbon-13 nuclear magnetic resonance spectroscopy of naturally occurring substances. XXIII. structure of vindolinine. Am. Chem. Soc. 1974, 96, 633-634. [CrossRef]

23. Dçpke, W.; Meisel, H.; Grndemann, E. Stereochemie des 16-methoxyminovincinins. Tetrahedron Lett. 1971, 12, 1287-1290.

24. Majumder, P.L.; Basu, A. Absolute-configuration at C-19 of 19-hydroxy acyloxy-(+)-vincadifformine type of alkaloids. Indian J. Chem. Sect. B-Org. Chem. Incl. Med. Chem. 1985, 24, 649-650.

25. Majumbar, P.L.; Joardar, S.; Chanda, T.K.; Dinda, B.N.; Banerjee, M.; Ray, A.B.; Chatteriee, A. Structures and absolute sterochemistry of (-)-echitoveniline, (-)-11-methoxyechitoveniline and (-)-11-methoxyechitovenedine - new indole alkaloids of Alstonia venenata R.Br. Tetrahedron 1979, 35, 1151-1157.

26. Majumder, P.L.; Chanda, T.K.; Dinda, B.N. Structure of echitovenaldine: A new alkaloid of the leaves of Alstonia veneneta R. Br. Chem. Ind. L. 1973, 21, 1032-1033.

27. Das, B.; Biemann, K.; Chatterjee, A.; Ray, A.B.; Majumder, P. The alkaloids of the fruits of Alstonia Venenata R. Br. echitovenidine and (+)-minovincinine. Tetrahedron Lett. 1966, 22, 2483-2486. [CrossRef]

28. Majumder, P.L.; Dinda, B.N. Echitoserpidine: A new alkaloid of the fruits of Alstonia venenata. Phytochemistry 1974, 13, 645-648. [CrossRef]

29. Majumder, P.L.; Joardar, S.; Dinda, B.N.; Bandyopadhyay, D.; Basu, A. Structure and absolute stereochemistry of 19-epi-(+)echitoveniline: A new Indole alkaloid of the leaves of Alstonia venenata $\mathrm{r}$. Br. Tetrahedron 1981, 37, 1243-1248. [CrossRef]

30. Moza, B.K.; Trojanek, J.; Bose, A.K.; Das, K.G.; Funke, P. The structure of lochnericine and lochnerinine. Tetrahedron Lett. 1964, 5, 2561-2566. 
31. Ulshafer, P.R.; Bartlett, M.F.; Dorfman, L.; Gillen, M.A.; Schlittler, E.; Wenker, E. Isolation and structure of perakine. Tetrahedron Lett. 1961, 2, 363-367. [CrossRef]

32. Abe, F.; Chen, R.F.; Yamauchi, T.; Marubayashi, N.; Ueda, I. Alschomine and isoalschomine, new alkaloids from the leaves of Alstonia scholaris. Chem. Pharm. Bull. 1989, 37, 887-890. [CrossRef]

33. Chen, W.; Yan, Y.P.; Wang, Y.J.; Liang, X.T. Isolation and identification of three new alkaloids from roots of Alstonia yunnanensis. Acta Pharm. Sin. (Yaохиехиebao) 1985, 20, 906-912.

34. Evans, D.A.; Joule, J.A.; Smith, G.F. The alkaloids of Rhazya orientalis. Phytochemistry 1968, 7, 1429-1431. [CrossRef]

35. Ahmad, Y.; Fatima, K.; Le Quesne, P.W.; Atta-ur-Rahman. Further alkaloidal constituents of the leaves of Rhazya stricta. Phyrochemistry 1983, 22, 1017-1019. [CrossRef]

36. Koyama, K.; Hirasawa, Y.; Nugroho, A.E.; Hosoya, T.; Hoe, T.C.; Chan, K.; Morita, H. Alsmaphorazines A and B, indole alkaloids from Alstonia pneumatophora. Org. Lett. 2010, 12, 4188-4191. [CrossRef]

37. Cai, X.; Bao, M.; Zhang, Y.; Zeng, C.; Liu, Y.; Luo, X. A new type of monoterpenoid indole alkaloid precursor from Alstonia rostrata. Org. Lett. 2011, 13, 3568-3571. [CrossRef]

38. Bao, M.; Zeng, C.; Qu, Y.; Kong, L.; Liu, Y.; Cai, X.; Luo, X. Monoterpenoid indole alkaloids from Alstonia rostrata. Nat. Prod. Bioprospect. 2012, 2, 121-125. [CrossRef]

39. Cao, P.; Liang, Y.; Gao, X.; Li, X.; Song, Z.; Liang, G. Monoterpenoid indole alkaloids from Alstonia yunnanensis and their cytotoxic and anti-inflammatory activities. Molecules 2012, 17, 13631-13641. [CrossRef]

40. Koyama, K.; Hirasawa, Y.; Nugroho, A.E.; Kaneda, T.; Hoe, T.C.; Chan, K.-L.; Morita, H. Alsmaphorazines C-E, indole alkaloids from Alstonia pneumatophora. Teonhedron 2012, 68, 1502-1506. [CrossRef]

41. Wang, W.; Cheng, M.H.; Wang, X.H. Monoterpenoid indole alkaloids from Alstonia rupestris with cytotoxic, anti-inflammatory and antifungal activities. Molecules 2013, 18, 7309-7322. [CrossRef] [PubMed]

42. Feng, T.; Cai, X.H.; Zhao, P.J.; Du, Z.Z.; Li, W.Q.; Luo, X.D. Monoterpenoid indole alkaloids from the bark of Alstonia scholaris. Planta Med. 2009, 75, 1537-1541. [PubMed]

43. Yang, X.; Qin, X.; Zhao, Y.; Lunga, P.K.; Li, X.; Jiang, S.; Cheng, G.; Liu, Y.; Luo, X. Alstolactines A - C, novel monoterpenoid indole alkaloids from Alstonia scholaris. Tetrahedron Lett. 2014, 55, 4593-4596. [CrossRef]

44. Zhang, L.; Hua, Z.; Song, Y.; Feng, C. Monoterpenoid indole alkaloids from Alstonia rupestris with cytotoxic, antibacterial and antifungal activities. Fitoterapia 2014, 97, 142-147. [CrossRef]

45. Pan, L.; Terrazas, C.; Acuña, U.M.; Ninh, T.N.; Chai, H.; Carcache De Blanco, E.J.; Soejarto, D.D.; Satoskar, A.R.; Kinghorn, A.D. Bioactive indole alkaloids isolated from Alstonia angustifolia. Phytochem. Lett. 2015, 10. liv-lix. [CrossRef]

46. Kam, T.S.; Choo, Y.M.; Komiyama, K. Unusual spirocyclic macroline alkaloids, nitrogenous derivatives, and a cytotoxic bisindole from Alstonia. Tetrahedron 2004, 60, 3957-3966. [CrossRef]

47. Kam, T.S.; Iek, I.H.; Choo, Y.M. Alkaloids from the stem-bark of Alstonia macrophylla. Phytochemistry 1999, 51, 839-844. [CrossRef]

48. Bi, Y.; Zhang, L.H.; Hamaker, L.K.; Cook, J.M. Enantiospecific synthesis of (-)-alstonerine and (+)-macroline as well as a partial synthesis of (+)-villalstonine. J. Am. Chem. Soc. 1994, 116, 9027-9041. [CrossRef]

49. Zocoler, M.A.; De Oliveira, A.J.B.; Sarragiotto, M.H.; Grzesiuk, V.L.; Vidotti, G.J. Qualitative Determination of indole alkaloids of Tabernaemontana fuchsiaefolia (Apocynaceae). J. Braz. Chem. Soc. 2005, 16, 1372-1377. [CrossRef]

50. Ghedira, K.; Zeches-Hanrot, M.; Richard, B.; Massiot, G.; Le Men-Olivier, L.; Sevenet, T.; Goh, S.H. Alkaloids of Alstonia angustifolia . Phytochemistry 1988, 27, 3955-3962. [CrossRef]

51. Tan, S.J.; Lim, K.H.; Subramaniam, G.; Kam, T.S. Macroline-sarpagine and macroline-pleiocarpamine bisindole alkaloids from Alstonia angustifolia. Phytochemistry 2013, 85, 194-202. [CrossRef] [PubMed]

52. Liu, L.; Chen, Y.; Qin, X.; Wang, B.; Jin, Q.; Liu, Y.; Luo, X. Antibacterial monoterpenoid indole alkaloids from Alstonia scholaris cultivated in temperate zone. Fitoterapia 2015, 105, 160-164. [CrossRef] [PubMed]

53. Qin, X.; Zhao, Y.; Lunga, P.; Yang, X.; Song, C.; Cheng, G.; Liu, L.; Chen, Y.; Liu, Y.-P.; Luo, X. Indole alkaloids with antibacterial activity from aqueous fraction of Alstonia scholaris. Tetrahedron 2015, 71, 4372-4378. [CrossRef]

54. Qin, X.J.; Zhao, Y.L.; Song, C.W.; Wang, B.; Chen, Y.Y.; Liu, L.; Li, Q.; Li, D.; Liu, Y.P.; Luo, X.D. Monoterpenoid indole alkaloids from inadequately dried leaves of Alstonia scholaris. Nat. Products Bioprospect. 2015, 5, 185-193. [CrossRef]

55. Yang, X.W.; Luo, X.D.; Lunga, P.K.; Zhao, Y.L.; Qin, X.J.; Chen, Y.Y.; Liu, L.; Li, X.N.; Liu, Y.P. Scholarisines H-O, novel indole alkaloid derivatives from long-term stored Alstonia scholaris. Tetrahedron 2015, 71, 3694-3698. [CrossRef]

56. Kuok, C.; Zhang, J.; Fan, C.; Zhang, Q.; Fan, R.; Zhang, D.; Zhang, X.; Ye, W. Meloslines A and B, Two novel indole alkaloids from Alstonia scholaris. Tetrahedron Lett. 2017, 58, 2740-2742. [CrossRef]

57. Edwards, P.N.; Smith, G.F. 287. Akuamma alkaloids. Part I V.The decomposition of akuammicine in methanol. J. Chem. Soc. 1961, 152, 1458-1462. [CrossRef]

58. Li, C.; Chen, S.; Sun, C.; Zhang, L.; Shi, X.; Wu, S. Cytotoxic monoterpenoid indole alkaloids from Alstonia yunnanensis Diels. Fitoterapia 2017, 117, 79-83. [CrossRef]

59. Yan, T.; Han, D.; Hu, J.; Huang, X.; Wang, H. Monoterpenoid indole alkaloids from Alstonia mairei and their cytotoxicity. J. Asian Nat. Prod. Res. 2017, 6020, 1-7. [CrossRef]

60. Koyama, K.; Hirasawa, Y.; Zaima, K.; Hoe, T.C.; Chan, K.L.; Morita, H. Alstilobanines A-E, new indole alkaloids from Alstonia angustiloba. Bioorganic Med. Chem. 2008, 16, 6483-6488. [CrossRef] 
61. Zhong, X.H.; Bao, M.F.; Zeng, C.X.; Zhang, B.J.; Wu, J.; Zhang, Y.; Cai, X.H. Polycyclic monoterpenoid indole alkaloids from Alstonia rostrata and their reticulate derivation. Phytochem. Lett. 2017, 20,77-83. [CrossRef]

62. Cai, X.H.; Tan, Q.G.; Liu, Y.P.; Feng, T.; Du, Z.Z.; Li, W.Q.; Luo, X.D. A cage-monoterpene indole alkaloid from Alstonia scholaris. Org. Lett. 2008, 10, 577-580. [CrossRef] [PubMed]

63. Yu, H.F.; Qin, X.J.; Ding, C.F.; Wei, X.; Yang, J.; Luo, J.R.; Liu, L.; Khan, A.; Zhang, L.C.; Xia, C.F.; et al. Nepenthe-like indole alkaloids with antimicrobial activity from Ervatamia chinensis. Org. Lett. 2018, 20, 4116-4120. [CrossRef] [PubMed]

64. Lounasmaa, M.; Jokela, R.; Hanhinen, P.; Miettenen, J.; Salo, J. The rhazimanine-bhimberine enigma. J. Nat. Prod. 1995, 58, 131-133. [CrossRef]

65. Morita, Y.; Hesse, M.; Schmid, H.; Banerji, A.; Banerji, J.; Chatterjee, A.; Oberhänsli, W.E. Alstonia scholaris: Struktur des indolalkaloides Narelin. Helv. Chim. Acta 1977, 60, 1419-1434. [CrossRef]

66. Goh, S.H.; Ali, A.R.M.; Wong, W.H. Alkaloids of Leuconotis griffithii and L. eugenifolia (Apocynaceae). Tetrahedron 1989, 45, 7899-7920. [CrossRef]

67. Hu, W.L.; Zhu, J.P.; Hesse, M. Indole alkaloids from Aistonia angustjfolia. Planta Med. 1989, 55, 463-466. [CrossRef]

68. Yamauchi, T.; Abe, F.; fu Chen, R.; Nonaka, G.I.; Santisuk, T.; Padolina, W.G. Alkaloids from the leaves of Alstonia scholaris in Taiwan, Thailand, Indonesia and the Philippines. Phytochemistry 1990, 29, 3547-3552. [CrossRef]

69. Lounasmaa, M.; Jokela, R.; Tolvanen, A.; Kan, S.K. A 400 MHz 1H-NMR study of seven sarpagine-type alkaloids. Planta Med. 1985, 6, 519-521. [CrossRef]

70. Zhang, L.; Zhang, C.J.; Zhang, D.B.; Wen, J.; Zhao, X.W.; Li, Y.; Gao, K. An Unusual indole alkaloid with anti-adenovirus and anti-HSV activities from Alstonia scholaris. Tetrahedron Lett. 2014, 55, 1815-1817.

71. Morita, H.; Ichihara, Y.; Takeya, K.; Watanabe, K.; Itokawa, H.; Motidome, M. A New indole alkaloid glycoside from the leaves of Palicourea marcgravii. Planta Med. 1989, 55, 288-289. [CrossRef] [PubMed]

72. Yang, J.; Fu, J.; Liu, X.; Jiang, Z.; Zhu, G. Monoterpenoid indole alkaloids from the leaves of Alstonia scholaris and their NF- $\mathrm{k} B$ inhibitory activity. Fitoterapia 2018, 124, 73-79. [CrossRef] [PubMed]

73. Yeap, J.S.Y.; Navanesan, S.; Sim, K.S.; Yong, K.T.; Gurusamy, S.; Lim, S.H.; Low, Y.Y.; Kam, T.S. Ajmaline, oxindole, and cytotoxic macroline-akuammiline bisindole alkaloids from Alstonia penangiana. J. Nat. Prod. 2018, 81, 1266-1277. [CrossRef] [PubMed]

74. Yuan, Y.X.; Guo, F.; He, H.P.; Zhang, Y.; Hao, X.J. Two new monoterpenoid indole alkaloids from Alstonia rostrata. Nat. Prod. Res. 2018, 32, 844-848. [CrossRef] [PubMed]

75. Ming, C.W.; Ling, Z.P.; Ruecker, G. Nb-demethylechitamine N-oxide from roots of Winchia calophylla. Planta Med. 1988, 54, 480-481. [CrossRef] [PubMed]

76. Keawpradub, N.; Takayama, H.; Aimi, N.; Sakai, S.I. Indole alkaloids from Alstonia glaucescens. Phytochemistry 1994, 37, 1745-1749. [CrossRef]

77. Gan, L.-S.; Yang, S.-P.; Wu, Y.; Jian, D.; Yue, J.-M. Terpenoid indole alkaloids from Winchia calophylla. J. Nat. Prod. 2006, 69, 18-22. [CrossRef]

78. Hu, J.; Mao, X.; Shi, X.; Jin, N.; Shi, J. Monoterpenoid indole alkaloids from the leaves of Alstonia scholaris. Chem. Nat. Compd. 2018, 54, 934-937. [CrossRef]

79. Krishnan, P.; Lee, F.K.; Chong, K.W.; Mai, C.W.; Muhamad, A.; Lim, S.H.; Low, Y.Y.; Ting, K.N.; Lim, K.H. Alstoscholactine and alstolaxepine, monoterpenoid indole alkaloids with $\gamma$-lactone-bridged cycloheptane and oxepane moieties from Alstonia scholaris. Org. Lett. 2018, 20, 8014-8018. [CrossRef]

80. Krishnan, P.; Mai, C.; Yong, K.; Low, Y.; Lim, K. Alstobrogaline, an Unusual pentacyclic monoterpenoid indole alkaloid with aldimine and aldimine- N -oxide moieties from Alstonia scholaris. Tetrahedron Lett. 2019, 60, 789-791. [CrossRef]

81. Macabeo, A.P.G.; Krohn, K.; Gehle, D.; Read, R.W.; Brophy, J.J.; Cordell, G.A.; Franzblau, S.G.; Aguinaldo, A.M. Indole alkaloids from the leaves of Philippine Alstonia scholaris. Phytochemistry 2005, 66, 1158-1162. [CrossRef] [PubMed]

82. Khyade, M.S.; Kasote, D.M.; Vaikos, N.P. Alstonia scholaris (L.) R. Br. and Alstonia macrophylla Wall. Ex G. Don: A comparative review on traditional uses, phytochemistry and pharmacology. J. Ethnopharmacol. 2014, 153, 1-18. [CrossRef] [PubMed]

83. Mason, J.D.; Weinreb, S.M. The Alstoscholarisine alkaloids: Isolation, structure determination , biogenesis , biological evaluation , and synthesis. In The Alkaloids; Elsevier Inc.: Amsterdam, The Netherlands, 2019; Volume 81, pp. 115-150.

84. Maurya, A.; Verma, S.C.; Meena, R.A.J.; Srivastava, A.; Shankar, M.B.; Sharma, R.K. Phytochemistry and chromatographic analysis of Alstonia scholaris (L.) R.Br. used as a traditional medicine: A review. In World J. Pharm. Res.; 2016; Volume 5, pp. $1503-1519$.

85. Wong, S.P.; Gan, C.Y.; Lim, K.H.; Ting, K.N.; Low, Y.Y.; Kam, T.S. Arboridinine, a pentacyclic indole alkaloid with a new cage carbon-nitrogen skeleton derived from a pericine precursor. Org. Lett. 2015, 17, 3628-3631. [CrossRef] [PubMed]

86. Cheenprachaa, S.; Raksata, A.; Ritthiwigromb, T.A.; Laphookhieoc, S. Monoterpene indole alkaloids from the twigs of Kopsia arborea. Nat. Prod. Commun. 2014, 9, 1441-1443. [CrossRef]

87. Kam, T.S.; Sim, K.M.; Koyano, T.; Komiyama, K. Leishmanicidal alkaloids from Kopsia griffithii. Phytochemistry 1999, 50, 75-79. [CrossRef]

88. Lim, K.H.; Hiraku, O.; Komiyama, K.; Koyano, T.; Hayashi, M.; Kam, T.S. Biologically active indole alkaloids from Kopsia arborea. J. Nat. Prod. 2007, 70, 1302-1307. [CrossRef]

89. Tan, M.J.; Yin, C.; Tang, C.P.; Ke, C.Q.; Lin, G.; Ye, Y. Antitussive indole alkaloids from Kopsia hainanensis. Planta Med. 2011, 77, 939-944. [CrossRef] 
90. Gan, C.; Yoganathan, K.; Sim, K.; Low, Y.; Lim, S.; Kam, T. Corynanthean, eburnan, secoleuconoxine, and pauciflorine alkaloids from Kopsia pauciflora. Phytochemistry 2014, 108, 234-242. [CrossRef]

91. Yap, W.S.; Gan, C.Y.; Sim, K.S.; Lim, S.H.; Low, Y.Y.; Kam, T.S. Aspidofractinine and eburnane alkaloids from a north Borneo Kopsia. ring-contracted, additional ring-fused, and paucidactine-type aspidofractinine alkaloids from K. pauciflora. J. Nat. Prod. 2015, 79, 230-239. [CrossRef]

92. Yap, W.S.; Gan, C.Y.; Low, Y.Y.; Choo, Y.M.; Etoh, T.; Hayashi, M.; Komiyama, K.; Kam, T.S. Grandilodines A - C, biologically active indole alkaloids from Kopsia grandifolia. J. Nat. Prod. 2011, 74, 1309-1312. [CrossRef]

93. Wu, Y.; Kitajima, M.; Kogure, N.; Wang, Y.; Zhang, R.; Takayama, H. Two New Aspidosperma Indole Alkaloids from Yunnan Kopsia Arborea. Chem. Pharm. Bull. 2010, 58, 961-963.

94. Kam, T.; Tan, P.; Hoong, P.Y.; Cheng-Hock, C. Methyl Chanofruticosinates from leaves of Kopsia arborea. Phytochemistry 1993, 32, 489-491.

95. Ahmad, K.; Hirasawa, Y.; Nugroho, A.E.; Hadi, A.H.A.; Takeya, K.; Thomas, N.F.; Awang, K.; Morita, H.; Ping, T.S.; Nafiah, M.A. New indole alkaloids from Kopsia singapurensis (RIDL.). Open Conf. Proc. J. 2013, 4, 75-82. [CrossRef]

96. Chen, J.; Yang, M.; Zeng, J.; Gao, K. New broad-spectrum antibacterial and antifungal alkaloids from Kopsia hainanensis. Phytochem. Lett. 2014, 7, 156-160. [CrossRef]

97. Kitajima, M.; Anbe, M.; Kogure, N.; Wongseripipatana, S.; Takayama, H. Indole alkaloids from Kopsia jasminiflora. Tetrahedron 2014, 70, 9099-9106. [CrossRef]

98. Feng, X.; Kan, C.; Potier, P.; Kan, S.; Lounasmaa, M. Monomeric indole alkaloids from Kopsia officinalis. Planta Med. 1983, 48, 280-282. [CrossRef]

99. Lim, K.H.; Low, Y.Y.; Tan, G.H.; Kam, T.S.; Lim, T.-M. Kopsine and danuphylline alkaloids from Kopsia. biomimetic partial synthesis of danuphylline B. Helv. Chim. Acta 2008, 91, 1559-1566. [CrossRef]

100. Trigo-Mouriño, P.; Sifuentes, R.; Navarro-Vázquez, A.; Gayathri, C.; Maruenda, H.; Gil, R.R. Determination of the absolute configuration of 19-OH-(-)-eburnamonine using a combination of residual dipolar couplings, DFT chemical shift predictions, and chiroptics. Nat. Prod. Commun. 2012, 7, 735-738. [CrossRef]

101. Feng, T.; Cai, X.H.; Liu, Y.P.; Li, Y.; Wang, Y.Y.; Luo, X.D. Melodinines A-G, Monoterpenoid indole alkaloids from Melodinus henryi. J. Nat. Prod. 2010, 73, 22-26. [CrossRef]

102. Kam, T.S.; Subramaniam, G.; Chen, W. Alkaloids from Kopsia dasyrachis. Phytochemistry 1999, 51, 159-169. [CrossRef]

103. Smith, G.F.; Wahid, M.A. 760. The Isolation of $( \pm)$ - and (+)-vincadifformine and of (+)- 1,2-dehydroaspidospermidine from Rhazya stricta. J. Chem. Soc. 1963, 4002-4004. [CrossRef]

104. Kitajima, M.; Koyama, T.; Wu, Y.; Kogure, N.; Zhang, R.; Takayam, H. Kopsiyunnanines J1 and J2, new strychnos-type Homomonoterpenoid indole alkaloids from Kopsia arborea. Nat. Prod. Commun. 2015, 10, 49-51. [CrossRef]

105. Kam, T.-S.; Arasu, L.; Yoganathan, K. Alkaloids from Koposia pauciflora. Phytochemistry 1996, 43, 1385-1387. [CrossRef]

106. Atta-ur-Rahman; Zaman, K.; Perveen, S.; Muzaffar, A.; Choudhary, M.I.; Pervin, A. Alkaloids from Rhazya Stricta. Phytochemistry 1991, 30, 1285-1293. [CrossRef]

107. Kan-Fan, C.; Sevenet, T.; Husson, H.P. Kopsia pauciflora, I. structure et configuration absolue de nouveaux alcaloïdes indoliques dimeres de type aspidospermane-eburnane de La serie pleiomutine-kopsoffine. J. Nat. Prod. 1985, 48, 124-127. [CrossRef]

108. Kitajima, M.; Nakazawa, M.; Wu, Y.; Kogure, N.; Zhang, R.P.; Takayama, H. Kopsiyunnanines L and M, strychnos-related monoterpenoid indole alkaloids from Yunnan Kopsia arborea. Tetrahedron 2016, 72, 6692-6696. [CrossRef]

109. Wong, S.; Chong, K.; Lim, K.; Lim, S.; Low, Y.; Kam, T. Arborisidine and arbornamine, two monoterpenoid indole alkaloids with new polycyclic carbon - nitrogen skeletons derived from a common pericine precursor. Org. Lett. 2016, 18, 1618-1621. [CrossRef]

110. Zeng, T.; Wu, X.; Yang, S.; Lai, W.; Shi, S.; Zou, Q.; Liu, Y.; Li, L. Monoterpenoid indole alkaloids from Kopsia officinalis and the immunosuppressive activity of rhazinilam. Nat. Prod. 2017, 80, 864-871. [CrossRef] [PubMed]

111. Klyne, W.; Swan, R.J.; Gorman, A.A.; Guggisberg, A.; Schmid, H. Optische rotationsdispersion von indolinalkaloiden mit ketogruppen. Helv. Chim. Acta 1968, 51, 1168-1184. [CrossRef]

112. Husain, K.; Jantan, I.; Kamaruddin, N.; Said, I.M.; Aimi, N.; Takayama, H. Methyl chanofruticosinates from leaves of Kopsia flavida Blume. Phytochemistry 2001, 57, 603-606. [CrossRef]

113. Zheng, J.; Zhou, Y.; Huang, Z. The Isolation and Characterization of indole alkaloids from the fruits of Kopsia officinalis. Acta Chim. Sin. English Ed. 1989, 7, 168-175. [CrossRef]

114. Thomas, D.W.; Achenbach, H.; Biemann, K. Revised structures of the pleiocarpa alkaloids pleiocarpoline (pleiocarpine Nb-oxide), pleiocarpolinine (pleiocarpinine $\mathrm{Nb}$-oxide), and kopsinoline (kopsinine $\mathrm{Nb}$-oxide). J. Am. Chem. Soc. 1966, 88, 3423-3426. [CrossRef]

115. Kitajima, M.; Ohara, S.; Kogure, N. New Indole alkaloids from Melodinus henryi. heterocycles. Int. J. Rev. Commun. Heterocycl. Chem. 2012, 85, 1949-1959.

116. Burnell, R.H.; Chapelle, A.; Khalil, M.F. Quaternary bases from Hunteria eburnea pichon. Can. J. Chem. 1974, 52, 2327-2330. [CrossRef]

117. Wang, Z.; Shi, X.; Mu, Y.; Fang, L.; Chen, Y.; Lin, Y. Three novel indole alkaloids from Kopsia officinalis. Fitoterapia 2017,119, 8-11. [CrossRef]

118. Long, S.; Li, C.; Hu, J.; Zhao, Q.; Chen, D. Indole alkaloids from the aerial parts of Kopsia fruticosa and their cytotoxic, antimicrobial and antifungal activities. Fitoterapia 2018, 129, 145-149. [CrossRef] 
119. Kam, T.S.; Choo, Y.M. Kopsifolines A-F: A new structural class of monoterpenoid indole alkaloids from Kopsia. Helv. Chem. Acta 2004, 87, 991-998. [CrossRef]

120. Chen, X.D.; Hu, J.; Li, J.; Chi, F. Cytotoxic monoterpenoid indole alkaloids from the aerial part of Kopsia arborea. J. Asian Nat. Prod. Res. 2019, 22, 1024-1030. [CrossRef]

121. Liu, T.; Hu, J.; Li, J.; Chen, M. Cytotoxic monoterpenoid indole alkaloids from the aerial parts of Kopsia officinalis. J. Asian Nat. Prod. Res. 2020, 22, 724-731. [CrossRef]

122. Xie, T.; Zhao, Y.; He, J.; Zhao, L.; Wei, X.; Liu, Y.; Luo, X. Monoterpenoid indole alkaloids from the stems of Kopsia officinalis. Fitoterapia 2020, 104547. [CrossRef]

123. Bao, M.F.; Zeng, C.X.; Liu, Y.P.; Zhang, B.J.; Ni, L.; Luo, X.D.; Cai, X.H. Indole alkaloids from Hunteria zeylanica. J. Nat. Prod. 2017, 80, 790-797. [CrossRef] [PubMed]

124. Zhou, H.; He, H.P.; Kong, N.C.; Wang, Y.H.; Liu, X.D.; Hao, X.J. Three new indole alkaloids from the leaves of Kopsia officinalis. Helv. Chim. Acta 2006, 89, 515-519. [CrossRef]

125. Huang, Y.Z.; Huang, L.; Zhu, J.; Li, C.; Wu, G. Alkaloids of Kopsia officinalis. Acta Chim. Sin. 1984, 42, 82-83.

126. Yang, C.Q.; Ma, Y.F.; Chen, Y.G. Indole alkaloids from the twigs of Kopsia officinalis. Chem. Nat. Compd. 2017, 53, 595-597. [CrossRef]

127. Gan, C.Y.; Low, Y.Y.; Thomas, N.F.; Kam, T.S. Rhazinilam-Leuconolam-Leuconoxine alkaloids from Leuconotis griffithii. J. Nat. Prod. 2013, 76, 957-964. [CrossRef]

128. Cai, X.H.; Jiang, H.; Li, Y.; Cheng, G.G.; Liu, Y.P.; Feng, T.; Luo, X.D. Cytotoxic indole alkaloids from Melodinus fusiformis and M. morsei. Chin. J. Nat. Med. 2011, 9, 259-263. [CrossRef]

129. Jiang, H.; Li, Y.B.; Li, Y.; Li, L.; Ma, S.G.; Qu, J.; Yu, S.S. Analgesic corynanthe-type alkaloids from Strychnos angustiflora. Tetrahedron 2016, 72, 1276-1284. [CrossRef]

130. Robert, G.M.T.; Ahond, A.; Poupat, C.; Potier, P.; Jacquemin, H.; Kan, S.K. Aspidosperma de Guyane: Alcaloïdes des Graines de Aspidosperma oblongum. J. Nat. Prod. 1983, 46, 259-263. [CrossRef]

131. Gao, Y.; Yu, A.; Li, G.; Hai, P.; Li, Y.; Liu, J.; Wang, F. Hexacyclic monoterpenoid indole alkaloids from Rauvolfia verticillata. Fitoterapia 2015, 107, 44-48. [CrossRef]

132. Kumaria, R.; Rathib, B.; Ranic, A.; Bhatnagar, S. Rauvolfia serpentina L. Benth. ex Kurz.: Phytochemical, Pharmacological and Therapeutic Aspects. Int. J. Pharm. Sci. Rev. Res. 2013, 23, 348-355.

133. Kumar, S.; Singh, A.; Bajpai, V.; Srivastava, M.; Singh, B.P.; Ojha, S.; Kumar, B. Simultaneous Determination of Bioactive Monoterpene Indole Alkaloids in Ethanolic Extract of Seven Rauvolfia Species using UHPLC with Hybrid Triple Quadrupole Linear Ion Trap Mass Spectrometry. Phytochem. Anal. 2016, 296-303. [CrossRef] [PubMed]

134. Boğa, M.; Bingül, M.; Özkan, E.E.; Şahin, H. Chemical and Biological Perspectives of Monoterpene Indole Alkaloids from Rauwolfia species. Studies Nat. Prod. Chem. 2019, 61, 251-299.

135. Mahalakshmi, S.N.; Achala, H.G.; Ramyashree, K.R.; Kekuda, T.R. Rauvolfia tetraphylla L. (Apocynaceae) - A Comprehensive Review on Its Ethnobotanical Uses, Phytochemistry and Pharmacological Activities. Int. J. Pharm. Biol. Sci. $2019,9,664-682$.

136. Zeng, J.; Zhang, D.; Zhou, P.; Zhang, Q.; Zhao, L.; Chen, J.; Gao, K. Rauvomines A and B, Two Monoterpenoid Indole Alkaloids from Rauvolfia vomitoria. Org. Lett. 2017, 19, 3998-4001. [CrossRef]

137. Arthur, H.R.; Johns, S.R.; Lamberton, J.A.; Loo, S.N. Alkaloids of Rauwolfia verticillata (Lour.) Bail. of Hong Kong. Identification of vellosimine and peraksine, and demonstration from N.M.R. data that peraksine is a mixture of two epimers. Aust. J. Chem. 1968, 21, 1399-1401. [CrossRef]

138. Li, L.M.; Shi, S.D.; Liu, Y.; Zou, Q. Bioactivity-Guided Isolation and Identification of New and Immunosuppressive Monoterpenoid Indole Alkaloids from Rauvolfia yunnanensis Tsiang. Molecules 2019, 24, 4574. [CrossRef]

139. Zhang, Z.; ElSohly, H.N.; Jacob, M.R.; Pasco, D.S.; Walker, L.A.A.; Clark, A.M. New Indole Alkaloids from the Bark of Nauclea orientalis. J. Nat. Prod. 2001, 64, 1001-1005. [CrossRef]

140. Abaul, J.; Philogéne, E.; Bourgeois, P.; Mérault, G.; Poupat, C.; Ahond, A.; Potier, P. Alcaloïdes Indoliques De Rauvolfia Biauriculata. J. Nat. Prod. 1986, 49, 829-832. [CrossRef]

141. Schlittler, E.A.; Schwarz, H. Uber das Alkaloid Serpentin aus Rauwolfia serpentina Benth. Helv. Chim. Acta 1950, 33, 1463-1477. [CrossRef]

142. Geng, C.A.; Liu, X.K. Indole alkaloids from the leaves of Rauvolfia yunnanensis. Chem. J. Chin. U. 2010, 31, 731-735.

143. Staerk, D.; Lemmich, E.; Christensen, J.; Kharazmi, A.; Olsen, C.E.; Jaroszewski, J.W. Leishmanicidal, antiplasmodial and cytotoxic activity of indole alkaloids from Corynanthe pachyceras. Planta Med. 2000, 66, 531-536. [CrossRef] [PubMed]

144. Christiane, K.; Pierre, P.; Kan, S.K.; Jokela, R.; Lounasma, M. Indole alkaloids from Rauvolfia media. Phytochemistry 1986, 25, 1783-1784.

145. Wenkert, E.; Chang, C.J.; Chawla, H.P.S.; Cochran, D.W.; Hagaman, E.W.; King, J.C.; Orito, K. General Methods of Synthesis of Indole Alkaloids. 14. Short Routes of Construction of Yohimboid and Ajmalicinoid Alkaloid Systems and Their 13C Nuclear Magnetic Resonance Spectral Analysis. J. Am. Chem. Soc. 1976, 98, 3645-3655. [CrossRef]

146. Nunes, D.S.; Koike, L.; Taveira, J.J.; Reis, F.A.M. Indole alkaloids from Aspidosperma pruinosum. Phytochemistry 1992, 31, $2507-2511$.

147. Arbain, D.; Putra, D.P.; Sargent, M.V. The alkaloids of Ophiorrhiza filistipula. Aust. J. Chem. 1993, 46, 977-985. [CrossRef]

148. Reina, M.; Ruiz-Mesia, W.; Ruiz-Mesia, L.; Martínez-Díaz, R.; González-Coloma, A. Indole alkaloids from Aspidosperma rigidum and A. schultesii and their antiparasitic effects. Zeitschrift fur Naturforsch. Sect. C J. Biosci. 2011, 66, 225-234. 
149. Lin, M.; Yang, B.Q.; Yu, D.Q. Studies on the quaternary alkaloids of Rauvolfia verticillata (lour.) Baill var. Hainanensis Tsiang. Acta Pharm. Sin. 1986, 21, 114-118.

150. Sheludko, Y.; Gerasimenko, I.; Kolshorn, H.; Stöckigt, J. New alkaloids of the sarpagine group from Rauvolfia serpentina hairy root culture. J. Nat. Prod. 2002, 65, 1006-1010. [CrossRef]

151. Jin, Y.S.; Du, J.L.; Chen, H.S.; Jin, L.; Liang, S. A new indole alkaloid from Ervatamia yunnanensis. Fitoterapia 2010, 81, 63-65. [CrossRef]

152. Yu, D.Q.; Yang, J.S. Chemical Analytical Manual (the Second Edition), the Seventh Fascicle: NMR Spectra Analysis; Chemical Industry Press: Beijing, China, 2001; pp. 818-836.

153. Okuyama, E.; Gao, L.H.; Yamazaki, M. Analgesic Components from Bornean Medicinal Plants, Tabernaemontana pauciflora BLUME and Tabernaemontana pandacaqui POIR. Chem. Pharm. Bull. 1992, 40, 2075. [CrossRef]

154. Bartlett, M.F.; Dickel, D.F.; Taylor, W.I. The Alkaloids of Tabernanthe iboga. Part 1V. The Structures of Ibogamine, Ibogaine, Tabernanthine and Voacangine. J. Am. Chem. Soc. 1954, 80, 126-136.

155. Govindachari, T.R.; Joshi, B.S.; Saksena, A.K.; Sathe, S.S.; Viswanathan, N. The structure of heyneanine. Tetrahedron Lett. 1965, 43, 3873-3878. [CrossRef]

156. Thomas, D.W.; Biemann, K. The hydroxyindolenine derivative of voacangine, a new indole alkaloid from Voacanga africana. Tetrahedron 1968, 24, 4223-4231. [CrossRef]

157. Madinaveitia, A.; De La Fuente, G.; González, A. The Absolute Configuration at C(7) of Voacangine Hydroxyindolenine. Helv. Chim. Acta 1998, 81, 1645-1653. [CrossRef]

158. Das, B.C.; Fellion, E.; Plat, M. Alkaloids on Conopharyngia durissima seeds. Isolation of coronaridine, tabersonine, and coronaridine hydroxyindolenine. Comptes Rendus des Seances de l'Academie des Sciences, Serie D: Sciences Naturelles 1967, $264,1765-1767$.

159. Rastogi, K.; Kapil, R.S.; Popli, S.P. New alkaloids from Tabernaemontana divaricata. Phytochemistry 1980, 19, 1209-1212. [CrossRef]

160. Gunasekera, S.P.; Cordell, G.; Farnsworth, N.R. Anticancer indole alkaloids of Ervatamia heyneana. Phytochemistry 1980, 19, 1213-1218. [CrossRef]

161. Matos, F.J.A.; Fo, R.B.; Gottlieb, O.R.; Welbaneide, F.; Machado, L.; Iracema, M.; Madruga, L.M. 20-Epiheyneanine, an iboga alkaloid from Peschiera affinis. Phytochemistry 1976, 15, 551-553. [CrossRef]

162. Sharma, P.; Cordell, G.A. Heyneanine hydroxyindolenine, a new indole alkaloid from Ervatamia. J. Nat. Prod. 1988, 51, 528-531. [CrossRef]

163. Liang, S.; Chen, H.S.; Jin, Y.S.; Jin, L.; Lu, J.; Du, J.L. Study on chemical constituents in rhigome of Ervatamia hainanensis. J. Chin. Mater. Med. 2007, 32, 1296-1299.

164. Zhan, Z.J.; Yu, Q.; Wang, Z.L.; Shan, W.G. Indole alkaloids from Ervatamia hainanensis with potent acetylcholinesterase inhibition activities. Bioorganic Med. Chem. Lett. 2010, 20, 6185-6187. [CrossRef] [PubMed]

165. Guo, L.l.; Zhang, Y.; HE, H.P.; Li, Y.; Yu, J.P.; Hao, X.J. A new monoterpenoid indole alkaloid from Ervatamia chinensis. Chin. J. Nat. Med. 2012, 10, 226-229. [CrossRef]

166. Guoying, Z.; Hongping, H.; Bingui, W.; Xin, H.; Xiaojiang, H. A new indoloquinazoline alkaloid from the fruit of Evodia rutaecarpa. Acta Bot. Yunnan 2003, 25, 103-106.

167. Liu, Z.W.; Yang, T.T.; Wang, W.J.; Li, G.Q.; Tang, B.Q.; Zhang, Q.W.; Fan, C.L.; Zhang, D.M.; Zhang, X.Q.; Ye, W.C. Ervahainine A, a new cyano-substituted oxindole alkaloid from Ervatamia hainanensis. Tetrahedron Lett. 2013, 54, 6498-6500. [CrossRef]

168. Clivio, P.; Richard, B.; Deverre, J.R.; Sevenet, T.; Zeches, M.; Le Men-Oliver, L. Alkaloids from leaves and root bark of Ervatamia hirta. Phytochemistry 1991, 30, 3785-3792. [CrossRef]

169. Van Beek, T.A.; Verpoorte, R.; Svendsen, A.B.; Fokkens, R.J. Antimicrobially Active Alkaloids from Tabernaemontana chippii. J. Nat. Prod. 1985, 48, 400-423. [CrossRef]

170. Thomas, J.; Starmer, A. The isolation and identification of the major alkaloid present in Tabernaemontana pachysiphon stapf var cumminsi (stapf) H. Huber. J. Pharm. Pharmac. 1963, 15, 487. [CrossRef]

171. Pereira, P.S.; França, S.D.C.; Oliveira, P.V.A.D.; Breves, C.M.D.S.; Pereira, S.I.V.; Sampaio, S.V.; Nomizo, A.; Dias, D.A. Chemical Constituents from Tabernaemontana catharinensis Root Bark: A Brief NMR Review of Indole Alkaloids and in vitro Cytotoxicity. Quim. Nova 2008, 31, 20-24. [CrossRef]

172. Kam, T.; Sim, K. Five New Iboga Alkaloids from Tabernaemontana corymbosa. J. Nat. Prod. 2002, 65, 669-672. [CrossRef]

173. Low, Y.Y.; Lim, K.H.; Choo, Y.M.; Pang, H.S.; Etoh, T.; Hayashi, M.; Komiyama, K.; Kam, T.S. Structure, biological activity, and a biomimetic partial synthesis of the lirofolines, novel pentacylic indole alkaloids from Tabernaemontana. Tetrahedron Lett. 2010, 51, 269-272. [CrossRef]

174. Tang, B.Q.; Wang, W.J.; Huang, X.J.; Li, G.Q.; Wang, L.; Jiang, R.W.; Yang, T.T.; Shi, L.; Zhang, X.Q.; Ye, W.C. Iboga-type alkaloids from Ervatamia officinalis. J. Nat. Prod. 2014, 77, 1839-1846. [CrossRef] [PubMed]

175. Liu, Z.W.; Huang, X.J.; Xiao, H.L.; Liu, G.; Zhang, J.; Shi, L.; Jiang, R.W.; Zhang, X.Q.; Ye, W.C. New iboga-type alkaloids from Ervatamia hainanensis. RSC Adv. 2016, 6, 30277-30284. [CrossRef]

176. Azoug, M.; Loukaci, A.L.I.; Richard, B.; Nuzillard, J.; Moreti, C.; Zèches-Hanrot, M.; Le Men-Olivier, L. Alkaloids from stem bark and leaves of Peschiera buchtieni. Phytochemisty 1995, 39, 1223-1228. [CrossRef]

177. Rastogi, K.; Kapil, R.S.A.; Popli, S.P. Oxidation of Coronaridine. Heterocycles 1983, 20, 2001-2009.

178. De Souza, J.J.; Mathias, L.; Braz-Filho, R.; Vieira, I.J.C. Two new indole alkaloids from Tabernaemontana hystrix Steud. (Apocynaceae). Helv. Chim. Acta 2010, 93, 422-429. [CrossRef] 
179. Liu, Z.W.; Tang, B.Q.; Zhang, Q.H.; Wang, W.J.; Huang, X.J.; Zhang, J.; Shi, L.; Zhang, X.Q.; Ye, W.C. Ervaoffines E-G, three iboga-type alkaloids featuring ring C cleavage and rearrangement from Ervatamia officinalis. RSC Adv. 2017, 7, 21883-21889. [CrossRef]

180. Lim, K.H.; Raja, V.J.; Bradshaw, T.D.; Lim, S.H.; Low, Y.Y.; Kam, T.S. Ibogan, tacaman, and cytotoxic bisindole alkaloids from Tabernaemontana. Cononusine, an iboga alkaloid with unusual incorporation of a pyrrolidone moiety. J. Nat. Prod. 2015, 78, 1129-1138. [CrossRef]

181. Bartlett, M.F.; Dickel, D.F.; Maxfield, R.C.; Paszek, L.E.; Smith, A.F. The Alkaloids of Tabcrnanthe iboga. Part VIII. J. Am. Chem. Soc. 1959, 81, 1932-1935. [CrossRef]

182. Zhang, J.; Ao, Y.L.; Yao, N.; Bai, W.X.; Fan, C.L.; Ye, W.C.; Zhang, X.Q. Three new monoterpenoid indole alkaloids from Ervatamia pandacaqui. Chem. Biodivers. 2018, 15, 1800268. [CrossRef]

183. Chaturvedula, V.S.P.; Sprague, S.; Schilling, J.K.; Kingston, D.G.I. New Cytotoxic Indole Alkaloids from Tabernaemontana calcarea from the Madagascar Rainforest. J. Nat. Prod. 2003, 66, 528-531. [CrossRef]

184. Zhang, B.; Teng, X.; Bao, M.; Zhong, X.; Ni, L.; Cai, X. Cytotoxic indole alkaloids from Tabernaemontana officinalis. Phytochemistry 2015, 120, 46-52. [CrossRef] [PubMed]

185. Tan, S.; Low, Y.; Choo, Y.; Abdullah, Z.; Etoh, T.; Hayashi, M.; Komiyama, K.; Kam, T. Strychnan and Secoangustilobine A Type Alkaloids from Alstonia spatulata. Revision of the C-20 Configuration of Scholaricine. J. Nat. Prod. 2010, 73, 1891-1897. [PubMed]

186. Liu, Z.W.; Zhang, J.; Li, S.T.; Liu, M.Q.; Huang, X.J.; Ao, Y.L.; Fan, C.L.; Zhang, D.M.; Zhang, Q.W.; Ye, W.C.; et al. Ervadivamines A and B, Two Unusual Trimeric Monoterpenoid Indole Alkaloids from Ervatamia divaricata. J. Org. Chem. 2018, 83, 10613-10618. [CrossRef] [PubMed]

187. Zhang, H.; Wang, X.N.; Lin, L.P.; Ding, J.; Yue, J.M.J. Indole Alkaloids from Three Species of the Ervatamia Genus: E. officinalis, E.divaricata, and E. divaricata Gouyahua. J. Nat. Prod. 2007, 70, 54-59. [CrossRef] [PubMed]

188. Knox, J.R.; Slobbe, J. Indole alkaloids from Ervatamia orientalis. II. The constitution of the ervatamine group. Aust. J. Chem. 1975, 28, 1825-1841. [CrossRef]

189. Joule, J.A.; Monteiro, H.; Durham, L.J.; Gilbert, B.; Djerassi, C. Alkaloid studies. 48. The structure of apparicine, a novel aspidosperma alkaloid. Chem. Soc. (Resumed) 1965, 4773-4780. [CrossRef]

190. Watts, F.; Collera, O.; Sandoval, A. Alkaloids from Stemmadenia species. I. Alkaloids of S. donnellsmithii and S. galleottiana. Tetrahedron 1958, 2, 173-182.

191. Zhou, J.; Du, S.Y.; Dong, H.J.; Fang, L.; Feng, J.H. Preparative separation of monoterpenoid indole alkaloid epimers from ervatamia yunnanensis tsiang by ph-zone-refining counter-current chromatography combined with preparative high-performance liquid chromatography. Molecules 2019, 24, 1316. [CrossRef]

192. Athipornchai, A. A review on tabernaemontana spp.: Multipotential medicinal plant. Asian J. Pharm. Clin. Res. 2018, 11, 45-53. [CrossRef]

193. Nge, C.E.; Chong, K.W.; Thomas, N.F.; Lim, S.H.; Low, Y.Y.; Kam, T.S. Ibogan, Aspidosperman, Vincamine, and Bisindole Alkaloids from a Malayan Tabernaemontana corymbosa: Iboga Alkaloids with C-20 $\alpha$ Substitution. J. Nat. Prod. 2016, 79, 1388-1399. [CrossRef]

194. Kingston, D.G.; Gerhart, B.B.; Ionescu, F.; Mangino, M.M.; Sami, S.M. Plant Anticancer Agents V: New Bisindole Alkaloids from Tabernaemontana johnstonii Stem Bark. Pharm. Sci. 1978, 67, 249-251. [CrossRef] [PubMed]

195. Kam, T.S.; Sim, K.M. New Tabernamine Derivatives from Tabernaemontana. Heterocycles 2002, 57, 2137-2143. [CrossRef]

196. Qu, Y.; Simonescu, R.; De Luca, V. Monoterpene Indole Alkaloids from the Fruit of Tabernaemontana litoralis and Differential Alkaloid Composition in Various Fruit Components. J. Nat. Prod. 2016, 79, 3143-3147. [CrossRef] [PubMed]

197. Yuwen, H.; Yuan, Y.; Hao, X.; He, H.; Zhang, Y. Two new monoterpenoid indole alkaloids from Tabernaemontana divaricata. Nat. Prod. Res. 2019, 33, 2139-2144. [CrossRef] [PubMed]

198. Sim, D.S.Y.; Chong, K.W.; Nge, C.E.; Low, Y.Y.; Sim, K.S.; Kam, T.S. Cytotoxic Vobasine, Tacaman, and Corynanthe-Tryptamine Bisindole Alkaloids from Tabernaemontana and Structure Revision of Tronoharine. J. Nat. Prod. 2014, 77, 2504-2512. [CrossRef] [PubMed]

199. Zèches-Hanrot, M.; Nuzillard, J.M.; Richard, B.; Schaller, H.; Hadi, H.A.; Sévenet, T.; Le Men-Olivier, L. Alkaloids from leaves and stem bark of Ervatamia peduncularis. Phytochemistry 1995, 40, 587-591. [CrossRef]

200. Clivio, P.; Guillaume, D.; Vercauteren, J.; Richard, B.; Nuzillard, J.M.; Zèches-Hanrot, M.; Le Men-Olivier, L. Two bis-indole alkaloids from leaves of Ervatamia polyneura. Phytochemistry 1995, 40, 953-959. [CrossRef]

201. Cai, Y.; Sarotti, A.M.; Zhou, T.; Huang, R.; Qiu, G.; Tian, C.; Cao, S.; Mándi, A.; Kurtán, T.; Yang, S.; et al. Flabellipparicine, a Flabelliformide-Apparicine-Type Bisindole Alkaloid from Tabernaemontana divaricata. Nat. Prod. 2018, 81, 1976-1983. [CrossRef]

202. Feng, X.Z.; Kan, C.; Husson, H.P.; Potier, P.; Kan, S.K.; Lounasmaa, M. Nouveaux alcaloïdes indoliques dimeres de type voacamine extraits d'Ervatamia hainanensis. J. Nat. Prod. 1981, 44, 670-675. [CrossRef]

203. Van Beek, T.A.; Verpoorte, R.; Svendsen, A.B. Isolation and synthesis of vobparicine, a novel type dimeric indole alkaloid. Tetrahedron Lett. 1984, 25, 2057-2060. [CrossRef]

204. Ingkaninan, K.; Changwijit, K.; Suwanborirux, K. Vobasinyl-iboga bisindole alkaloids, potent acetylcholinesterase inhibitors from Tabernaemontana divaricata root. J. Pharm. Pharmacol. 2006, 58, 847-852. [CrossRef] [PubMed]

205. Henriques, A.T.; Melo, A.A.; Moreno, P.R.H.; Ene, L.L.; Henriques, J.A.P.; Schapoval, E.E.S. Ervatamia coronaria: Chemical constituents and some pharmacological activities. J. Ethnopharmacol. 1996, 50, 19-25. [CrossRef] 
206. Yamauchi, T.; Abe, F.; Padolina, W.G.; Dayrit, F.M. Alkaloids from leaves and bark of Alstonia scholaris in the Philippines. Phytochemistry 1990, 29, 3321-3325. [CrossRef]

207. Xu, J.; Qu, W.; Cao, W.Y.; Wang, Y.; Zheng, K.J.; Luo, S.Z.; Wu, M.-Y.; Liu, W.-Y.; Feng, F.; Zhang, J. Chemical Constituents from Tabernaemontana bufalina Lour. Chem. Biodivers. 2019, 16, 1800491. [CrossRef] [PubMed]

208. Yu, Y.; Gao, J.M.; Liu, J.K. Two New Oxindole Alkaloids from Ervatamia yunnanensis. Chin. Chem. Lett. 1999, 10, 575-578.

209. Perera, P.; Samuelsson, G.; van Beek, T.A.; Verpoorte, R. Tertiary Indole Alkaloids from Fruits of Tabernaemontana dichotoma. Planta Med. 1984, 50, 148-150. [CrossRef]

210. Melacheu, G.L.F.; Njoya, E.M.; Jouda, J.B.; Kweka, B.N.W.; Mbazoa, C.D.; Wang, F.; Wandji, J. Two new indole alkaloids from Tabernaemontana contorta Stapf. Phytochem. Lett. 2019, 30, 116-119. [CrossRef]

211. Yu, Y.; Bao, M.F.; Wu, J.; Chen, J.; Yang, Y.R.; Schinnerl, J.; Cai, X.H. Tabernabovines A-C: Three Monoterpenoid Indole Alkaloids from the Leaves of Tabernaemontana bovina. Org. Lett. 2019, 21, 5938-5942. [CrossRef]

212. Chatterjee, A.; Banerji, A.; Majumder, P.; Majumder, R. Alkaloids of Rhazya stricta Decaisne Studies on Rhazinaline and Geissoschizine. Bull. Chem. Soc. Jpn. 1976, 49, 2000-2004. [CrossRef]

213. Mukhopadhyay, S.; Handy, G.A.; Funayama, S.; Cordell, G.A. Anticancer indole alkaloids of Rhazya stricta. J. Nat. Prod. 1981, 44, 696-700. [CrossRef]

214. Baeshen, M.N.; Khan, R. Therapeutic Potential of the Folkloric Medicinal Plant Rhazya stricta. Biol. Syst. 2015, 5, 1-5. [CrossRef]

215. Ahmed, A.; Li, W.; Chen, F.; Zhang, J.; Tang, Y.; Chen, L.; Tang, G. Monoterpene indole alkaloids from Rhazya stricta. Fitoterapia 2018, 128, 1-6. [CrossRef] [PubMed]

216. Gelling, A.; Jeffery, J.C.; Poveycand, C.; Went, M.J.; Chemistry, I.; Its, B.B.S. The Isolation of Monomeric Secodine-type Alkaloids from Rhazya Species. Chem. Sci. 1991, 346, 349-351.

217. Kan, C.; Deverre, J.R.; Sevenet, T.; Quirion, J.C.; Husson, H.P. Indole Alkaloids from Kopsia Deverrei. Nat. Prod. Lett. 1995, 7, 275-281. [CrossRef]

218. Khanum, S. Strictamine-N-oxide from Rhazya stricta. Phytochemistry 1984, 23, 709-710.

219. Abe, F.; Yamauchi, T. Indole alkaloids from leaves and stems of Leuconotis eugenifolius. Phytochemistry 1993, 35, 169-171. [CrossRef]

220. Proksa, B.; Uhrín, D.; Grossmann, E.; Votický, Z. New Quaternary Alkaloids from Vinca minor. Planta Med. 1989, 55, 188-190. [CrossRef]

221. Lounasmaa, M.; Hanhinen, P. Conformational study of geissoschizine isomers and their model compounds. Heterocycles 1999, 51, 649-670. [CrossRef]

222. Kutney, J.P.; Brown, R.T. The structural elucidation of sitsirikine, dihydrositsirikine and isositsirikine: Three new alkaloids from vinca rosea linn. Tetrahedron 1966, 22, 321-336. [CrossRef]

223. Danieli, B.; Lesma, G.; Palmisano, G.; Riva, R.; Tollari, S. Absolute Configuration of (-)-Vincatine, the Unique 2,16-Seco Aspidosperma Alkaloid. J. Org. Chem. 1984, 547-551. [CrossRef]

224. Jewers, K.; Pusey, D.F.G.; Sharma, S.R.; Ahmad, Y. ${ }^{13}$ C-NMR Spectral Analysis of Rhazine, Quebrachamine and Rhazinilam. Planta Med. 1980, 38, 359-362. [CrossRef]

225. Atta-ur-Rahman; Habib-ur-Rehman; Ahmad, Y.; Fatima, K.; Badar, Y. Studies on the Alkaloids of Rhazya stricta. Planta Med. 1987, 53, 256-259. [CrossRef] [PubMed]

226. Abdul-Hameed, Z.H.; Alarif, W.M.; Omer, A.; El Omri, A.; Ayyad, S.-E.N.; Badria, F.A.; Neamatallah, T.; Bawakid, N.O. Selective Anti-proliferative Activity of Indole Alkaloids from Rhazya stricta Decne Leaves. Lett. Org. Chem. 2019, 16, 941-947. [CrossRef]

227. Hajrah, N.H.; Abdul, W.M.; Abdul-hameed, Z.H.; Alarif, W.M.; Al-abbas, N.S.A.; Ayyad, S.E.N.; Omer, A.M.S.; Mutawakil, M.Z.; Hall, N.; Obaid, A.Y.; et al. Gene Expression Profiling to Delineate the Anticancer Potential of a New Alkaloid Isopicrinine From Rhazya stricta. Integr. Cancer Ther. 2020, 19, 1-13. [CrossRef]

228. Abdul-Hameed, Z.H.; Alarif, W.M.; Sobhi, T.R.; Abdel-Lateff, A.; Ayyad, S.E.N.; Badria, F.A.; Saber, J. New cytotoxic indole-type alkaloids obtained from Rhazya stricta leaves. South. Afr. J. Botany 2021, 137, 298-302. [CrossRef]

229. Dewick, P.M. Medicinal Natural Products; a Biosynthetic Approach, 3rd ed.; John Wiley \& Sons: Hoboken, NJ, USA, 2009.

230. O'Connor, S.E.; Maresh, J.J. Chemistry and biology of monoterpene indole alkaloid biosynthesis. Nat. Prod. Rep. 2006, $23,532-547$. [CrossRef]

231. Bi, Y.; Hamaker, L.K.; Cook, J.M. The Synthesis of Macroline Related Alkaloids. Stud. Nat. Pro. Chem. 1993, 13, $383-432$.

232. Esmond, R.W.; LeQuesne, P.W. Biomimitic synthesis of macroline. J. Am. Chem. Soc. 1980, 102, 7116-7117. [CrossRef]

233. Namjoshi, O.A.; Cook, J.M. Sarpagine and related-alkaloids. Alkaloids Chem. Biol. 2016, 76, 63-169. 